\title{
Real-Time Image Based \\ Lighting for 360 Degree \\ Panoramic Video
}

by

Thomas Iorns

\author{
A thesis \\ submitted to the Victoria University of Wellington \\ in partial fulfilment of the \\ requirements for the degree of \\ Master of Science \\ in Computer Science.
}

Victoria University of Wellington

2016 



\begin{abstract}
The application of the newly popular content medium of 360 degree panoramic video to the widely used offline lighting technique of image based lighting is explored, and a system solution for real-time image based lighting of virtual objects using only the provided 360 degree video for lighting is developed. The system solution is suitable for use on live streaming video input, and is shown to run on consumer grade graphics hardware at the high resolutions and framerates necessary for comfortable viewing on head mounted displays, rendering at over 60 frames per second for stereo output at $1182 \times 1464$ per eye on a mid-range graphics card. Its use in several real-world applications is also studied, and extension to consider real-time shadowing and reflection is explored.
\end{abstract}




\section{Acknowledgments}

Thanks to my supervisor Taehyun Rhee for all his support and advice, and my colleagues Andrew Chalmers, Kieran Carnegie and Ben Allen for their valuable input at various stages of production.

Work for this thesis was carried out with the support of New Zealand's Ministry of Business, Innovation and Employment (MBIE), under the Human-Digital Content Interaction for Immersive 4D Home Entertainment ( $\left.\mathrm{HDI}^{2} 4 \mathrm{D}\right)$ project.

Parts of this thesis include work done in collaboration with Kiran Nassim (Ewha Womans University), Joshua Chen (HIT Lab NZ / University of Canterbury) and Jaedong Lee (Korea University) for the $\mathrm{HDI}^{2} 4 \mathrm{D}$ project, and Kurt Ma and Andrew Chalmers (Victoria University of Wellington) for independent publication. 


\section{List of Publications}

- Thomas Iorns, Taehyun Rhee, "Real-Time Image Based Lighting for 360 Degree Panoramic Video", Lecture Note in Computer Science, Springer, Presented in PSIVT workshop, Vision Meets Graphics 2015, Auckland, NZ, Nov, 2015.

- Wan Duo Ma, Thomas Iorns, Andrew Chalmers, and Taehyun Rhee, "Synthesizing Radiance Maps from Legacy Outdoor Photographs for Real-time IBL on HMDs", Proc. of 30th International Conference on Image and Vision Computing New Zealand (IVCNZ 2015), Auckland, NZ, Nov, 2015. 


\section{Contents}

1 Introduction 1

2 Background $\quad 3$

2.1 High Dynamic Range (HDR) Images . . . . . . . . . . . . . . 3

2.2 Image Based Lighting (IBL) . . . . . . . . . . . . . . 6

2.3 Virtual Reality (VR), Augmented Reality (AR), Mixed Reality (MR), and Head Mounted Displays (HMDs) . . . . . . . . . . 10

2.4360 Degree Panoramic Video (360 video) . . . . . . . . . . . . . 10

2.5 Real-Time Image Based Lighting Techniques . . . . . . . . . . . 12

2.6 Reduced Resolution Equivalence . . . . . . . . . . . . . . 15

2.7 LDR-HDR Tonemapping . . . . . . . . . . . . . . . 16

3 Prior Work 19

3.1 Adding Virtual Objects to Real Scenes using IBL . . . . . . . . . . 19

3.2 Real-Time IBL . . . . . . . . . . . . . . . . 20

3.3 Real-Time IBL using Spherical Harmonics . . . . . . . . . . . . . 21

3.4 Real-Time IBL using HDR video . . . . . . . . . . . . . . 22

3.5 Real-Time IBL using Omnidirectional Video . . . . . . . . . . . 24

3.6 Filtered Importance Sampling . . . . . . . . . . . . . . . 25

4 System Solution $\quad 29$

4.1 Basic Real-Time Image Based Lighting . . . . . . . . . . . . . . . 29

4.1 .1 Problem Description . . . . . . . . . . . . . . . 30 
4.1.2 Basic System Solution . . . . . . . . . . . . . . . 30

4.1.3 Prototype Implementation . . . . . . . . . . . . . . 32

4.1 .4 Results . . . . . . . . . . . . . . . 36

4.2 Real-Time IBL for 360 Degree Panoramic Video . . . . . . . . . . 40

4.2.1 Problem Description . . . . . . . . . . . . . . 40

4.2.2 System Solution . . . . . . . . . . . . . . . 40

4.2.3 Prototype Implementation . . . . . . . . . . . . . 41

4.2 .4 Results . . . . . . . . . . . . . . . 45

5 Applications $\quad 53$

5.1 Implementation in Unity3D . . . . . . . . . . . . . . . 53

5.1 .1 Technical Challenges . . . . . . . . . . . . 54

5.1 .2 Implementation . . . . . . . . . . . . . 54

5.1 .3 Results . . . . . . . . . . . . . . 58

5.2 Interactive 4D Home Entertainment System Demo . . . . . . . . . 58

5.2.1 Technical Challenges . . . . . . . . . . . . . . 59

5.2 .2 Implementation . . . . . . . . . . . . . . . 59

5.2 .3 Results . . . . . . . . . . . . . . 60

5.2 .4 Acknowledgements . . . . . . . . . . . . . 63

5.3 IBL using Non-Panoramic Legacy Photographs . . . . . . . . . . 63

5.3.1 Technical Challenges . . . . . . . . . . . . 65

5.3 .2 Implementation . . . . . . . . . . . 65

6 Self-Shadowing and Self-Reflection 69

6.1 Problem Description . . . . . . . . . . . . . . . . . . . 69

6.2 Background ..................... 70

6.2 .1 Self-Shadowing . . . . . . . . . . . 70

6.2.2 Coherent Shadow Maps . . . . . . . . . . . . . . . . 71

6.2.3 Layered Depth Images . . . . . . . . . . . . . . 73

6.3 Orthographic Linearized Layered Fragment Buffers (OLLFBs) . . . 76 6.3 .1 Implementation . . . . . . . . . . . . 76

6.4 Results . . . . . . . . . . . . . . . . . . 77 
7 Conclusion 


\section{Chapter 1}

\section{Introduction}

Image Based Lighting (IBL) [10] has long since become a staple lighting technique for physically accurate rendering of virtual objects and scenes. For over a decade it has been widely adopted by the film industry as a tool for rendering computer generated objects in such a way that they can believably be merged with real footage. The realism of the technique is such that it has been shown to be able to render virtual objects so as to be indistinguishable from real photographed objects [9], however this realism comes with the drawback of requiring a large amount of time to render an image. For high quality ray traced results, times of minutes to hours are not unheard of for the rendering of a single video frame.

This has led to various attempts to reduce the computation cost by simplifying the lighting distribution [41], computing parts of the calculation in advance [16], or by coming up with efficient approximations to or simplifications of the ray tracing algorithm [1]. These have various drawbacks, primarily requirements that only certain material types be used, or that the environment image used for lighting be analyzed in advance. The benefit is that once one of these assumptions is made the basic IBL calculations can be easily run in real-time on modern graphics hardware.

Unfortunately even when applying these more efficient methods, the real-time use of IBL is still held back by the need for a panoramic image of the environment in a certain format (see section 2.1), which cannot easily be obtained without specialized skills and knowledge. Other than in entirely virtual environments (such as in 
3D games, which do commonly make good use of IBL), the necessary scene capture is unlikely to be available unless detailed preparation is made.

The recently emerging popularity of two special-purpose pieces of hardware however, provides us with an interesting potential source of panoramic environment images, that is 360 degree panoramic video (360 video). The application of this suddenly widely-available content medium to the field of real-time image based lighting will be the primary focus of this thesis.

The popularity of 360 video, which is now widely shared on websites such as YouTube [54], has been driven by the rising popularity of consumer Head Mounted Displays (HMDs) such as the Oculus Rift [51]. These displays allow one to turn one's head and look around an entire scene, and making good advantage of this 360 video has proven a cheaply-obtainable yet effective form of entertainment for use on these devices. Various special purpose video cameras have become available for capturing 360 video, as have much cheaper mounts which enable capture using an arrangement of standard digital video cameras such as the popular GoPro [19].

If 360 degree panoramic video could be easily used for image based lighting, it could potentially extend the usefulness and entertainment value of this medium, and perhaps enable fully believable augmented or mixed reality environments based on live 360 degree video.

In this thesis the barriers to such an application are examined, and a system solution allowing real-time image based lighting to be performed using live 360 degree panoramic video input is developed. The system is shown to be capable of running on consumer-grade graphics hardware and rendering to HMD at the high resolutions and framerates required for an immersive and comfortable experience. Potential real-world applications of the technology are then examined, and the system is found to perform well when applied. The possibility for further extension of the system is also considered, and a potential solution proposed for real-time shadows and reflections in addition to lighting. 


\section{Chapter 2}

\section{Background}

Certain terms and background are important for comprehension of the rest of this text, and will be referred to frequently. For convenience these are discussed before the body of the work. Acronyms introduced here will be commonly used, and thus some level of familiarity with this material is recommended.

\section{High Dynamic Range (HDR) Images}

Standard digital images have a limited dynamic range, which is to say that the bright colours may only be so bright, and the dim colours only so dim. A typical image such as a digital photograph will have brightness values in the discrete integer range between 0 and 255, with a value of 255 representing the brightest colour that can be displayed and a value of 0 representing the darkest. Thus for an image with only shades of grey, 0 would represent pure black and 255 pure white with 1 representing the darkest shade of grey that can be reproduced and 254 representing the lightest.

This is a useful format for representing images which may be printed onto passive media such as paper, as in this case the brightest value is limited by the intensity of the ambient light shining onto the paper. This maximum value cannot be exceeded by the printed image, and its achievement is desirable for vivid colour reproduction. Standard computer displays also mimic this behaviour, with their maximum brightness being set to an intensity which is comfortable for viewing. This maximum 


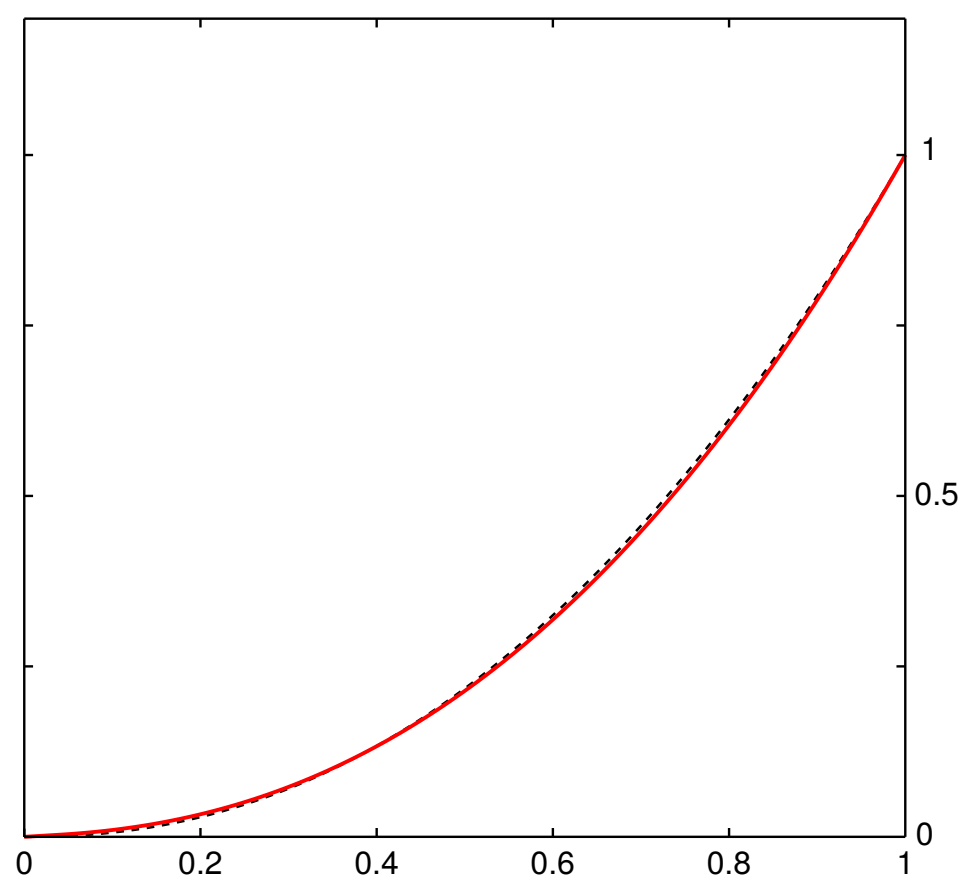

Figure 2.1: sRGB gamma curve (red line) compared with straight 2.2 exponent (dashed black line). Horizontal axis represents input intensity, and vertical axis output intensity. Image adapted from https://commons.wikimedia.org/wiki/ File:SRGB_gamma.svg

intensity is treated as paper-white.

The meaning of the values between 0 and 255 is not typically linear in intensity as measured on a physical scale (see figure 2.1), but instead conforms to a representation closer to what humans perceive as they look at a scene in real life. Most digital colour images viewed today use the sRGB [4] profile depicted in figure 2.1, and this is sufficient for perceptually accurate rendition of real life scenes. Using this colour profile, a maximum dynamic range of around 3000:1 is represented, with the value of 255 representing approximately 3000 times the intensity of a value of 1 .

While this is sufficient to represent an image that appears perceptually accurate to human eyes, it does not necessarily capture all of the important detail in a scene. If there are two areas of highly differing brightness within a scene, it can be difficult to accurately portray using only the values of $0-255$. An example is that of an unlit 

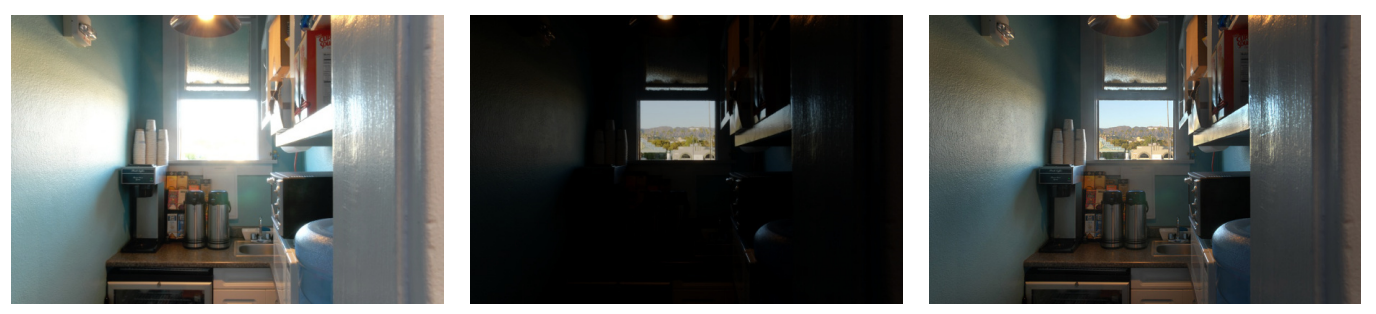

Figure 2.2: Example demonstrating the need for HDR images. Representing the interior faithfully leaves the exterior white, and representing the exterior faithfully leaves the interior black. An image combining the two more accurately represents how the scene appears to a human viewer, and may also preserve information about the relative brightness of the interior and exterior if stored in an HDR format. Images from the interactive viewer at http://www.hdrlabs.com/gallery/realhdr/.

room with a window looking out onto a sunny outdoor scene (see figure 2.2). If we set the maximum brightness such that the details of the interior are visible, the window will appear completely white, as all of the outdoor scene exceeds the maximum portrayable brightness. If we set it such that the exterior scene is visible, the interior would appear completely black, as the interior brightness does not achieve the minimum value. In real life we can see both scenes as our eyes adjust as we look from one area to the other, but this cannot be represented accurately using a single image of standard dynamic range.

What we can do in this case is capture two images, one which captures the detail of the bright part, and one which captures the detail of the dim part. These can then be merged into a High Dynamic Range (HDR) image, which will use more values than 255 to represent the brightness of a pixel in the image. The commonly used OpenEXR [7] image format typically stores values in 16-bit floating point format, resulting in a maximum dynamic range of around a billion to one. With such dynamic range, values will usually be stored linearly proportional to physical intensity, as this makes the image more convenient to process.

By taking photographs with multiple different exposure settings, HDR images can be obtained that represent real-life scenes accurately, even for extreme cases such as when the camera is pointing directly at a bright light source such as the sun. 
When dealing with HDR images, standard images are usually referred to as Low Dynamic Range (LDR). Various potential mappings exist for converting one to the other, and this will be discussed further on in the thesis.

\section{Image Based Lighting (IBL)}

An application for which HDR images are usually required is Image Based Lighting (IBL), which uses a full spherical panoramic image (such as that of figure 2.4c) to light virtual objects realistically. IBL was described early on by Miller and Hoffman [35] but was popularized by Debevec [10] who has, among other applications, used it to convincingly render virtual objects into real-life photographs [11].

So as to have an accurate description of the intensity of incoming light, an HDR image with values linearly proportional to intensity is ideal for IBL. In this form, the image can be interpreted as an irradiance map describing the amount of incident light according to direction, which can be used to numerically solve the rendering equation

$$
L_{o}\left(\omega_{o}\right)=\int_{\Omega} L_{i}\left(\omega_{i}\right) f_{r}\left(\omega_{o}, \omega_{i}\right)\left(\omega_{i} \cdot \vec{n}\right) d \omega_{i}
$$

at discrete points on the surface of the virtual object, directly approximating the colour of light reflected from these point to the viewer's eye. Here $L_{o}\left(\omega_{o}\right)$ is the output luminosity at angle $\omega_{o}, L_{i}\left(\omega_{i}\right)$ is the incident luminosity from angle $\omega_{i}$, $f_{r}\left(\omega_{o}, \omega_{i}\right)$ is a reflectance function describing the amount of light reflected from direction $\omega_{i}$ to direction $\omega_{o}$ for a given material, $\vec{n}$ is the surface normal direction, and $\Omega$ is the solid angle of the hemisphere around the surface normal.

Along with traditional rasterizing techniques this can lead to a very accurate depiction of the virtual object, so much so that they can be indistinguishable from real objects, and the mentioned application of this technique by Debevec [11] convincingly inserts virtual objects into real-life photographs with great accuracy (see figure 2.3). For this however there are two primary drawbacks.

The first drawback to traditional IBL is that it is computationally expensive. The 


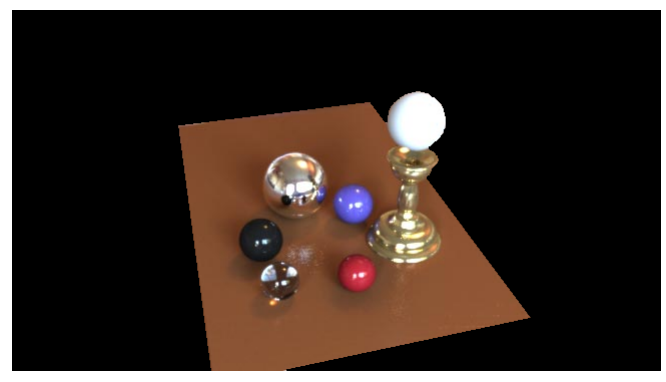

(a) Scene with virtual objects.

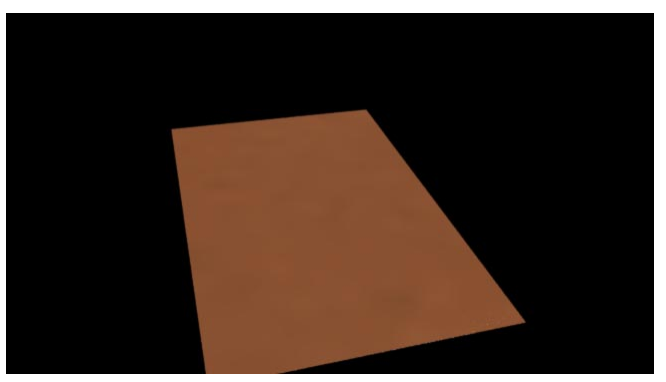

(b) Scene without virtual objects.

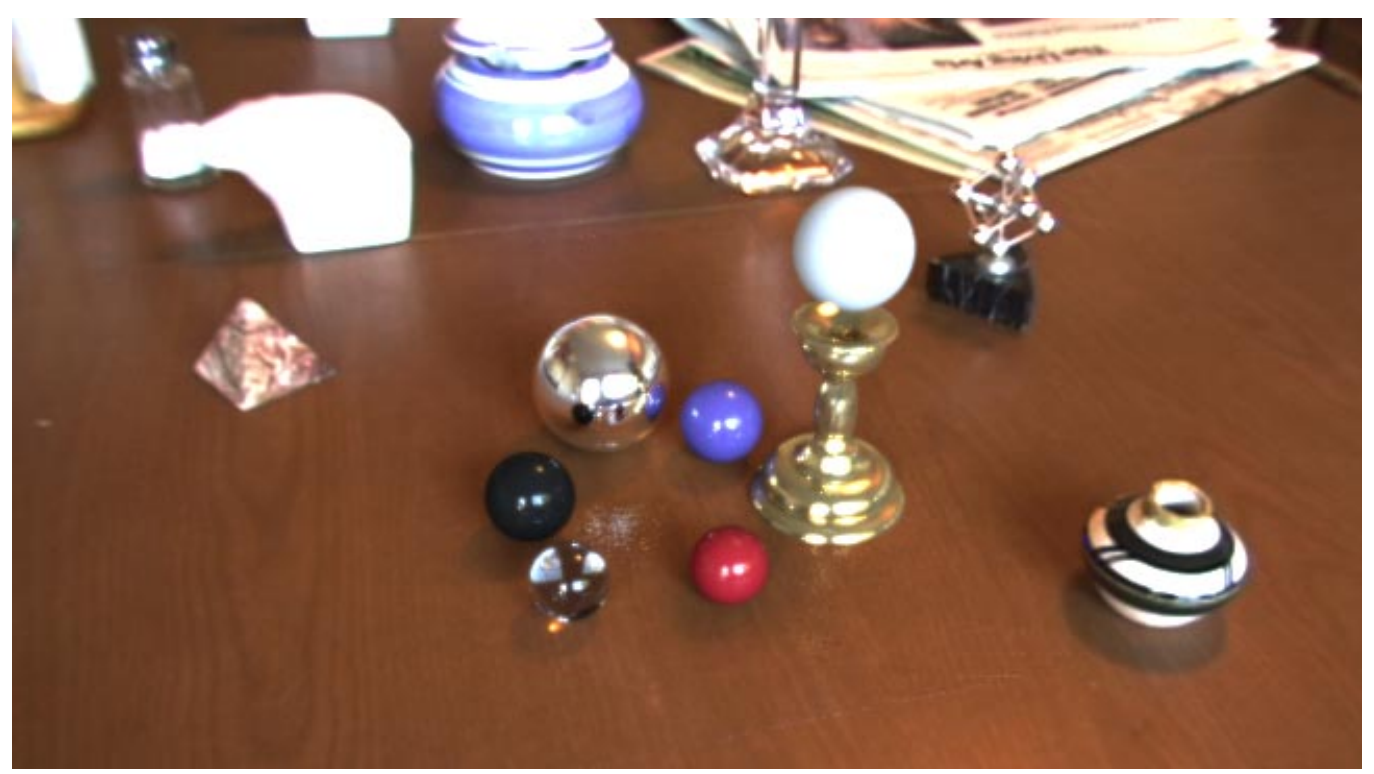

(c) Final scene with virtual objects added to original photograph. Shadows from the virtual plane are transferred onto the table in the photograph. The prominent horizontal line near the top of the output is in fact part of the original photograph.

Figure 2.3: Differential rendering using IBL. Treatment here is simplified and not the full procedure. Images are from [11]. 
rendering equation requires that we integrate over an entire hemisphere for every rendered point on the object, so if the object takes up an $A$ by $B$ area in the rendered image, and the irradiance map has a resolution of $C$ by $D$, computation time is proportional to $A B C D$, essentially an $\mathrm{O}\left(N^{4}\right)$ operation. As such IBL is traditionally an offline technique, requiring minutes or hours to render a single frame, but with very realistic results.

The second drawback to IBL is that it requires one to posess or acquire a full HDR spherical panorama of the environment at precisely the position where the virtual object will appear. For fully constructed 3D scenes this is not a problem, and for many applications it is sufficient to use a previously captured HDR panorama of a sky and distant landscape. However if we want to match the lighting of virtual objects with a real-life scene such as in [11], this requires special preparation.

In the case of [11], two images of the scene are captured. One image is the desired final image, but without whatever virtual objects are to be inserted. The other is an HDR image with a shiny metal ball (called a light probe) placed at the position where the virtual object will be inserted. This light probe reflects the environment, so from its image the environment can be determined. The probe is treated as a perfectly reflective sphere, and an irradiance map is reconstructed from the reflection on its surface. This irradiance map is then used to accurately light the virtual object which is rendered and inserted into the scene.

Because the mapping from light probe image to environment does not distribute detail evenly, and because finding a shiny metal ball is not always convenient, these days panoramic HDR environment images are usually obtained using a digital camera and fisheye lens. From a point near where the object will be, fisheye photos are taken at multiple exposures and in multiple directions. These photos are then combined using special-purpose software (such as [40]), to result in a single HDR spherical panorama.

Typically the resulting panorama will be in either latlong (figure 2.4c) or cubemap (figure 2.4d) format. Latlong images easily translate to spherical coordinates, with horizontal image position corresponding directly to longitude (or azimuth), and vertical image position corresponding directly to latitude (or elevation), so they are 


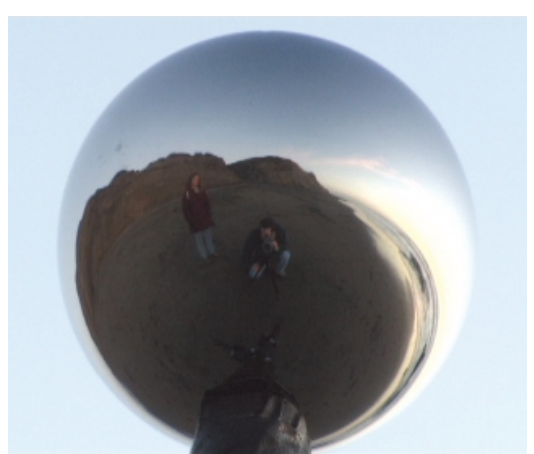

(a) Light probe. A standard photograph of a shiny metal ball. Image from [10].

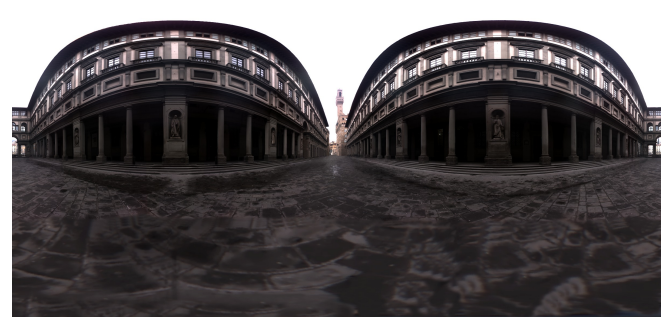

(c) Latitude-Longitude (lat-long or latlong) format environment image. Horizontal position corresponds to azimuthal angle and vertical position to elevation angle on a spherical projection.

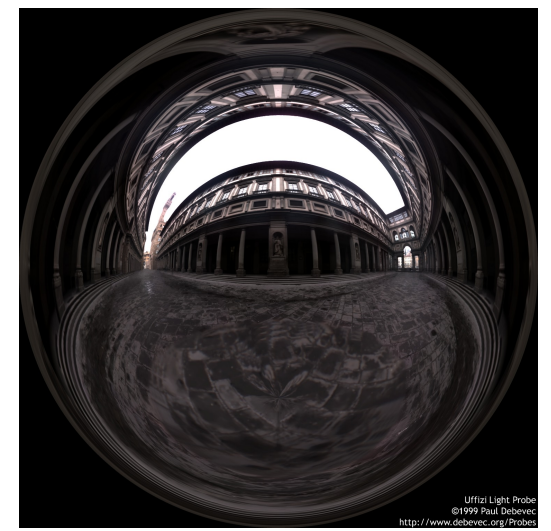

(b) Angular map format environment image. Distance from center corresponds to angular distance from a point in the environment. This can be formed from one or more light probes.

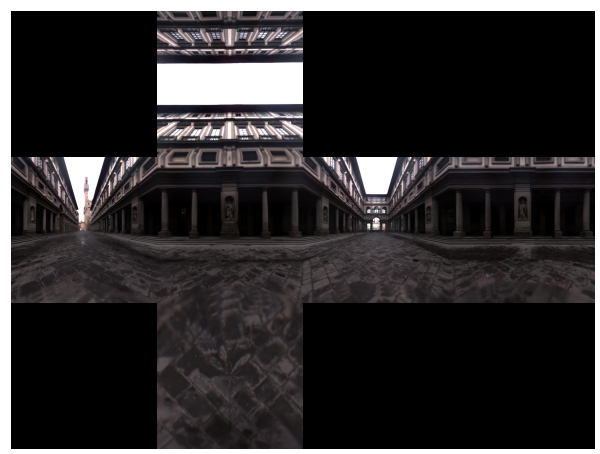

(d) Cubemap format environment image. Each square region corresponds to a perspective projection along the $\mathrm{X}, \mathrm{Y}$ or $\mathrm{Z}$ axis with 90 degree field of view. The combined result can be thought of as the environment projected onto a cube.

Figure 2.4: Environment images in several formats. With the exception of the light probe, the rest are of the same environment: a road between buildings on an overcast day. Unless otherwise mentioned, images are from http://www. pauldebevec. com/Probes/. 
easy to work with. Cubemaps have less distortion and are commonly supported by dedicated graphics hardware, but must be dealt with piecewise so are less convenient when calculations are programmed manually.

\section{Virtual Reality (VR), Augmented Reality (AR), Mixed Reality (MR), and Head Mounted Displays (HMDs)}

Over the course of computing (and science fiction) history there has been enough interest in Virtual Reality (VR) that it has become a commonly used term in general language. Augmented Reality (AR) has also had surges in popularity, although consumer devices have yet to take off. VR refers to the experience of immersion in a completely virtual environment, as if it were reality. AR refers to the addition of virtual elements into a predominantly real-life environment. Mixed Reality (MR) refers to any mixture of real and virtual elements, and can have a fairly broad interpretation.

For VR an entirely virtual environment is desired, so a common approach is to use an enclosed Head Mounted Display (HMD) (see figure 2.5). The HMD will typically display two separate images on a screen strapped to the participant's face, one image for each eye, allowing for controlled visual perception (including depth perception) across a fairly wide field of view. If a virtual environment is constructed and displayed appropriately, the viewer can perceive it as a believable virtual space they are immersed in.

\section{Degree Panoramic Video (360 video)}

Recently thanks to reductions in price, consumer level HMDs such as the Oculus Rift [51] (figure 2.5) have become increasingly popular, stimulating demand for entertainment content aimed at these devices. One popular form of content which has emerged is known as 360 Degree Panoramic Video (360 degree video, 360 video, 360 video), video which captures most of, or the entirety of a full spherical 

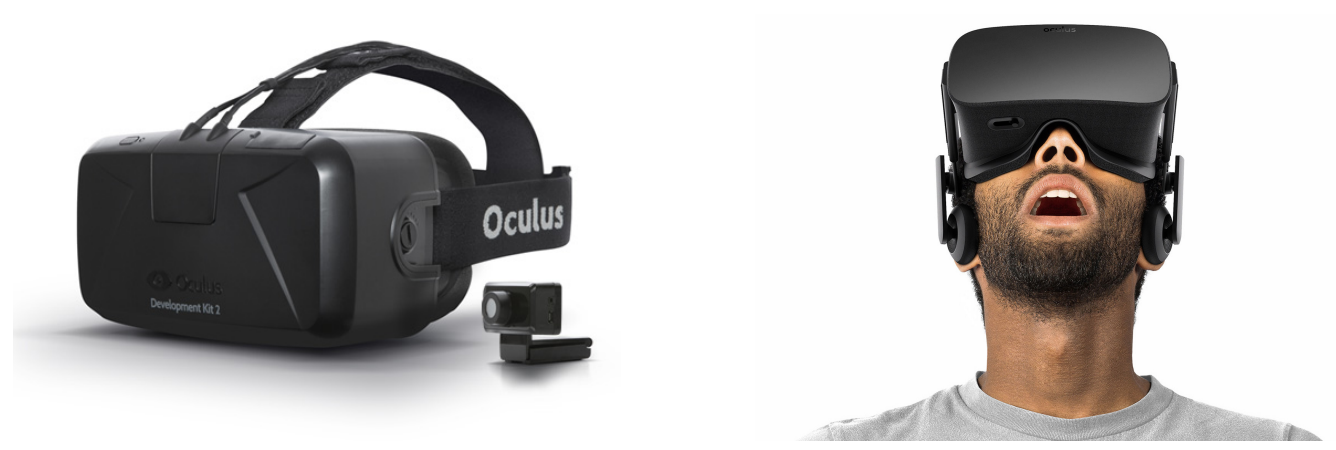

Figure 2.5: Oculus Rift DK2 head-mounted display [51], standalone and in-use. Images from http: //www . oculus. com.

field of view. These videos are easy to produce and consume, and have become widely available on sites such as YouTube [54], propelling them to become one of the most popular forms of content for consumer HMDs.

360 video has two major benefits. Firstly, when watched via HMD the viewer is able to look around at will, resulting in a strong sensation of immersion in the environment depicted in the 360 video. This is something which cannot be done immersively without both a fully enclosed display and head orientation tracking, both of which are typically provided by HMDs. Secondly 360 video is easy to capture, requiring only a modestly priced special-purpose camera (figure 2.6a) or combination camera mount (figure 2.6b) and a piece of post-processing software. Ease of capture and strong immersivity have especially lent themselves to videos of activities and places that would normally be difficult to experience or access, such as adventure sports and foreign travel.

The main drawback to 360 video is that capturing depth on a full panorama is difficult. If the viewer is expected not to move their head then a single stereo pair of cameras can be used and the images fed directly to each eye, but if the viewer is allowed to look around the scene at will then it is much more difficult to capture and display the depth of the entire scene wherever the viewer may be looking. 360 video is typically displayed as if it were either infinitely far away, or a fixed distance (typically around $15 \mathrm{~m}$ ) from the viewer, and thus one of the primary strong points of HMDs - the ability to display depth - is not utilized. 


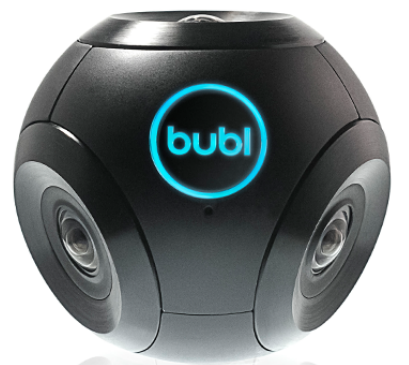

(a) Special-purpose 360 degree video camera. http://bublcam.com

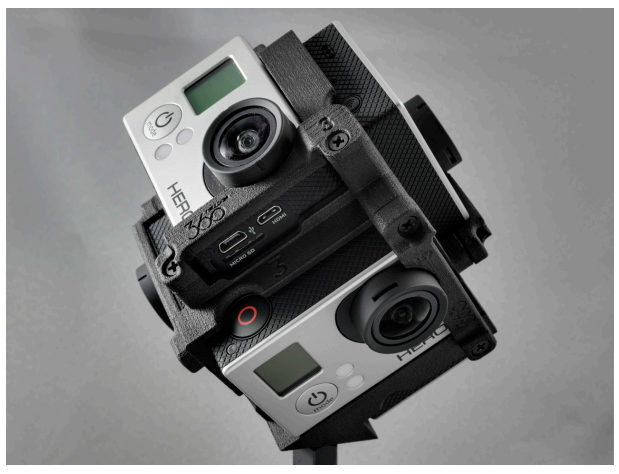

(b) Special-purpose mount combining standard cameras to create a 360 degree camera. http://freedom 360 .com

Figure 2.6: Examples of 360 degree panoramic video capturing hardware.

Capturing of 360 video is typically done in a manner similar to that described for IBL. Multiple standard or fisheye cameras are mounted together to jointly cover a full spherical field of view, and their individual video outputs are stitched together frame by frame to get a single 360 degree video as output. Because the camera positions are fixed relative to one another the stitching process is fairly straightforward, and once good positioning of the individual videos is achieved it can be maintained throughout. As for IBL the resulting video frames are usually stored in latlong format, but unlike IBL the frames are not stored in HDR format.

\section{Real-Time Image Based Lighting Techniques}

While performing traditional IBL in real-time is computationally prohibitively expensive, some of the computations can be done in advance resulting in real-time performance in certain scenarios. The most common method is to reduce the problem to two cases: pure specular reflection and pure diffuse reflection. Pure specular reflection simulates perfectly shiny materials such as metal (figure 2.7a) and glass. Pure diffuse reflection simulates perfectly matte materials such as plaster (figure 2.7b) and cardboard. By combining the two, other materials can be simulated such 


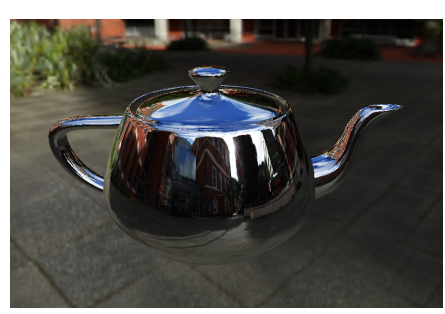

(a)

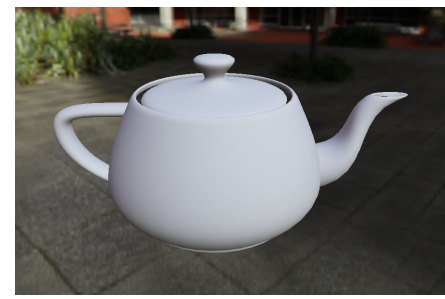

(b)

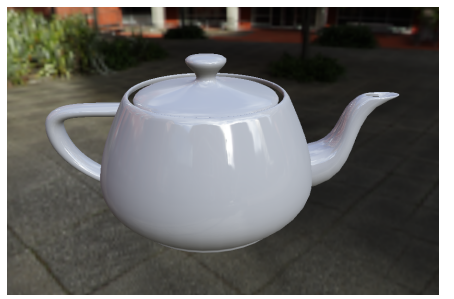

(c)

Figure 2.7: Pure specular reflection simulating metal (left), pure diffuse reflection simulating plaster (middle), specular and diffuse reflection combined to simulate porcelain (right).

as porcelain (figure 2.7c) and laminated wood.

For IBL in the case of pure specular reflection, the colour of the object at any point can be determined by reflecting a line from the virtual camera across its surface at that point (see figure 2.8a). The colour of the irradiance map in this direction is the colour the material appears to be at that point, so determining the material colour is a simple matter of a single lookup into the irradiance map. This can be done without any particular precomputation, and as such this technique is often used in $3 \mathrm{D}$ games to render pleasantly realistic shiny surfaces.

For diffuse reflection, the calculation is much more expensive. The colour at a given point on the surface is given by the formula (which can be derived directly from equation 2.1)

$$
\int_{\phi=0}^{\tau} \int_{\theta=0}^{\frac{\tau}{4}} I(n, \theta, \phi) \cos (\theta) \sin (\theta) d \theta d \phi
$$

where $n$ is the surface normal direction, $\phi$ is an azimuthal angle around the surface normal, $\theta$ is an elevation angle relative to the surface normal, $I$ is the intensity of incident light from the direction given by the angles $\theta$ and $\phi$ relative to $n$, and $\tau$ is $2 \pi$. The $\cos (\theta)$ term comes from the physical properties of diffuse reflection, and the $\sin (\theta)$ term accounts for the nonlinearity of the spherical integration.

The value of $I$ can be obtained directly from the irradiance map, but in a direct interpretation we still have to perform a two-dimensional integration over an entire 


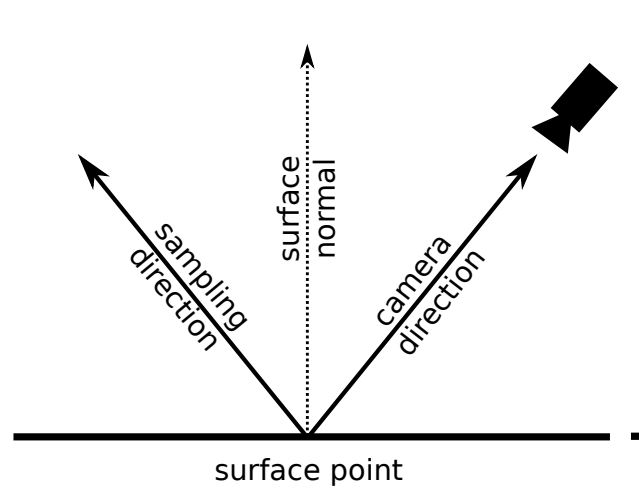

(a) Specular reflection.

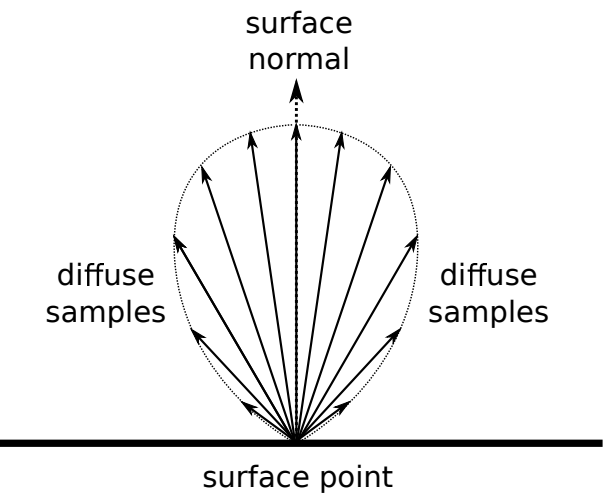

(b) Diffuse reflection.

Figure 2.8: Sample direction and weighting for specular and diffuse lighting calculations. Specular lighting only samples from one direction, whereas diffuse lighting samples from many but does not depend on camera position.

hemisphere to determine the final colour of the object at any specific point. However because the colour of diffuse reflection is constant with respect to viewer position and varies only with surface normal direction it is possible to compute it in advance for a specific set of surface normals. This is typically done at the same resolution as the irradiance map, resulting in a diffuse radiance map according to surface normal (such as figure 2.9b). The calculation of diffuse material colour (equation 2.2) then becomes a single lookup into this radiance map according to surface normal direction, allowing it to be done easily in real-time.

With this precomputation, we end up with two maps from direction to light intensity. The irradiance map stores the intensity of incident light according to direction of incidence. The diffuse radiance map stores the intensity of light radiated (in all directions) from a surface according to its surface normal orientation. Assuming these maps are of high enough resolution we can extrapolate the value at any direction to high precision with a small number of lookups, allowing us to render objects of the material types mentioned above in real-time using standard rasterization techniques.

While the result of this in the case of a single simple convex object such as a sphere can be considered accurate, it should be mentioned that the technique as for- 


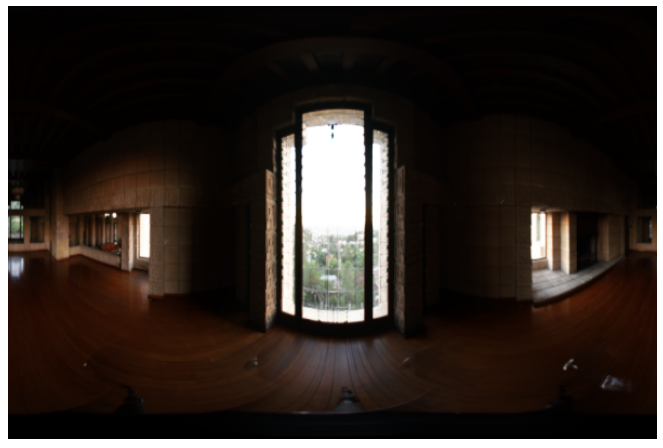

(a) Irradiance according to angle of incidence.

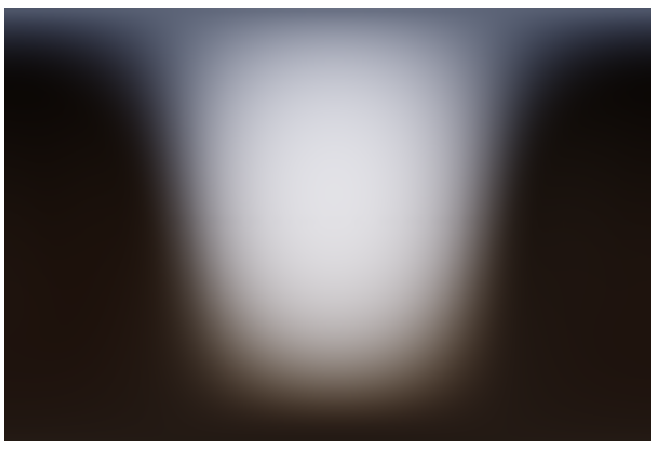

(b) Diffuse radiance according to surface normal direction.

Figure 2.9: An irradiance map of an indoor scene and the diffuse radiance map generated from it. Data is stored by azimuthal angle (horizontal position) and elevation angle (vertical position) according to some predetermined reference orientation. The irradiance map was obtained from http://gl.ict.usc.edu/Data/ HighResProbes/.

mulated here does not take into account occlusion of the environment. It is possible that in a scene with multiple simple objects or a single complex object, incident light at a certain point may be occluded by the geometry of the scene. In this case the assumptions used above will be incorrect, resulting in missing shadows, shading or reflections, and requiring other techniques to deal with this.

\section{Reduced Resolution Equivalence}

A result which will be used later on in this thesis stems from work by Chalmers et al. [9] on reducing the resolution of images used for IBL. With the intention of reducing the amount of storage space necessary to maintain a large database of images for use in IBL, it was found that the resolution of the images can often be greatly reduced without affecting the results after using them for IBL. For the lighting of highly specular (shiny, mirror-like) virtual objects it was shown that reducing the resolution of the irradiance map down to 300 pixels wide and 150 pixels tall gave 
a result that was perceptually indistinguishable from that using the full resolution map. For highly diffuse (dull, matte) objects, it was shown that the resolution can be reduced even further, down to around 80 pixels by 40 pixels without any noticable change in the final rendered output. These results were obtained using a physically based ray tracer, and the virtual objects were also shown to be indistinguishable from real objects in controlled conditions.

One of the novel contributions of this thesis is showing that this can be applied directly to real-time IBL.

\section{LDR-HDR Tonemapping}

One aspect that must be dealt with when displaying HDR images is that of tonemapping, determining what colour and intensity each part of the image should have when displayed on standard LDR media such as printed paper or a computer screen. Many methods for this have been proposed and studied, from simple static pixelwise transforms (such as the commonly-used Reinhard transform [42]) to complex transformations taking into account the entirety of the HDR image (such as gradient-domain dynamic range compression [12]), and no one method has been shown to be ideal. Often a simple exponential transform with maximum and minimum value cutoffs will be sufficient, but it requires the exponent, maximum and minimum values to be decided upon.

In our case we wish to consider the opposite transform, inverse tonemapping (also referred to simply as tonemapping), which converts an LDR image into an HDR image. Two papers give indications that we might be able to do this effectively. One is a paper by Akyüz et al. [3] in which the authors study the effect of displaying HDR images, LDR images, and converted LDR-HDR images on a special HDR display. They find that the simple pixelwise tonemapping transform

$$
L^{\prime}=k\left(\frac{L-L_{\min }}{L_{\max }-L_{\min }}\right)^{\gamma}
$$

is sufficient for effectively converting existing LDR images for HDR display. Here $L^{\prime}$ is output luminance, $k$ is the maximum desired output luminance, $L$ is the 
input luminance, $L_{\max }$ and $L_{\min }$ are the maximum and minimum possible input luminances, and $\gamma$ is a parameter.

The other is the previously-mentioned paper by Chalmers et al. [9] in which they determine that using the pixel-wise tonemapping equation of Landis [30] can be sufficient for converting LDR images for use in IBL. In many scenes, the result of IBL using a tonemapped LDR image was perceptually indistinguishable from the result using a pure HDR image.

These observations suggest that a simple tonemapping procedure could be sufficient for adapting LDR 360 degree video frames for use as HDR environment maps, and this will be explored further on in the thesis. 


\section{Chapter 3}

\section{Prior Work}

\section{Adding Virtual Objects to Real Scenes using IBL}

As mentioned in section 2.2, IBL has been used to convincingly render virtual objects into photographs of real scenes [11]. In the method of [11] a crude approximation of the scene is modeled via computer, and this approximate scene is then rendered twice using IBL, once with an additional virtual object inserted and once without it. The pixelwise difference between these two virtual scenes is then taken, and this difference is applied onto the original real-life photograph, transferring subtle effects such as soft shadows and minute reflections. The more geometric detail there is in the modeled scene the more accurate the end result will be, but because it is the difference between scenes with and without the object that is applied, exact details of the virtual modeled scene are not usually important. Inserting objects in this manner allows for effects to be captured that may normally be only barely perceptible, greatly increasing the perceived realism of the result.

To obtain this high level of realism there are three primary drawbacks. One is that the accurate rendering of the virtual scene takes a lot of time, so it is only suitable to offline or precomputed uses. Another is that the HDR environment image must be captured at the same time as the photograph, which can be unwieldy or impossible depending on the scene and scenario. The third is that the scene must be virtually modeled by hand. 
There has been some recent success in attempting to solve the problem of requiring an environment map by using machine learning along with a preexisting set of environment maps [22], and also in modelling the scene using roughly guided [22] or automatic means based on geometric assumptions [23]. These techniques work well when an appropriate environment map is in the database and geometry assumptions hold true, but can still be very inaccurate if either of these is not so.

\section{Real-Time IBL}

Real-time IBL techniques can be traced back to Blinn and Newell [6] who very early on described using images as textures to simulate reflection. Miller and Hoffmann [35] later pointed out that this can be considered a lighting technique and discussed how diffuse and glossy reflections could also be simulated by sampling from an environment image, as well as describing the method of precomputing a diffuse reflection map. Greene [14] continued on the topic of using projected environments for efficient lighting computation, and by 1999 Heidrich and Seidel [16] were able to demonstrate various real-time IBL techniques. Pre-filtering was used not only for diffuse reflections, but also to simulate low-gloss reflections such as can be seen on plastic and rough shiny surfaces.

Precomputed diffuse maps have already been explained in section 2.5, and precomputed glossy maps work in a similar manner, except instead of corresponding to surface normal direction the lookup is done according to the direction of specular reflection. Assuming a Phong [39] model for glossy reflection the input environment map is used to create a glossy reflection map. In fact many real-world materials have reflective properties depending primarily on surface normal direction and the direction of specular reflection, and Heidrich and Seidel [16] also discuss using this technique for other material types.

The primary drawback to the technique is that the material type must be known in advance, and a specialized lookup table of precomputed lighting integrals generated accordingly. When performing computations offline using a ray tracer reflective properties of any material type can be simulated, but using this real-time technique 
depends on only having a small variety of material types. To address this Kautz and McCool [24] emulate arbitrary reflective properties by formulating them as a combination of glossy reflections of varying levels of glossiness and with varying primary direction of reflection. This allows the application of IBL in real-time for arbitrary materials, so long as we have a full complement of precomputed maps for varying levels of glossiness.

Because of their simplicity and power, the techniques mentioned here form the core of many real-time IBL applications. Unger et al. [50] demonstrate real-time illumination using precomputed maps and HDR panoramas. Agusanto et al. [2] demonstrate its use for augmented reality. They suffer from the same drawback of requiring an environment image to be acquired and analyzed in advance, but show that IBL can be effectively performed in real-time using these techniques.

\section{Real-Time IBL using Spherical Harmonics}

One way to circumvent real-time IBL's need for prior analysis of the environment map was described and implemented by Ramamoorthi and Hanrahan [41]. They discovered that accurate diffuse lighting can be achieved by representing the diffuse radiance map as a linear combination of spherical harmonic ${ }^{1}$ functions, and that restricting coefficients to as few as the 9 simplest basis functions was sufficient. They determined that these 9 diffuse radiance coefficients are equivalent to the first 9 coefficients of the spherical harmonic representation of the irradiance map, and thus calculate each coefficient directly by multiplying the irradiance map by the appropriate spherical harmonic basis function and integrating over the sphere:

$$
C_{Y}=\int_{\phi=0}^{\tau} \int_{\theta=-\frac{\tau}{4}}^{\frac{\tau}{4}} I(\theta, \phi) Y(\theta, \phi) \cos (\theta) d \theta d \phi
$$

where $C_{Y}$ is the coefficient corresponding to spherical harmonic basis function $Y$ and the rest of the terms are as in equation 2.2. The $\cos (\theta)$ term adjusts for the varying pixel density of an irradiance map in latlong format. This calculation is

\footnotetext{
${ }^{1}$ an orthonormal set of functions that form a basis for the set of all functions on a spherical domain
} 
similar to that calculating the diffuse radiance map directly, except instead of being applied $W \cdot H$ times for a $W$ by $H$ irradiance map it is only applied 9 times, and thus the complexity of the operation can be considered $\mathrm{O}\left(9 N^{2}\right)$ instead of $\mathrm{O}\left(N^{4}\right)$ in irradiance map size.

This technique was shown by King [25] to be able to be effectively applied in real-time using commonly available dedicated graphics hardware, and has become one of the standard techniques for real-time IBL used in 3D games. By combining this technique with simple specular reflection mapping, many real-world materials can be simulated efficiently and effectively. Typically modern games will capture the environment at a small number of positions and use these techniques to calculate diffuse and specular reflection of dynamic virtual scenes in real-time. This is especially effective when capturing near a single object such as a player avatar or vehicle under the player's control which will attract more focus than other objects (such as a car in a racing game), and can thus have a great impact on perceived rendering quality.

It has also been explored as an augmented reality technique [38] but this still suffers from the drawback of needing the entire environment image to be provided, which is not usually practical for AR.

\section{Real-Time IBL using HDR video}

Looking into the problem of irradiance map acquisition Unger et al. use an HDR video camera with attached light probe (see figure 3.1) to efficiently capture a great number of environment maps. They use this for enhanced capture of lighting information in the entire volume of a large static scene [48] allowing virtual objects to be believably placed at any position in any photograph of the scene, and later they attach a standard camera to the HDR camera and use the environment map captured by the HDR camera to believably insert a virtual object directly into the video captured by the standard camera [49]. This can be considered an extension of Debevec's work $[10,11]$ from photographs to video, and from single point environment captures to multiple spatially distributed environment captures. 


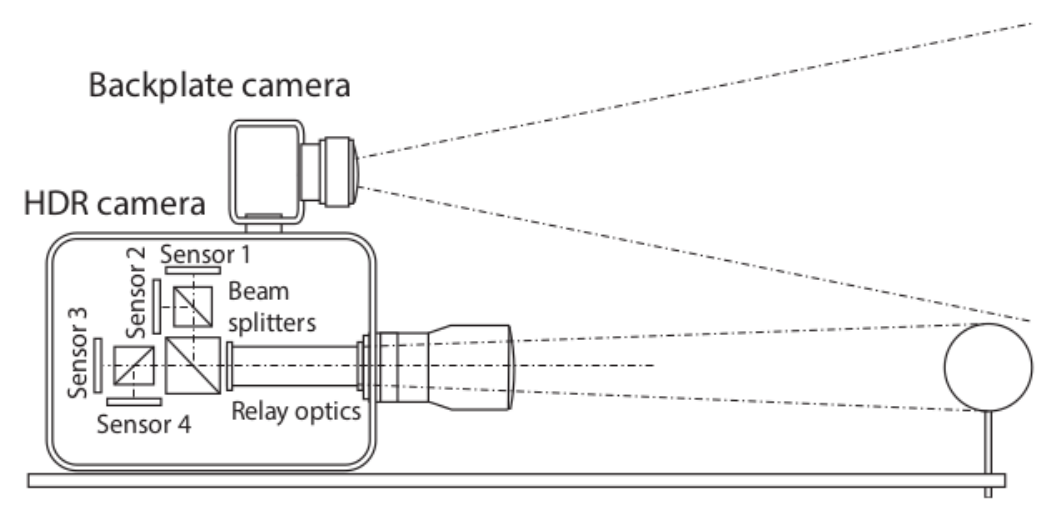

Figure 3.1: Diagram of the setup for HDR environment capture used in [48], [49] and [29]. An HDR video camera captures the environment via an affixed light probe. A standard LDR camera can be mounted above to capture plain video for later augmentation.

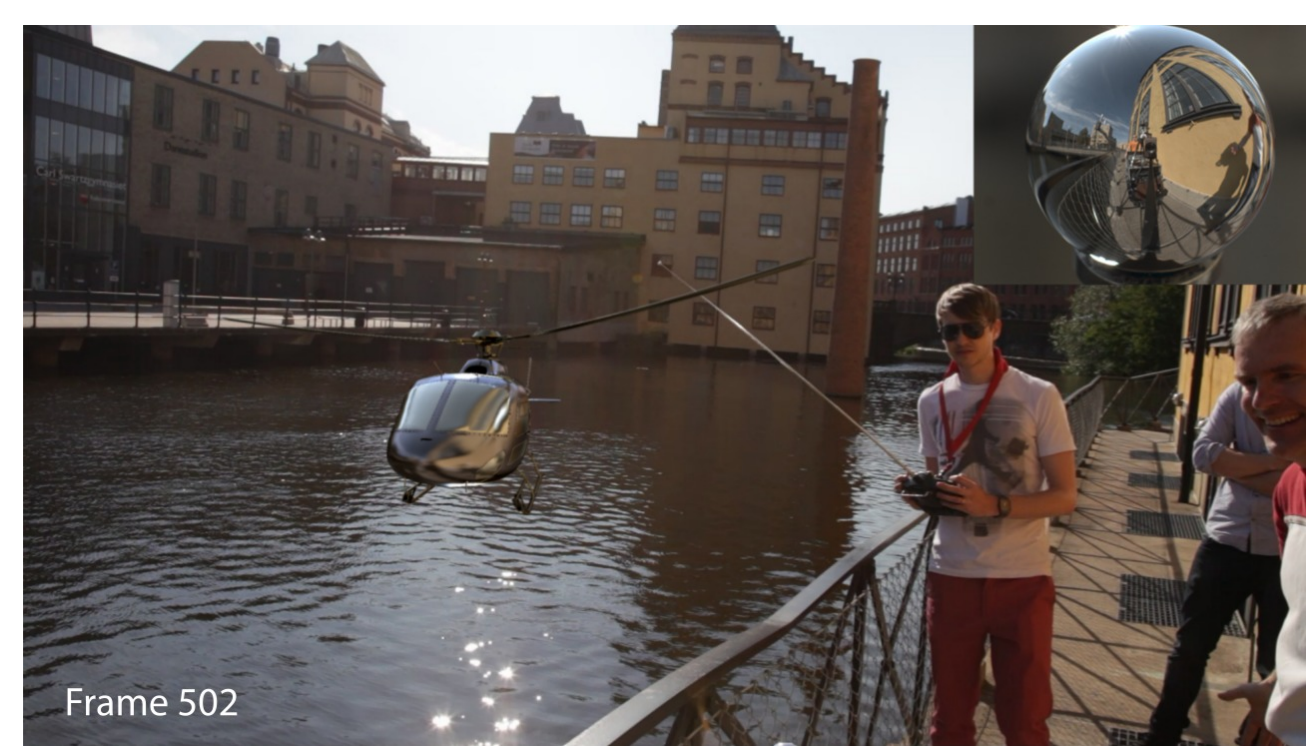

Figure 3.2: Results from [29], augmenting captured real-life video with a virtual helicopter. Because HDR environment maps are captured simultaneously with the standard video, very high quality results can be obtained. Here the light probe image for this frame captured by the HDR video camera is displayed in the top right. 
Using the same capturing device as [49], Kronander et al. [29] show that a virtual object can be rendered into the LDR scene in real-time using bidirectional importance sampling [8]. This calculates IBL directly, but instead of sampling over an entire hemisphere, samples are concentrated on the areas likely to have greatest effect on the colour of the object as viewed from the camera position. Effectively the reflectance function of the object is multiplied by the distribution of incident light, and the lighting integral is estimated from a small number of samples according to the weighting of this combined distribution. Assuming the lighting does not change significantly between frames, this distribution will vary slowly and can thus be updated fairly efficiently each frame.

This method provides very good results (see figure 3.2), and can handle materials with complex reflection properties. Its primary drawbacks are the need for uncommon special-purpose hardware for environment capture, and a complex rendering procedure which makes implementation difficult.

\section{Real-Time IBL using Omnidirectional Video}

Recently Michiels et al. [34] demonstrate the usage of captured $360^{\circ}$ video to render virtual objects into a reconstructed scene. Their panoramic video capturing hardware is fixed to a vehicle which is driven through a real life environment. They reconstruct the environment from the resulting video, also determining the camera position in this environment at each frame. Using this information they are able to render virtual objects into the scene according to the position of the object in the reconstructed 3D space. The most appropriate omnidirectional camera image is used as the environment map for IBL.

Their IBL technique is similar to that using spherical harmonics, except it uses spherical radial basis functions [52] instead of a spherical harmonic basis. This allows glossy reflection to be approximated in a similar manner to diffuse reflection approximation using spherical harmonics. Rendering of virtual objects can then be done in real-time for a wide variety of material types.

There are however still several major drawbacks to this technique. The SRBF 

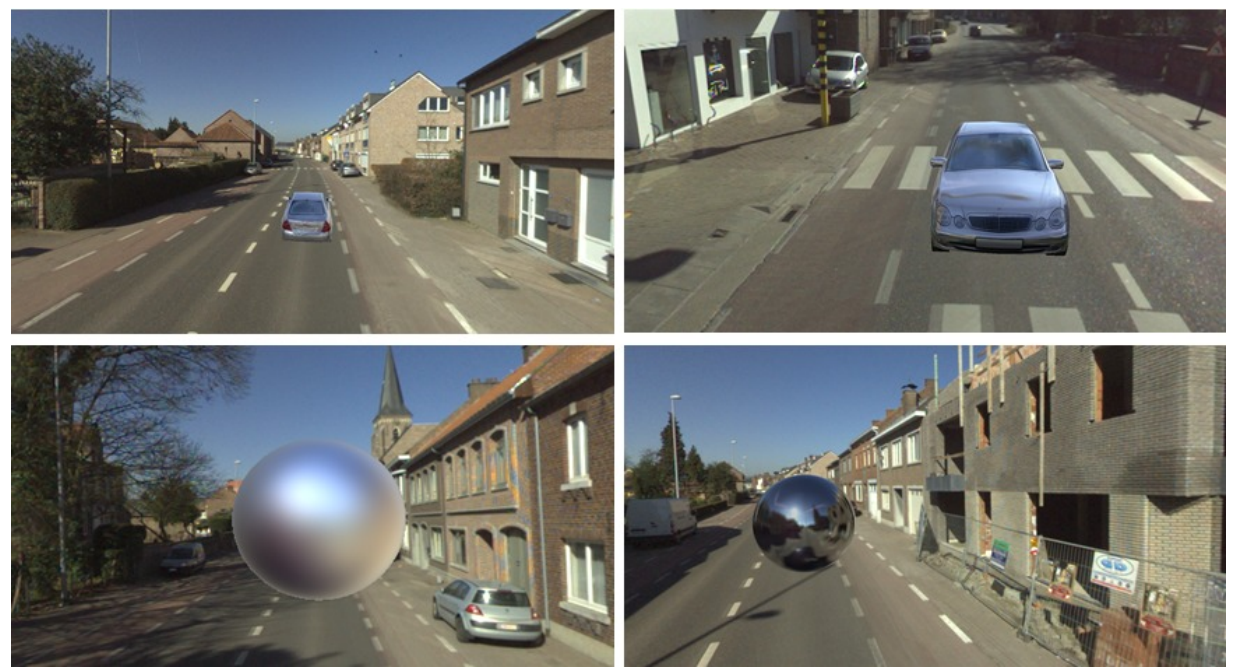

Figure 3.3: Results from [34]. Lack of HDR environment maps leads to inaccurate and thus somewhat unconvincing lighting, as can be seen with the lower right sphere appearing darker than the surroundings, and the upper right car appearing more blue than would be expected from looking at the environment.

conversion is not done in real-time and thus requires a preprocessing step in addition to the preprocessing necessary to determine the $3 \mathrm{D}$ position of the environment captures, meaning the technique is suitable for MR or VR but not real-time AR. The implementation is complex (and in the cited paper incomplete) and it is not clear how necessary the scene reconstruction step is. Primarily however the lack of HDR input means that their rendered result is not entirely convincing (see figure 3.3).

The following thesis can be considered as attempting to alleviate or ameliorate all of these drawbacks, allowing for accurate real-time IBL using live LDR $360^{\circ}$ video.

\section{Filtered Importance Sampling}

Filtered Importance Sampling is a technique for reducing sampling burden by prefiltering the environment map. The difference between this technique and the pre- 

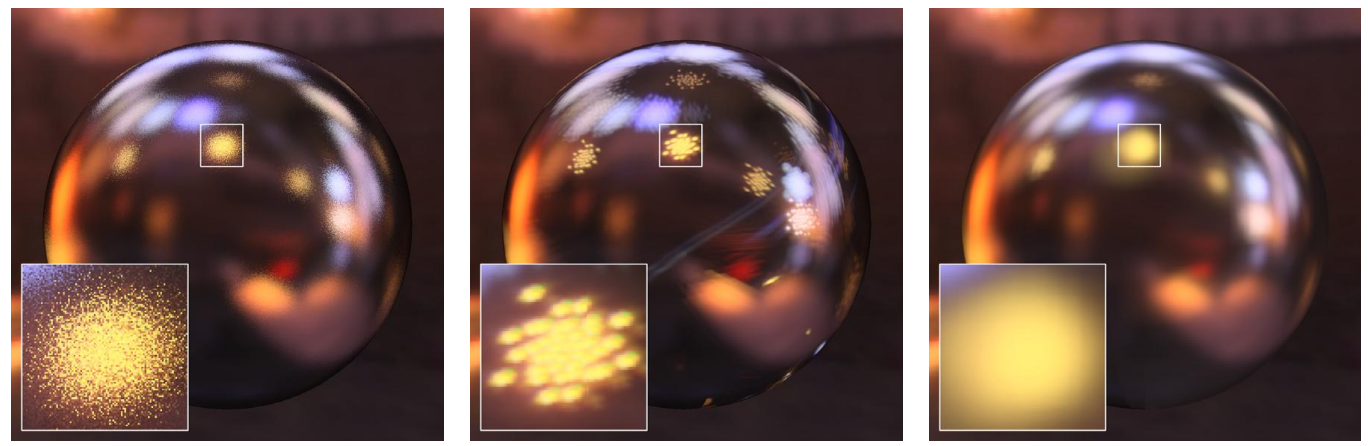

Figure 3.4: Results of filtered importance sampling (right) compared to random importance sampling (left) and static importance sampling (middle). Static sampling has obvious visual artifacts according to the sampling pattern, while random sampling produces visual noise even with a very high number of samples. In comparison results using filtered importance sampling are smooth even with a small number of static samples. Images from [28].

viously mentioned techniques involving precomputation of integrals is that instead of relying on computations according to reflectance function the filtering is based on standard mipmapping procedure. Mipmap filtering is implemented and commonly used in all modern graphics hardware, and can thus be performed quickly and efficiently.

Filtered importance sampling was briefly described by King [25], and discussed in a general case very early on by Miller and Hoffman [35]. Instead of sampling from the environment map directly, an appropriate resolution mipmap is sampled from according to the distance between samples. If the sampling pattern and mipmap level are chosen correctly this should give a result similar to direct sampling with a much higher number of samples (see figure 3.4).

Křivánek and Colbert show that filtered importance sampling can be used for real-time IBL [28], including for complex reflectance functions. Their displayed results are not entirely convincing (see figure 3.5), but performance is good, rendering around $75 \mathrm{~Hz}$ on an Nvidia 8800 GTX graphics card equivalent to the low end GeForce 730 used later on in this thesis. 

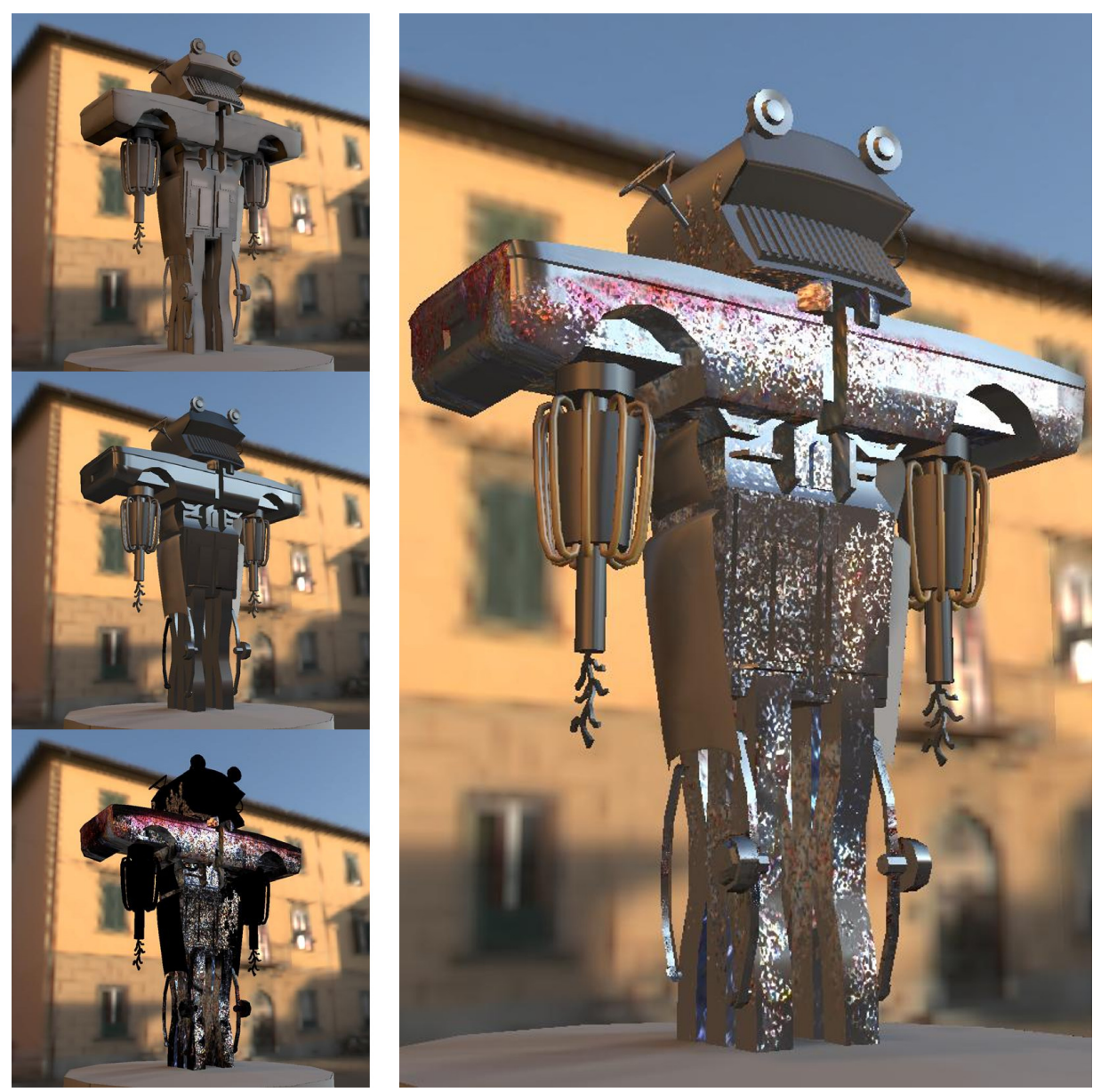

Figure 3.5: Results from [28]. On the left are (from top to bottom) diffuse lighting, glossy lighting and lighting using a complex reflectance function, all calculated using filtered importance sampling. They are combined in the result on the right. 


\section{Chapter 4}

\section{System Solution}

The primary goal of this thesis is to develop a novel pipeline for real-time image based lighting using 360 degree panoramic video as the environment image. The system should be capable of using live LDR 360 degree video, and rendering to head mounted display at comfortable framerates. On the Oculus Rift DK2 [51] headset used for testing, comfortable viewing requires that stereographic output be rendered at a stable framerate of at least $60 \mathrm{~Hz}$ [53] at its recommended output resolution of $1182 \times 1464$ pixels per eye.

Development of the system solution is separated into two sections. In the first section a basic real-time IBL system is developed, operating using standard HDR envrionment maps. In the second section this system is adapted to operate using LDR 360 degree video frames as environment maps instead.

\section{Basic Real-Time Image Based Lighting}

In this section a system is created for the application of image based lighting in realtime using consumer-grade graphics hardware and with no precomputation required. This system uses mipmap-based filtering to enable the simulation of arbitrary classes of reflection effects by using an appropriate sampling pattern. For this implementation only pure specular reflection, glossy specular reflection, and diffuse reflection are considered, but the described system can be easily extended to apply to other 
reflectance functions as necessary.

The method of mipmap-filtered sampling is shown to be capable of creating accurate diffuse radiance maps in real-time without precomputation, which can further speed up rendering, and this is used in the final system for this purpose. The constructed system is designed to be adaptable between frameworks, and able to be optimized for either speed or quality if used on low-end hardware. On consumergrade desktop hardware we find that a prototype implementation is able to run with high visual quality at very high resolutions and framerates, enabling advanced applications such as augmented reality via head-mounted display.

\section{Problem Description}

For the initial implementation we constrain the problem to that of real-time image based lighting of a single virtual object using a previously-captured HDR environment map. The resulting program must run on a consumer-grade machine, and be capable of rendering to an Oculus Rift DK2 [51] HMD at over 60Hz. Visual quality should be at least believably realistic, and ideally perceptually indistinguishable from reality. It should be able to run immediately on real-time input, and as such no analysis of the environment map is allowed prior to rendering.

\section{Basic System Solution}

The preliminary real-time IBL solution can be described in three stages.

\section{Mipmap Filtering}

Stage one simply involves calculating mipmaps for the given HDR environment map. Input is the original HDR environment map, and output is a mipmapped HDR environment map which can be considered to be a set of HDR environment images representing the same environment map at halving horizontal and vertical resolution all the way down to 2 pixels by 1 pixel. So for example if the input is a single 2048x1024 image, output will be eleven images representing the input environment 
map scaled to the sizes $2048 \times 1024$ (unchanged), 1024x512, 512x256, 256x128, $128 \times 64,64 \times 32,32 \times 16,16 \times 8,8 \times 4,4 \times 2$ and $2 \times 1$.

\section{Diffuse Radiance Map}

Stage two involves calculating a diffuse radiance map from the previous set of environment images. Previously Chalmers [9] showed that using images down to 80x40 for diffuse radiance maps can be perceptually indistinguishable from reality, and from this result we hypothesize that the diffuse radiance map can be computed with no loss in quality using at most the $128 \times 64$ image from the previous stage. As the numerical computation of diffuse radiance (equation 2.2 ) is highly parallelizable, there should be no problem running it at low resolution in real-time on a consumer-grade GPU.

The output of stage two is thus a low resolution diffuse radiance map. The resolution of this map can be lowered to decrease computation time (and thus increase framerate), and can be raised to increase fidelity depending on hardware capability. As resolution increases the result will approach that of offline ray-traced IBL, which has been shown $[11,9]$ to be capable of producing results indistinguishable from reality. Furthermore there should be some limit [9] beyond which further improvement in output fidelity is imperceptible to humans, and so real-time IBL rendering results indistinguishable from reality may be achievable, at least for materials which can be modeled by a combination of diffuse and pure specular reflection only.

\section{Rasterization}

Stage three involves rendering a virtual object using the scaled environment maps from stage one as prefiltered irradiance maps, and the precomputed diffuse radiance map from stage two for convenience. As a diffuse lighting component is present in almost any solid real-world material, stage two can be assumed to be worthwhile in the vast majority of cases. In addition to the outputs of stages one and two, input to stage three includes details about the geometry and material of the virtual object to be rendered, the orientation of the object relative to the environment depicted in 
the input environment map, and the position and orientation of the viewer relative to the virtual object.

Using the position and orientation of viewer and object as well as details of the geometry of the object, the object can be rendered using standard rasterization techniques. Given the reflectance properties of the material to be rendered, the fragment shader step of GPU rasterization (determining the colour of the object at each individual pixel) can perform filtered importance sampling to apply IBL according to the material. Combining with diffuse reflection using the diffuse radiance map and specular reflection using direct specular lookup allows us to accurately and efficiently simulate a wide variety of real-life materials.

As a benefit to this technique, supporting a large number of material types should not increase computational complexity. Only the sampling pattern would need to be modified, and this can be supported as a choice inside the fragment shader. Computation time will only increase if more than one reflection effect is to be combined.

The output of this third stage is an HDR image of a virtual object rendered using IBL according to the input HDR environment map. This image can be combined with a perspective view of the original environment map to add the virtual object to the scene, or used for some other purpose.

\section{Prototype Implementation}

In order to test this system, a software prototype was developed that can take an input HDR environment map and a 3D model, and display the 3D model in front of the background environment with accurate lighting as if viewed from nearby.

The prototype was implemented in C++ using OpenGL 3.3 for rendering. Images were loaded using the FreeImagePlus library [13], and 3D models were imported using the Open Asset Import Library [46].

\section{Program Structure}

For stage one of the implementation, the HDR environment image is loaded into a floating point OpenGL texture using FreeImagePlus. Mipmaps are then generated 
automatically with the appropriate OpenGL call.

For stage two a separate floating point texture is created, and this is rendered onto with a custom fragment shader which takes the mipmapped HDR envionment image as input. In the case of this prototype the diffuse radiance map size is hardcoded, but this could easily be variable. The shader code first determines which pixel of the output texture it is rendering to, then performs the integration of equation 2.2 numerically using an appropriate resolution mipmap level of the input irradiance map. The output texture can then be used as the diffuse radiance map for the next stage of the pipeline.

For the final output another floating point texture is created, allowing the result to be rendered in HDR. This step is not strictly necessary and output could directly be tonemapped to LDR, but is useful for testing.

Before stage three, a perspective view of the input environment map is rendered onto the output texture. This is done by taking a simple planar projection using the current camera position and sampling from the appropriate direction according to the input environment map. Camera position is partially hardcoded and partially determined by user input.

For stage three the virtual model is loaded into OpenGL memory as a set of triangles using the Open Asset Import Library, and these triangles are rasterized on top of the previously rendered perspective view of the environment. The fragment shader is set up to perform IBL using provided inputs of mipmapped HDR irradiance map, HDR diffuse radiance map, material diffuse reflection proportion, material specular reflection proportion, and material roughness, as well as surface normal and camera directions given by the vertex shader.

Diffuse and pure specular reflection (in the case when roughness is zero) calculations are performed as lookups into the diffuse radiance map according to surface normal, and irradiance map according to the direction of specular reflection. It would also be possible to add other effects such as emissivity or translucency here, but these effects are fairly well understood and would not change any of the methods used, so will be considered out of scope for this and later implementations.

If roughness is not zero, glossy reflection is calculated using mipmap-filtered 
sampling with a specific sampling distribution chosen both for applicability to glossy reflection and for ease of filtering via mipmaps. The first step of this calculation determines the size of a cone in which the main proportion of glossy lighting contribution resides, by assuming that the most important region is between the primary direction of reflection and the angle at which the importance of incoming light falls to exactly half its maximum. For this implementation the Phong model [39] is used, for which this calculation is not difficult.

Having determined the approximate size of the important area contributing to glossy reflection, we then form six concentric rings of six sampling points per ring. Three rings are placed evenly inside the cone of importance, and three rings are placed evenly outside it. This arrangement is somewhat arbitrary, but was chosen both to make it easy to determine filtering size and because it was found experimentally to give good results for this particular reflectance function. Sampling is done per ring, with filtering size fixed according to the distance between sample points. This filtering size parameter is used to make texture lookups using standard harwaresupported trilinear filtering, which automatically linearly extrapolates between the closest four pixels of the irradiance map to the sampling point, as well as the two nearest mipmap levels according to desired filter size.

In this way samples are integrated according to the weighting determined by the reflectance model with fairly accurate results (see figure 4.2). Glossy (or pure if roughness is zero) specular reflection and diffuse radiance are then combined in proportion according to the given input material parameters, resulting in the final output colour for this fragment. The use of 36 samples per fragment was found to be acceptable for real-time use (see figure 4.1), and it is possible that fewer could be used while maintaining visual accuracy.

As a final stage the output is rendered onto the final display via a simple tonemapping shader, converting from HDR to LDR according to a user-variable exposure parameter.

The end result of this prototype implementation is a pipeline taking as input an HDR environment map and a 3D model with specified material parameters, and outputting accurate real-time perspective renderings of the model placed inside and 


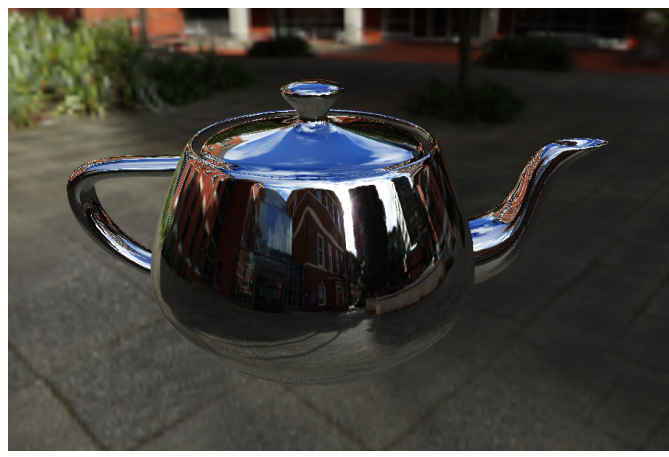

(a) Pure specular reflection. $7 \mathrm{~ms} / 1.1 \mathrm{~ms}$.

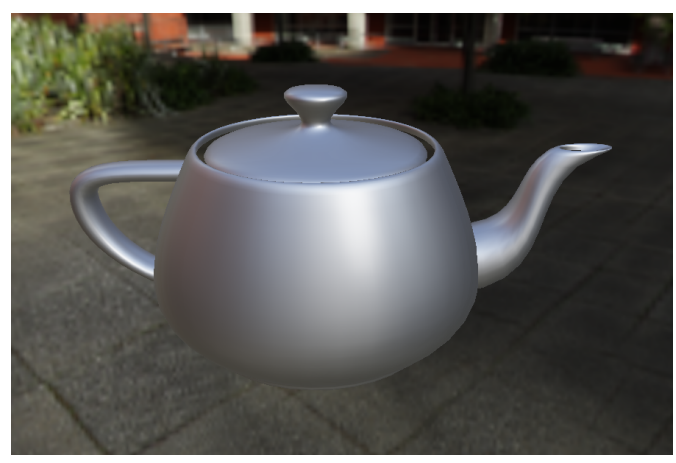

(c) Glossy specular reflection. $15 \mathrm{~ms} / 2.0 \mathrm{~ms}$.

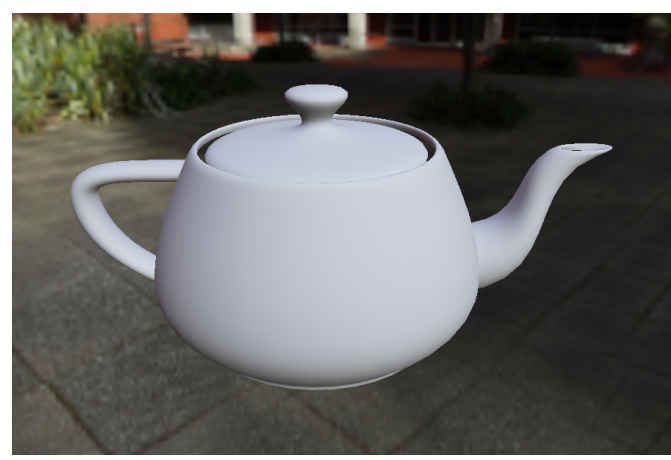

(b) Pure diffuse reflection. $7 \mathrm{~ms} / 1.1 \mathrm{~ms}$.

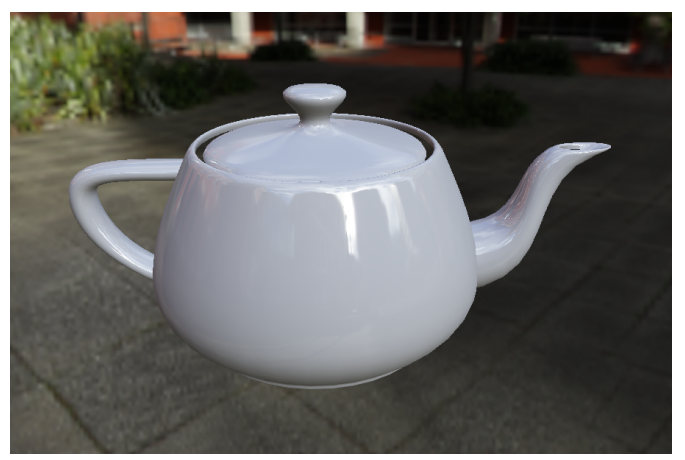

(d) All reflection types. 20ms / 3.0ms.

Figure 4.1: A virtual teapot rendered in real-time at 1280x720 resolution. Frame rendering time for a low end Nvidia GeForce 730 and high end Nvidia GeForce 980 graphics card is as indicated.

realistically lit by the environment depicted in the input environment map. Model and image loading and memory allocation is done as an initial step, but all rendering tasks including diffuse radiance map generation are done every frame, discarding any results from the previous frame. As such the system is theoretically capable of working with real-time input, so long as that input can be converted and sent to graphics memory quickly enough. 

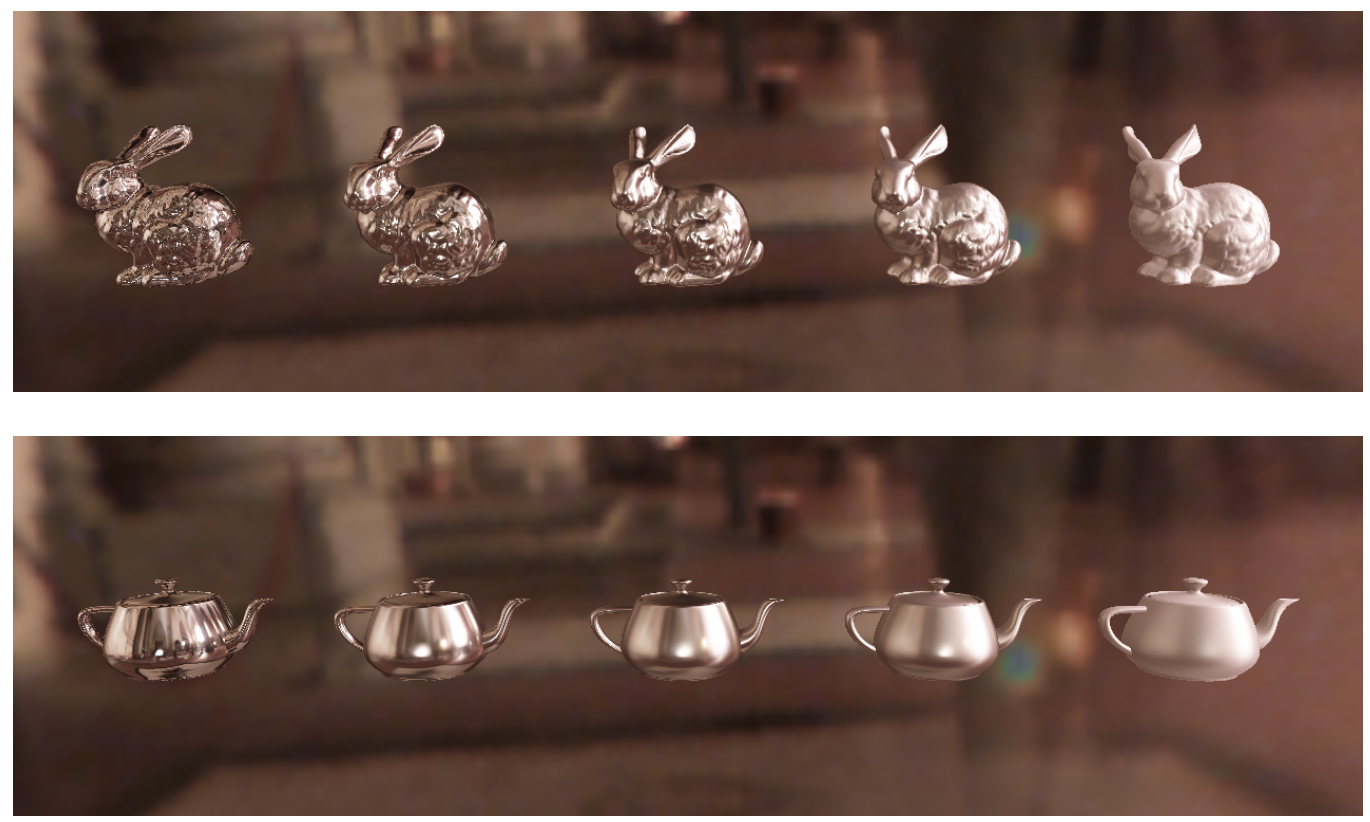

Figure 4.2: Glossy specular reflection for various lobe sizes determined by a roughness parameter of, from left to right: $0.01,0.05,0.1,0.2,0.5$.

\section{Results}

The real-time IBL prototype was tested on several consumer level graphics cards, with results recorded for the low end Nvidia GeForce 730 and high end Nvidia Gefore 980 cards. The prototype was found to run at high real-time framerates (see figure 4.1) exceeding $50 \mathrm{~Hz}$ at standard $1280 \times 720$ resolution even on the low end graphics card. The high end graphics card was exceedingly fast, rendering at up to $500 \mathrm{~Hz}$, and able to easily display to the Oculus Rift DK2 at the required $60 \mathrm{~Hz}$.

As computation is done in the fragment shader, computation time depends not on the number of virtual objects present in the scene, but the proportion of the screen taken up by virtual objects. In the tests done in figure 4.1, the majority of the view was taken up, so scenes with more distant objects will be rendered even more quickly.

Glossy lighting (figure 4.2) appeared realistic at various levels of roughness, and mipmap-filtered sampling performed as expected in this case. Although other com- 
plex reflectance functions were not tested, they should be possible to simulate using the same principle. Even with only glossy reflection, it has been shown that combining glossy reflections of varying roughness and primary reflection direction can simulate many other reflectance functions [24].

Diffuse radiance map generation and usage was tested at various lowered resolutions (see figures 4.3 and 4.4), and the lowered resolution was found to only have a significant effect at $32 \times 16$ resolution and below. Diffuse radiance maps calculated at $64 \times 32$ resolution were not found to provide significantly different results from maps calculated at full $1024 \times 512$ resolution, but whereas the full resolution diffuse map took between 3 and 60 seconds to calculate depending on graphics processor, the $64 \times 32$ resolution version took just 1.5 milliseconds on the GeForce 730 and 1 millisecond on the GeForce 980. In practice when objects were viewed via HMD, even lowering the diffuse map resolution to $32 \times 16$ did not seem to have a significant effect on visual quality.

As the equations used are the same as those used when performing offline calculations, the results obtained here indicate that photorealistic results can be achieved in real-time using even low end modern graphics hardware.

While the individual parts of this pipeline are not novel, their possible application in this way does not appear to be widely known. It is even commonly stated [50][34] that computing diffuse IBL lighting in real-time is not possible due to the complexity of the operation. This is shown here to be untrue. It has been an assumption that the only way to perform IBL in real-time is to rely on converting the irradiance map into other formats such as spherical harmonics [41][38] or spherical radial basis functions [34], or to precompute reflectance or radiance maps according to the properties of the material [24][2]. Previous real-time systems using importance sampling have made use of complex methods such as bidirectional importance sampling requiring temporal coherence of the input environment [29]. This is shown here to be unnecessary. Standard IBL techniques can be applied in real-time using consumer-grade graphics hardware and standard GPU programming techniques. 


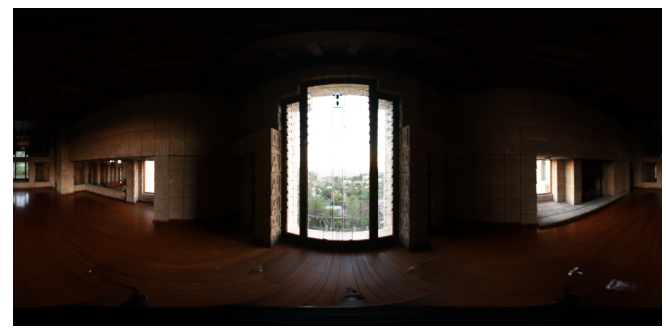

(a) HDR input image

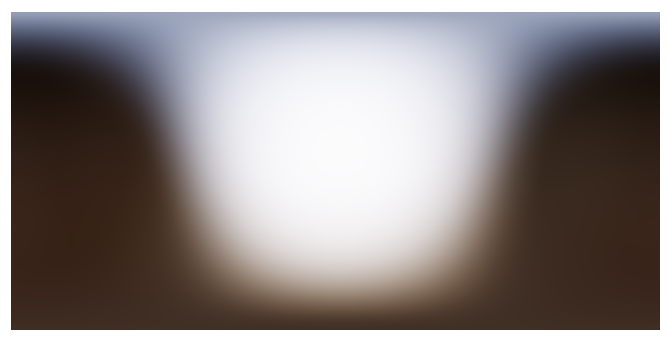

(c) $128 \times 64(3 \mathrm{~ms}, 15 \mathrm{~ms})$

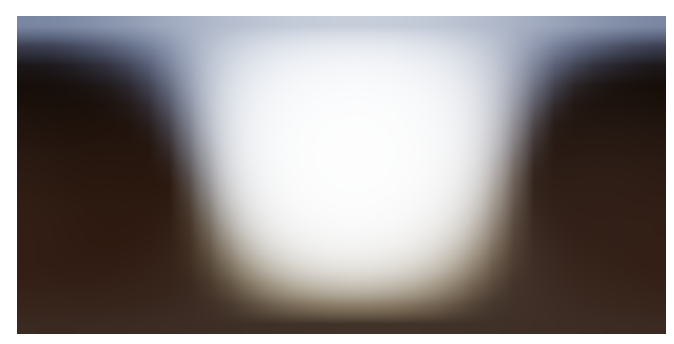

(e) $32 \times 16(0.5 \mathrm{~ms}, 0.5 \mathrm{~ms})$

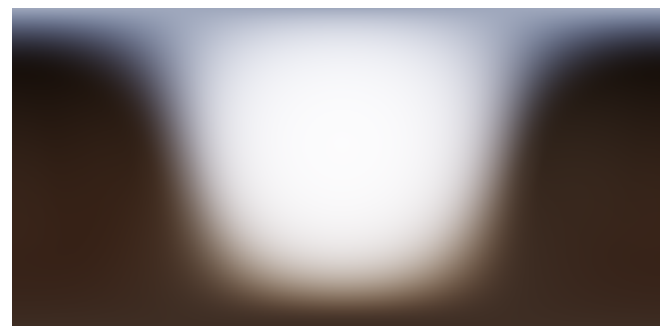

(b) $1024 \times 512(3 \mathrm{~s}, 60 \mathrm{~s})$

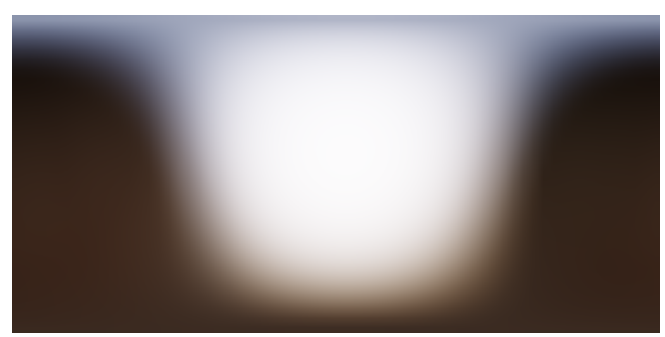

(d) $64 \times 32(1 \mathrm{~ms}, 1.5 \mathrm{~ms})$

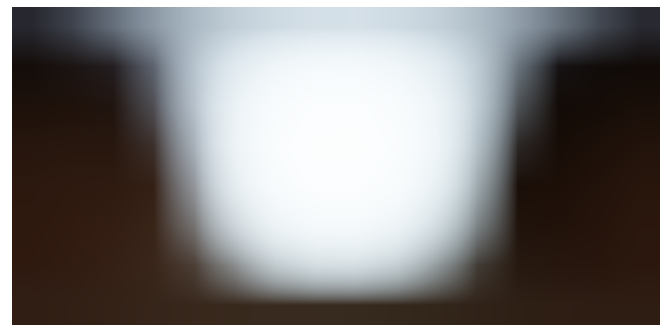

(f) $16 \times 8(0.3 \mathrm{~ms}, 0.3 \mathrm{~ms})$

Figure 4.3: Diffuse radiance maps computed at various resolutions. Generation times on a high end Nvidia GeForce 980 and low end Nvidia GeForce 730 are given in parentheses. Maps are displayed and sampled using hardware bilinear filtering. Colour inaccuracy and pixelization only become apparent at 32x16 resolution and below. 


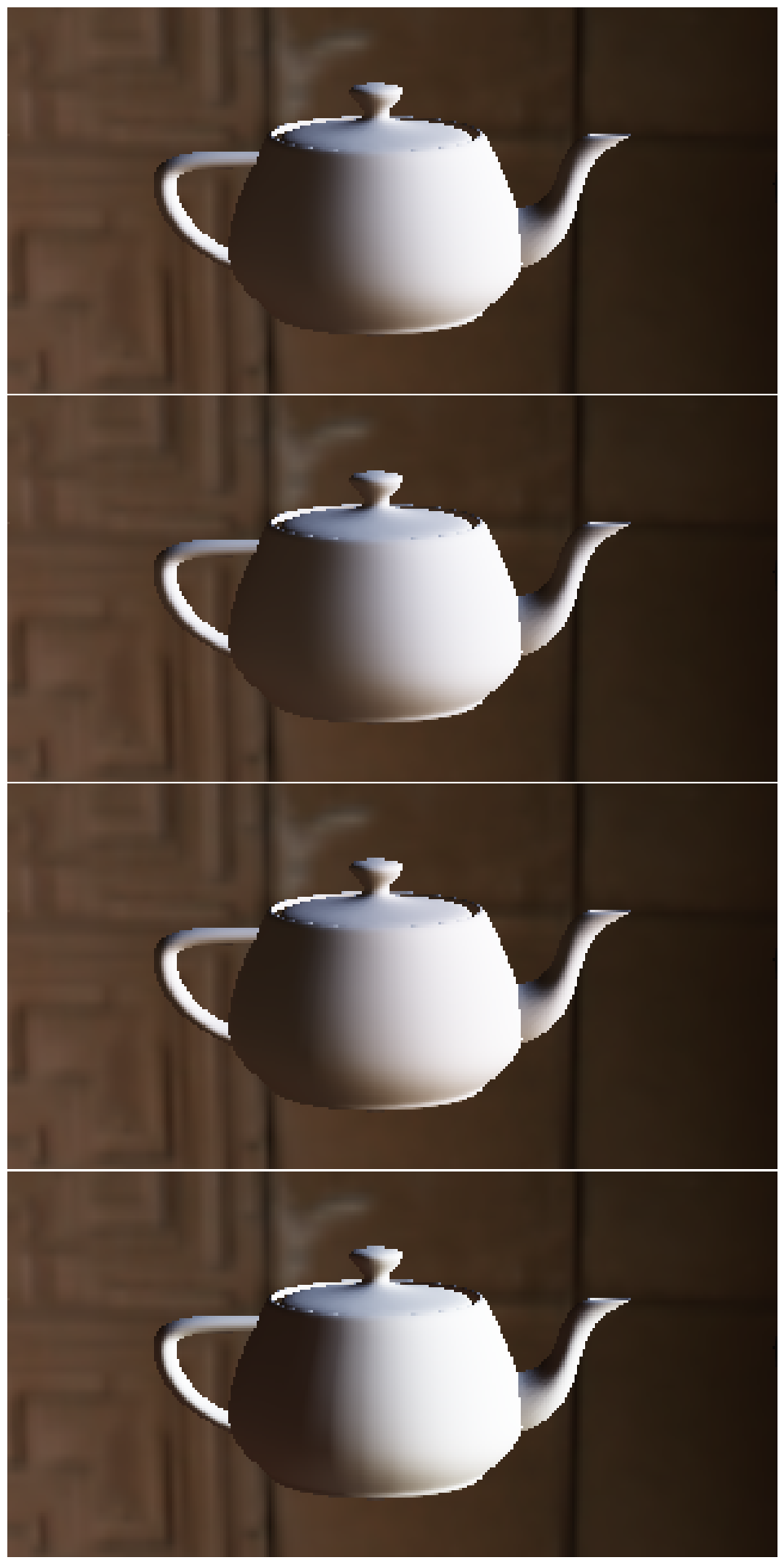

Figure 4.4: Diffuse lighting using generated diffuse maps of size (from top to bot-

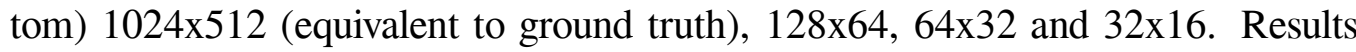
only begin to differ from ground truth around $64 \times 32$ resolution, with the difference only becoming pronounced around 32x16. 


\section{Real-Time IBL for 360 Degree Panoramic Video}

The result of the development in section 4.1 is a system for performing real-time image based lighting of virtual objects according to a given HDR environment map and without any precomputation. Performance and results of the prototype implementation were good, but the system still suffers from the problem of requiring a suitable HDR environment map to be provided. As these are commonly difficult and time consuming to capture and refine, this leaves the system without suitable real-world input to demonstrate its strengths. If an HDR environment map must be captured and processed beforehand, there is little practical benefit in avoiding precomputation of radiance and reflectance maps.

As it happens however the rise in popularity of 360 degree panoramic video has provided us with nearly the ideal input for this type of system. If the problems associated with using an LDR environment map are overcome, as the results of [9] and [3] mentioned in section 2.7 suggest is possible, the benefits of using the previously developed solution should become clear.

\section{Problem Description}

Given live LDR 360 degree video input of at least 1280x720 pixel resolution and $24 \mathrm{~Hz}$ framerate, believably render virtual objects into a perspective view of the depicted scene in real-time and at an output resolution of at least 1280x720 pixels and framerate of at least $50 \mathrm{~Hz}$. Targets should be achieved using consumer-grade dedicated graphics hardware. Ideally the result should be able to be displayed to an Oculus Rift DK2 HMD at the $60 \mathrm{~Hz}$ and $1182 \times 1464$ pixels per eye recommended for this device.

\section{System Solution}

For the most part, the system solution is similar to that proposed in the previous section. The only significant change is the addition of an LDR-HDR tonemapping stage. The final system is as shown in figure 4.5. 


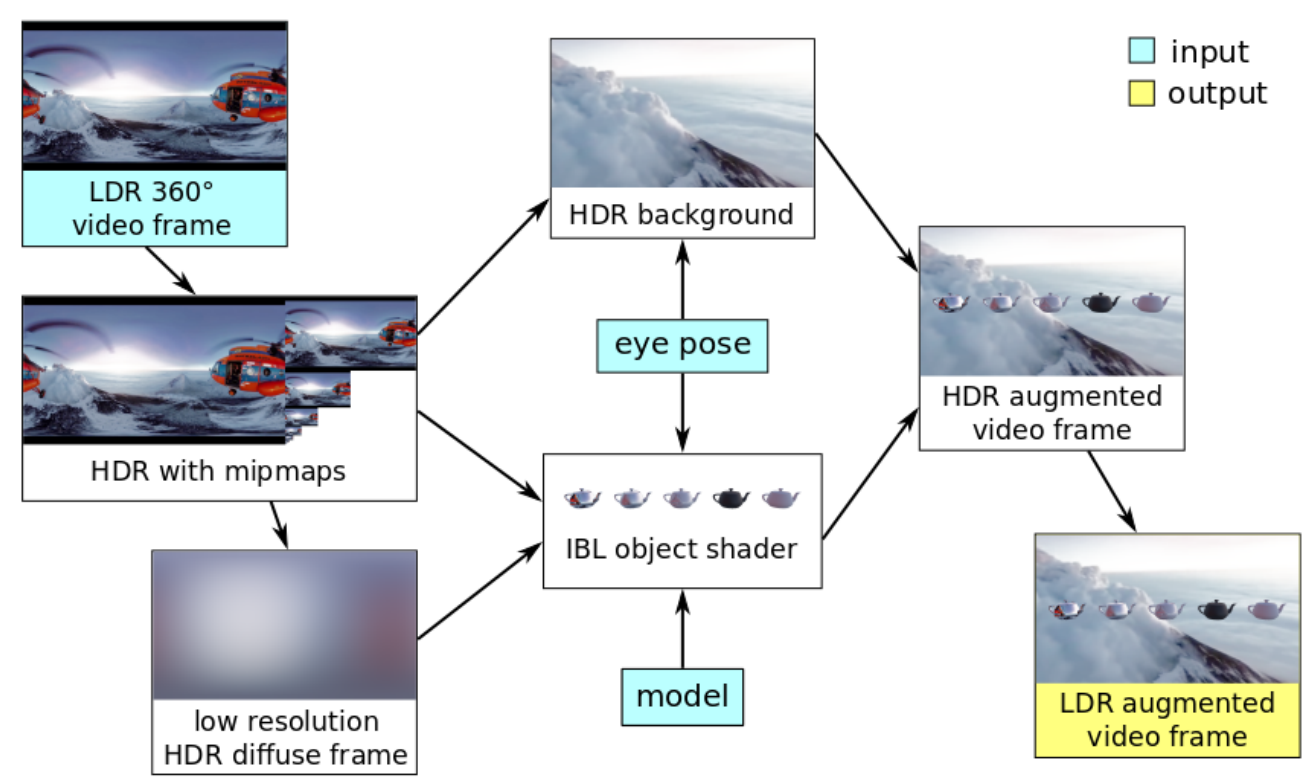

Figure 4.5: Diagram of solution pipeline showing the inputs and outputs of the various stages.

Each incoming video frame is first tonemapped from LDR to HDR using an appropriate algorithm. The HDR frame is mipmapped and the mipmapped result is treated as a set of filtered irradiance maps for this frame. An HDR diffuse radiance map is calculated from the most appropriate irradiance map according to performance and quality requirements.

As input framerate is expected to be lower than output framerate, these values may be used for several frames of real-time output.

Each output frame is constructed by combining a perspective view of the original LDR 360 video with a perspective rendering of the desired virtual objects according to the same procedure as detailed in section 4.1.3.

\section{Prototype Implementation}

A new prototype implementation was created by building on the previous prototype, and as such much of it does not need to be described. Implementation was mostly 
similar to that of section 4.1.3, with the addition of a shader stage converting the LDR input frame into an HDR texture. For this step a fragment shader was used to apply a simple inverse gamma transform with hardcoded parameters.

As previously, the prototype is implemented using $\mathrm{C}++$ and OpenGL 3.3. For model loading the Open Asset Import Library [46] is used, and for video loading the OpenCV [37] library is used.

\section{LDR-HDR Tonemapping}

The inverse tonemapping transform used can be described by a scaling factor determined according to the luminosity of the input at a given pixel position

$$
L_{s}=10 \cdot L_{i}^{10}+1.8
$$

where $L_{s}$ is the scaling factor, and $L_{i}$ is input luminosity. The form of this equation can be considered a compromise between the tonemapping operators used in [3] and [9], and the exact values used here were determined after experimenting with various inputs and operators. From these experiments it appears that many simple tonemapping operators would be acceptable, but the one described here was found to produce consistently good results across a wide variety of input lighting conditions with the fixed parameters declared above.

The scale factor is applied directly to the red, green and blue channels of the image as

$$
\left[R_{o}, G_{o}, B_{o}\right]=L_{s} \cdot\left[R_{i}, G_{i}, B_{i}\right] .
$$

For low-intensity values the conversion is linear, but input values nearing peak brightness will begin to be scaled exponentially. Interpreting input values on a scale from 0.0 to 1.0 , output values when multiplied by the scaling factor $L_{s}$ will be between 0.0 and 11.8 (see figure 4.6).

Input luminosity is determined from the red, green and blue input channels as

$$
L_{i}=0.3 \cdot R_{i}+0.59 \cdot G_{i}+0.11 \cdot B_{i},
$$

which matches well with brightness as perceived by humans. 


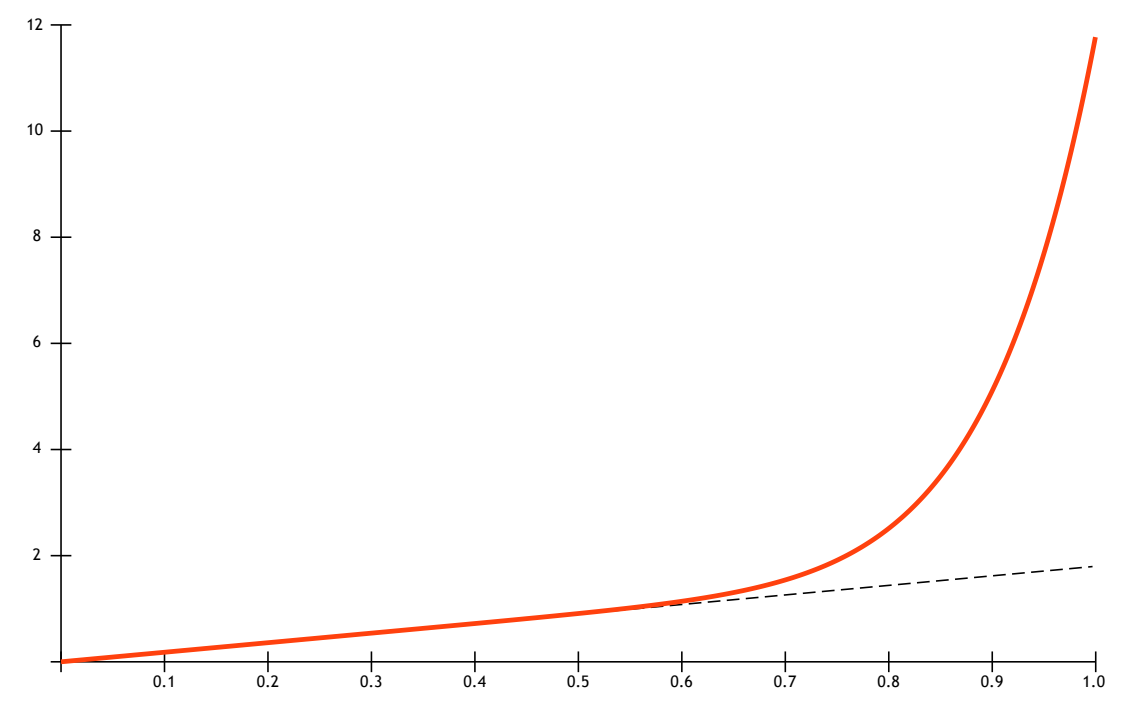

Figure 4.6: Plot of the inverse tone mapping operator used here. The red line corresponds to $y=\left(10 x^{10}+1.8\right) x$ and the dashed black line to $y=1.8 x$. Thus the operator here follows a linear relationship for most input pixels, but enhances the brightness of the brightest pixels exponentially.

The dynamic range of the output of this transform is not particularly great, but it was found to be sufficient for believable lighting and robust to a wide variety of real-world inputs. Determining the best tonemapping operator for use on 360 degree video for IBL could be an interesting topic for future work, but as finding an acceptable operator was not difficult further optimization was not sought.

\section{Final Pipeline}

With the input tonemapping operator as specified, the final implementation pipeline is as follows.

Virtual object models and input video are loaded on the fly, so on each rendering cycle if the desired model or video is not loaded the loading is performed. This is done using the external libraries mentioned previously, and optimization of this step is considered out of scope for this implementation. Once the model or video is loaded, this step can be disregarded. 


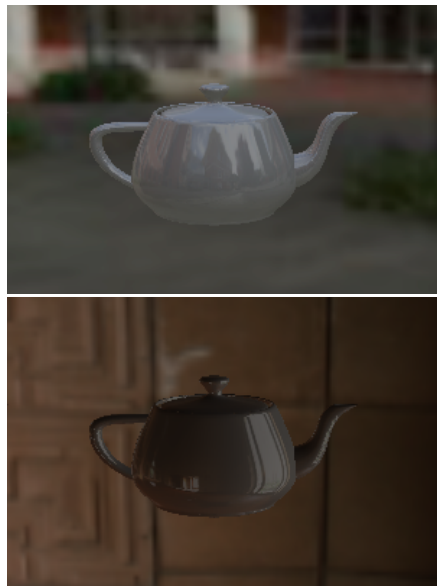

(a) LDR

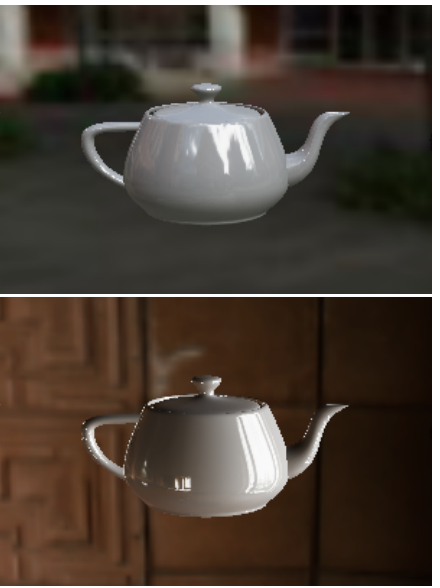

(b) tonemapped LDR

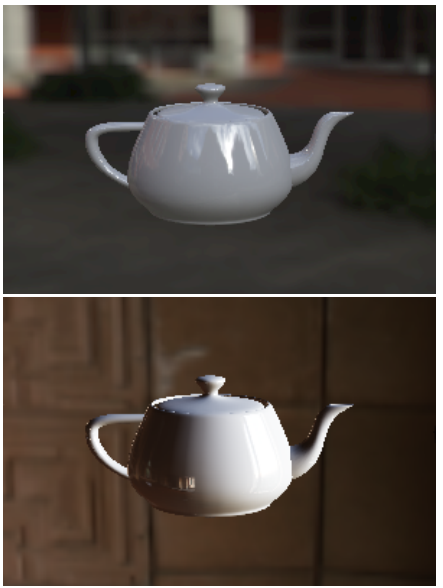

(c) HDR

Figure 4.7: Results of IBL performed directly using an LDR environment map (left), using an LDR-HDR tonemapped environment map (middle), and using an HDR environment map. The LDR-only result can be seen to be dull and unrealistic. The LDR-HDR tonemapped result can be incorrect for extreme cases such as that of bottom image, but still achieves a crisp, believable appearance.

Input video frames are stored in a pair of OpenGL textures with one being used for display, and the other being used to load the next video frame. According to the framerate of the video, when a new video frame is required the LDR input frame textures are swapped and the three stages of LDR-HDR tonemapping, HDR mipmapping, and diffuse radiance map generation are performed using the new one. The next video frame is decoded and loaded into the old texture in parallel as a background process.

For each rendering frame (of which there may be several per input video frame) a perspective view of the HDR 360 degree video is rendered to an HDR texture, and the current HDR mipmapped input frame and diffuse radiance map texture are used to render the virtual objects on top of this texture as described in section 4.1.3. The output is then tonemapped as previously while rendering to the display.

If output to HMD is desired, the viewer's head position and orientation are used as the current camera position and orientation when rendering, and the rendering 
step is performed twice, once for each virtual eye of the HMD, with appropriate tweaks to camera location according to relative eye positioning.

\section{Results}

The 360 degree video based real-time IBL prototype was tested on a variety of videos from the video sharing website YouTube [54]. Videos were chosen so as to provide as many different lighting conditions as possible for testing, and the videos were then used as input for the developed real-time IBL prototype, inserting virtual objects into a perspective view of the original scene. Some of the results can be seen in figures 4.8, 4.9, 4.10, 4.11 and 4.12.

The inverse tonemapping operator of equation 4.1 was found to give believable results matching the lighting of the input video in all tested cases. The parameters given in equation 4.1 were used in all cases without variation, and no other manual intervention was used. The proposed real-time IBL system is thus demonstrated to be both automated and robust. Every frame was rendered separately with no prior (or ongoing) analysis, and so there is theoretically no barrier to live streaming realtime use, although this was not directly implemented in the prototype.

Performance was worse than the non-video prototype, with the low end GeForce 730 rendering $1280 \times 720$ augmented video at a lowered $15-30 \mathrm{~Hz}(30-60 \mathrm{~ms}$ per frame), however a GeForce 690 was still able to render at around $90 \mathrm{~Hz}(11 \mathrm{~ms}$ per frame) and the GeForce 980 at around $500 \mathrm{~Hz}$ (2ms per frame). In addition, the 690 and 980 were both able to render comfortably at over $60 \mathrm{~Hz}$ to the Oculus Rift DK2 HMD with stereo output of $1182 \times 1464$ resolution per eye. As the added tonemapping operation is computationally trivial the reduction in performance relative to the first prototype is likely caused by either video decoding overhead or memory bandwidth limitations.

The results of this work have been accepted for peer-reviewed publication under the title "Real-Time Image Based Lighting for 360 Degree Panoramic Video" by Thomas Iorns and Taehyun Rhee [21]. 

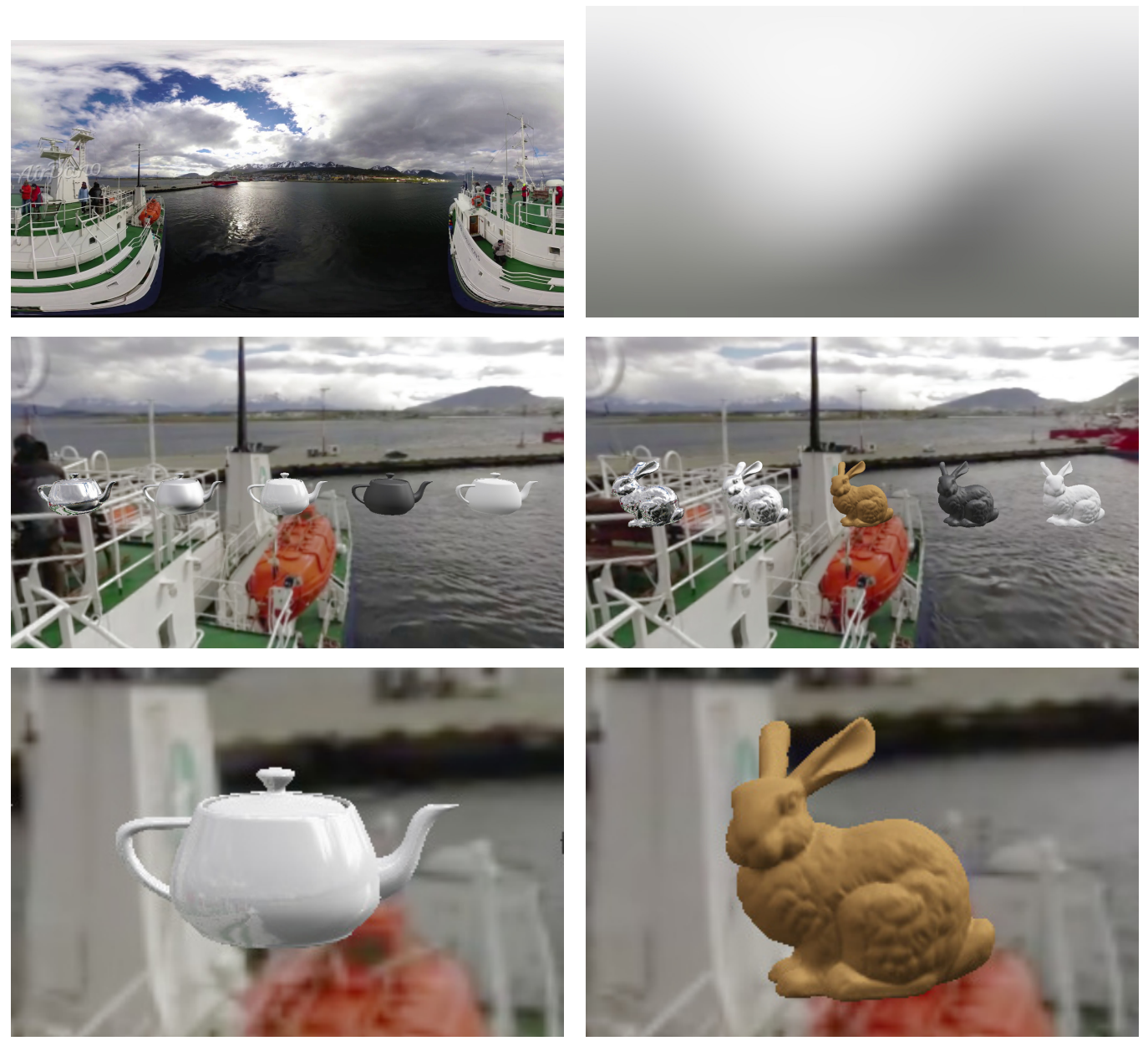

Figure 4.8: 360 video frame (top left), generated diffuse radiance map (top right), and a rendered view augmented with virtual teapots and bunnies (middle and bottom). 

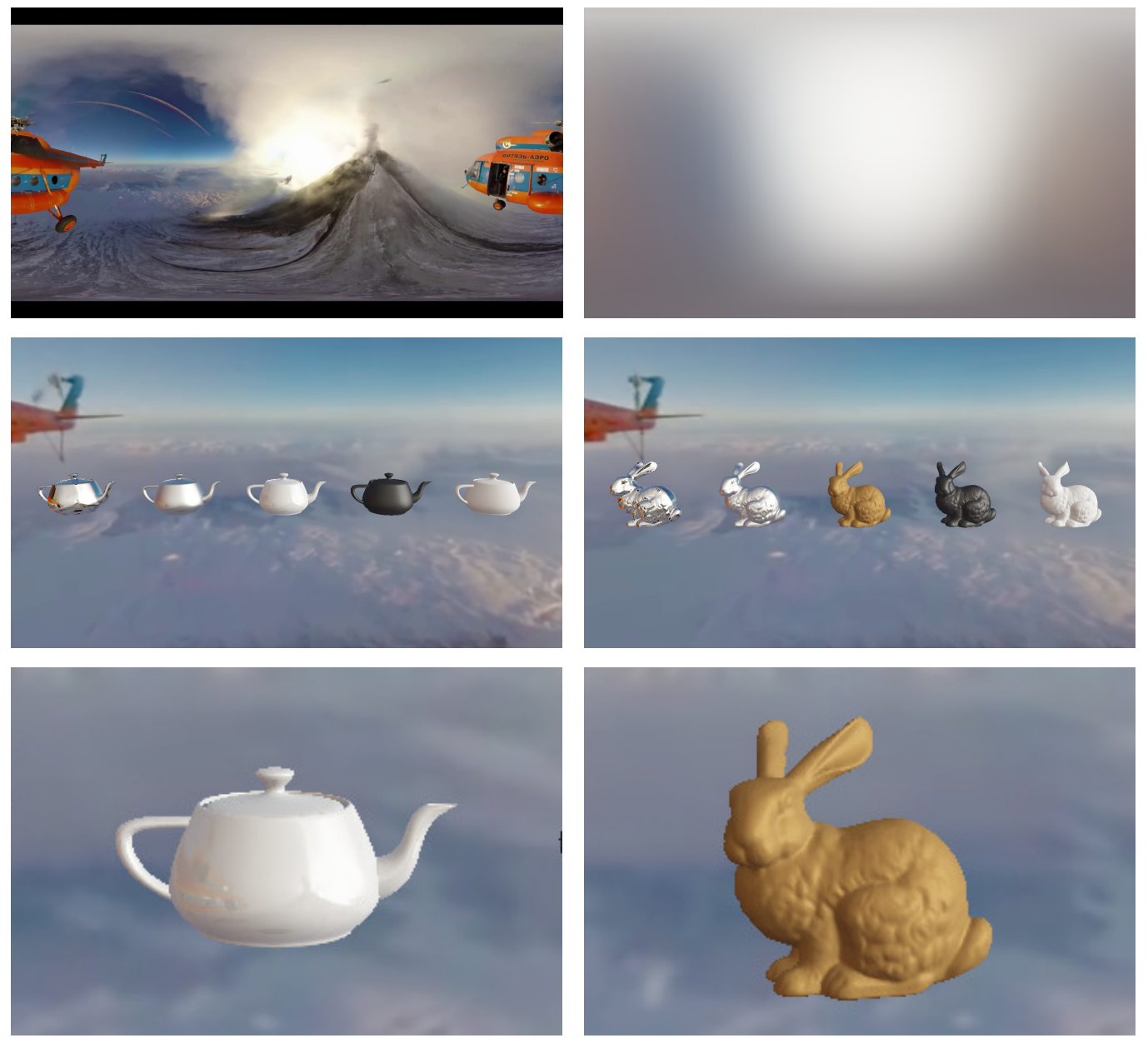

Figure 4.9: 360 video frame (top left), generated diffuse radiance map (top right), and a rendered view augmented with virtual teapots and bunnies (middle and bottom). 

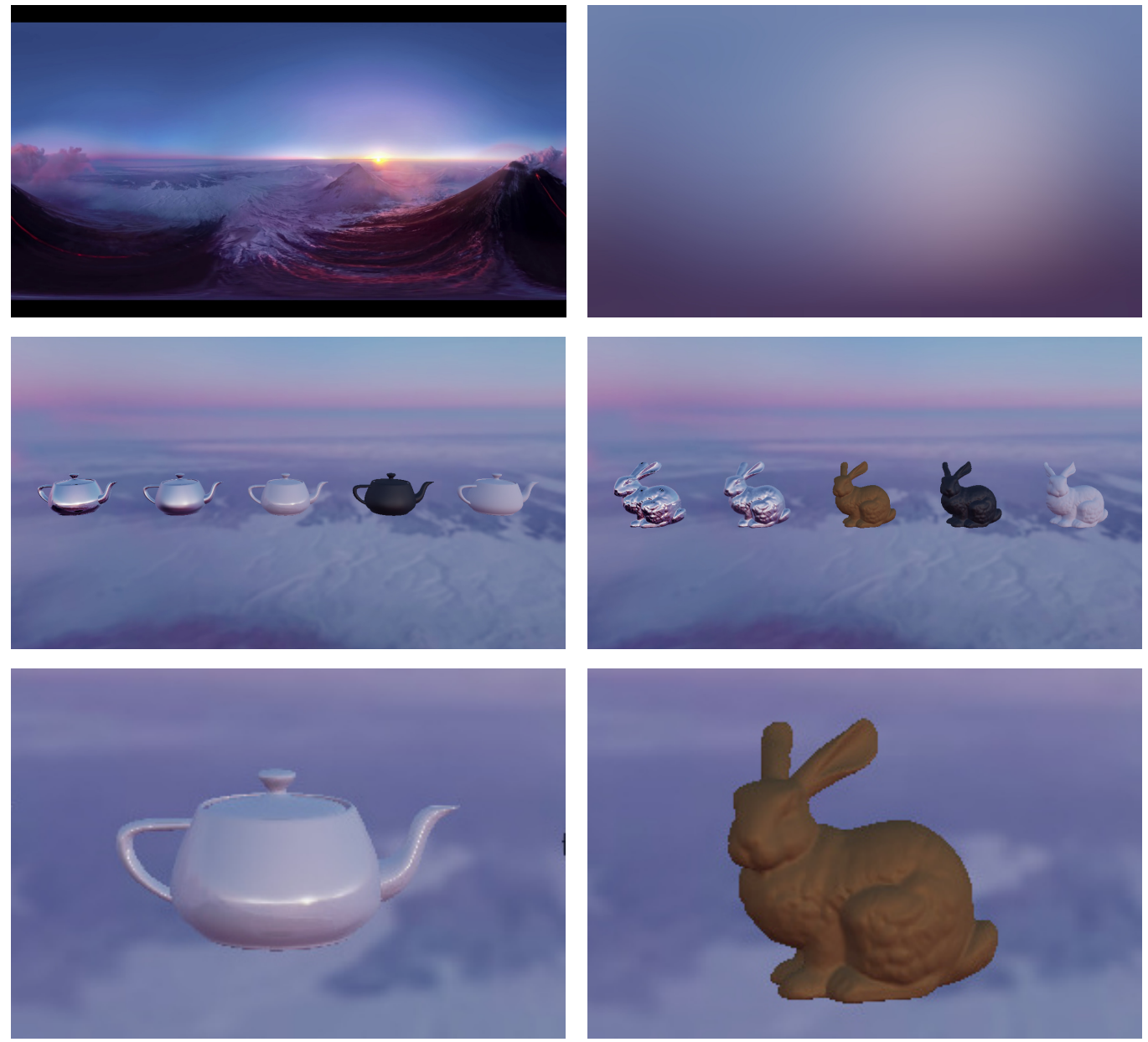

Figure 4.10: 360 video frame (top left), generated diffuse radiance map (top right), and a rendered view augmented with virtual teapots and bunnies (middle and bottom). 

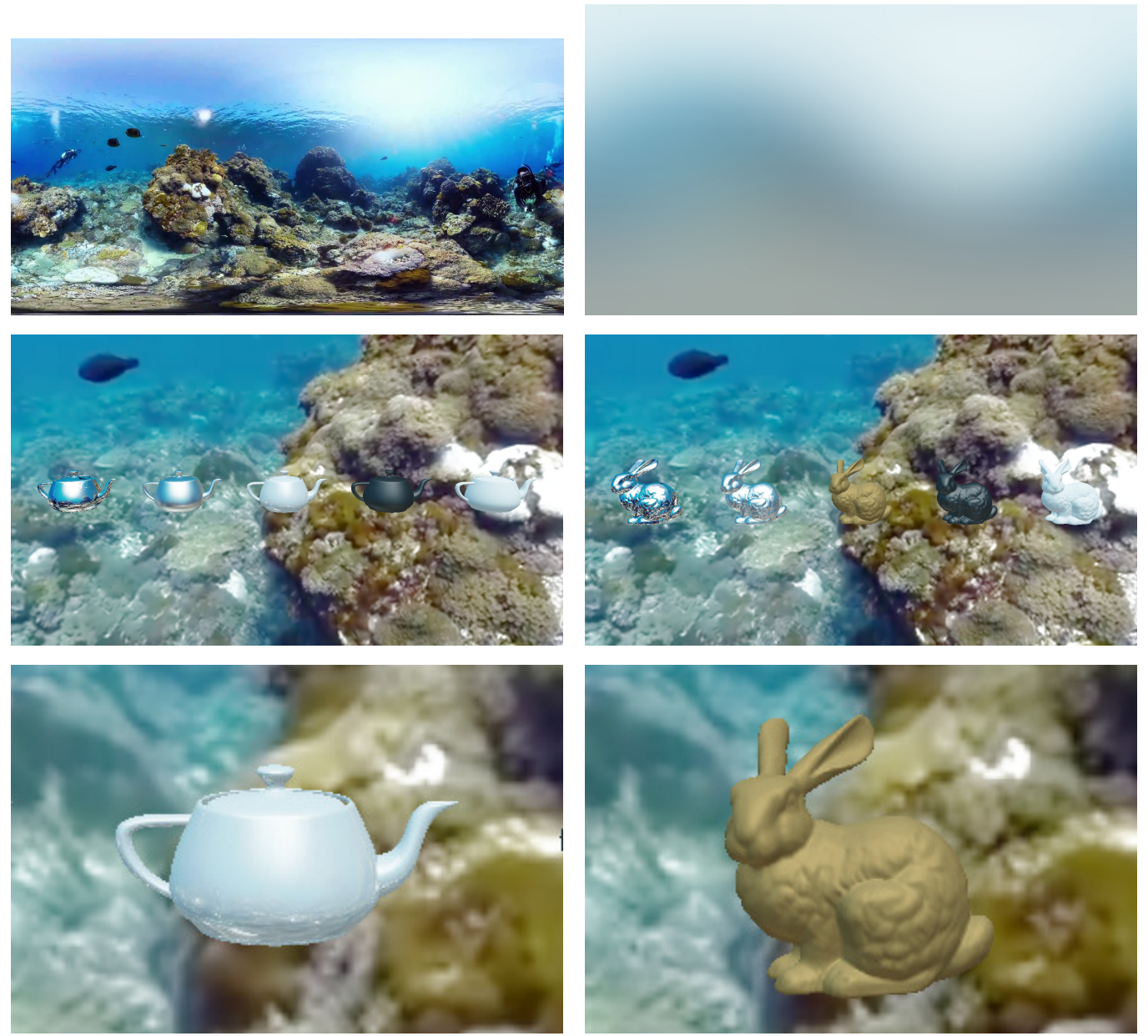

Figure 4.11: 360 video frame (top left), generated diffuse radiance map (top right), and a rendered view augmented with virtual teapots and bunnies (middle and bottom). 


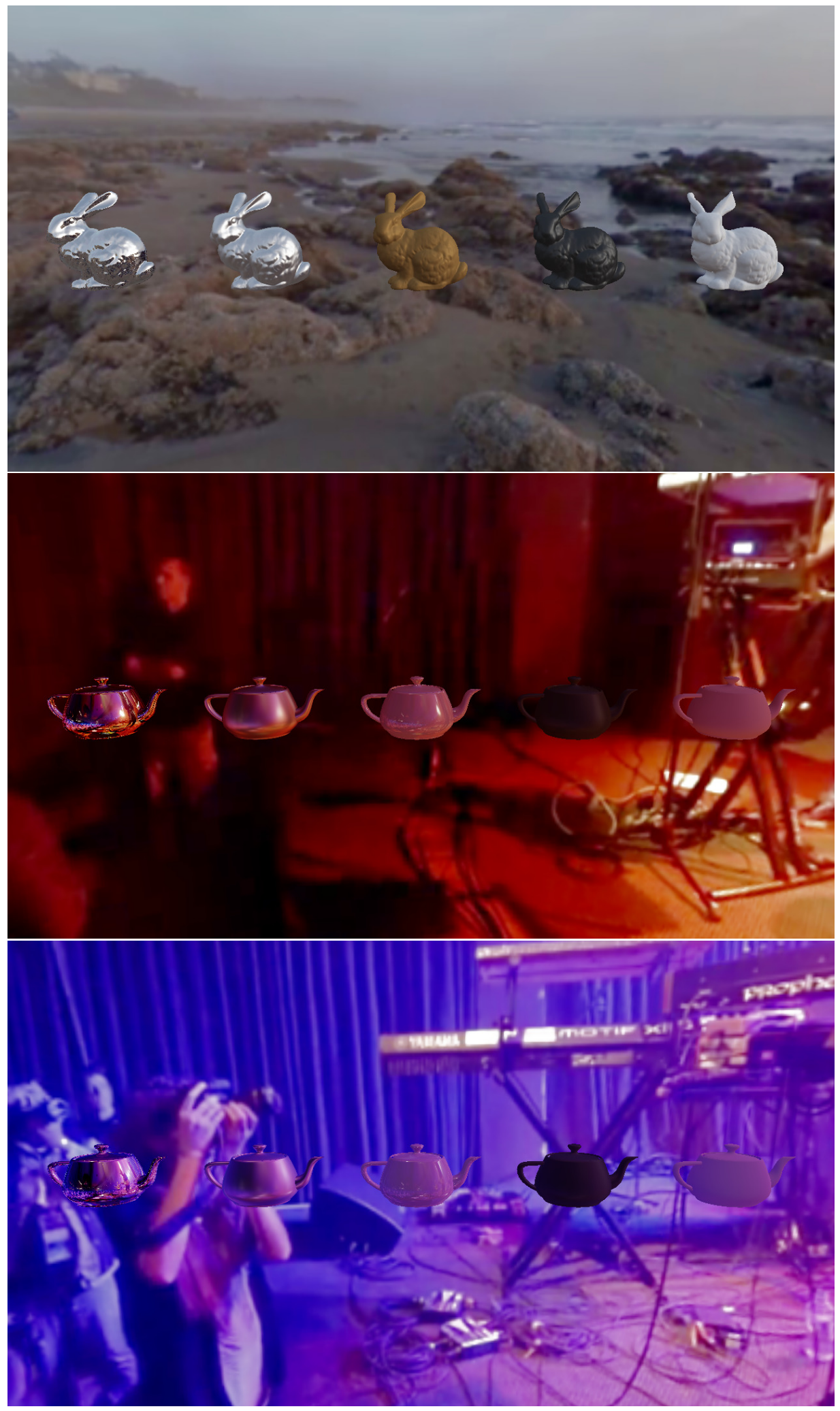

Figure 4.12: Results in scenes with various types of lighting. Objects were lit believably in all tested cases (including many not shown here). 


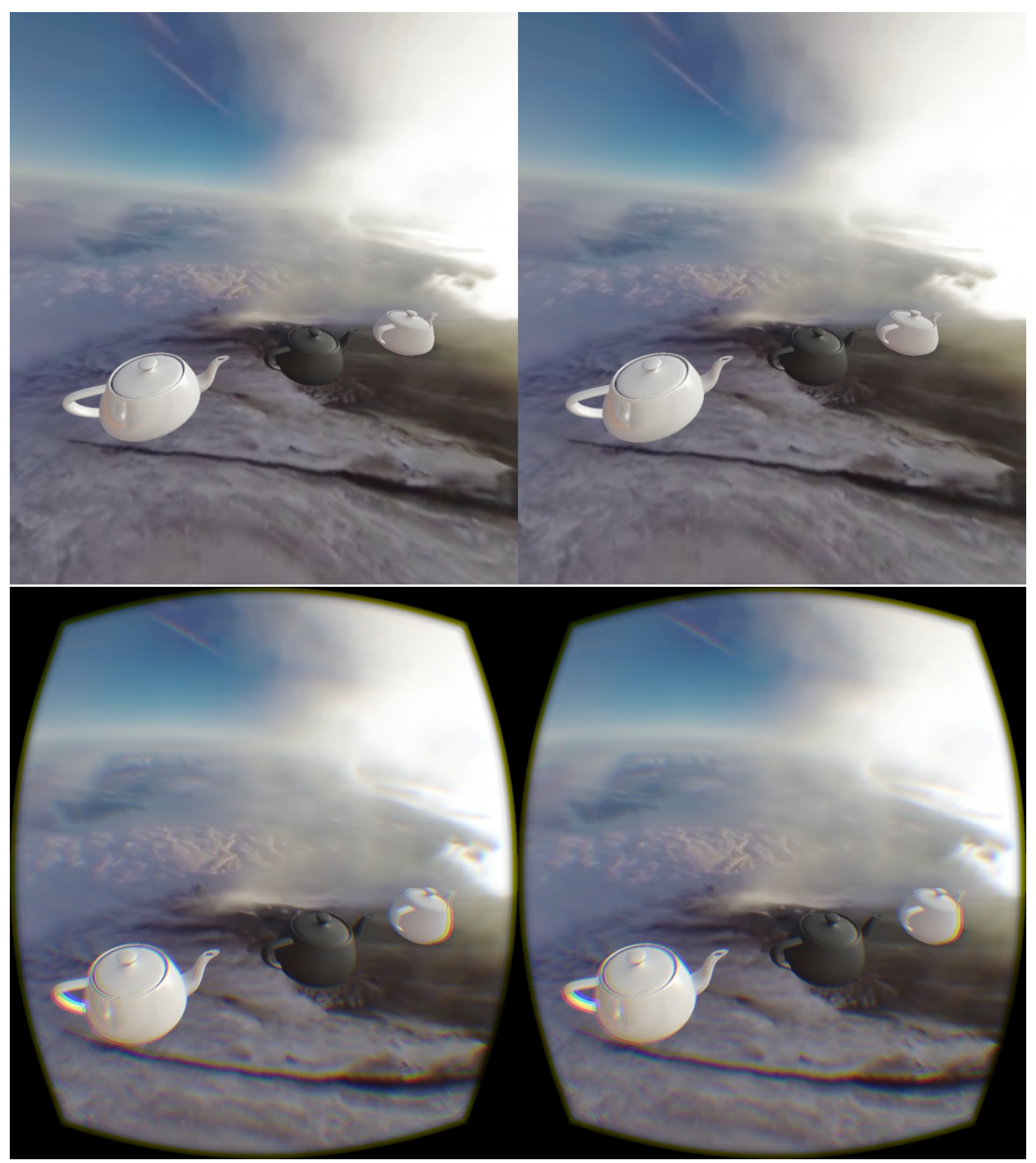

Figure 4.13: Output as rendered for the Oculus Rift DK2 HMD (top), and the actual output displayed on the interior screen of the device (bottom). Lenses in the device cause each eye of the viewer to see one of the displayed images in a wide field of view. The images displayed on the interior screen of the HMD have been warped and filtered chromatically (an automatic process performed by the device driver) to account for the warping and chromatic abberation of the lenses used in the device. 


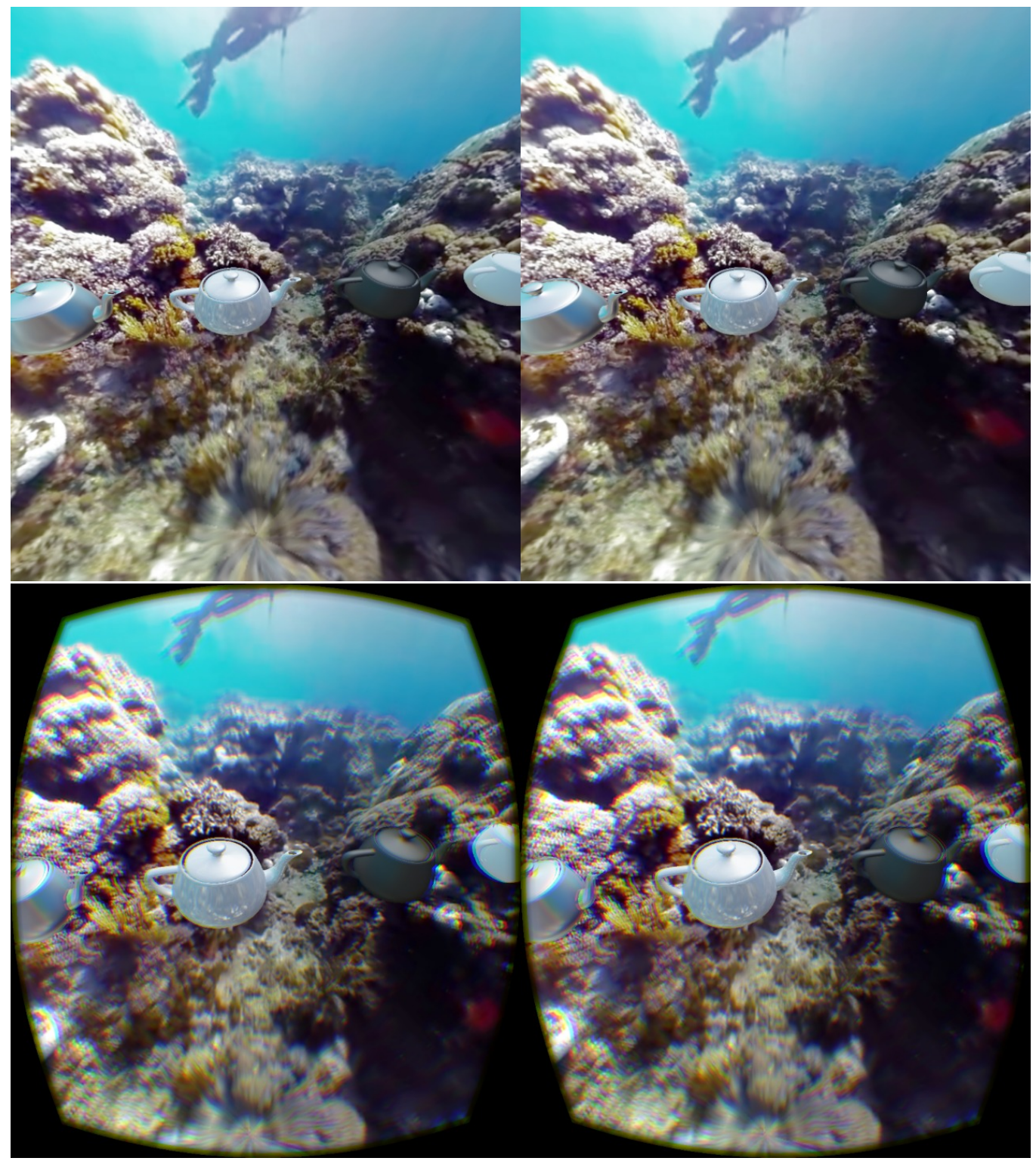

Figure 4.14: Output as rendered for the Oculus Rift DK2 HMD (top), and the actual output displayed on the interior screen of the device (bottom). Lenses in the device cause each eye of the viewer to see one of the displayed images in a wide field of view. The images displayed on the interior screen of the HMD have been warped and filtered chromatically (an automatic process performed by the device driver) to account for the warping and chromatic abberation of the lenses used in the device. 


\section{Chapter 5}

\section{Applications}

So as to demonstrate the practical applicability of the solution described in chapter 4 , several real-world use cases were explored. The system was found to work well in actual usage, demonstrating its robustness and practicality outside of the controlled testing conditions used in development.

\section{Implementation in Unity3D}

As the system developed for real-time IBL using 360 degree video works well in the prototype implementation we decided to test its capability for application in the popular game design framework Unity3D [47]. This test concerns both the adaptability of the system to a different framework than the one it was designed for, and its performance under a constraint where the exact rendering pipeline cannot easily be optimized.

Aside from its popularity, one of the benefits of the Unity3D framework is that it supports the Oculus Rift series of HMDs automatically, allowing us to determine directly whether HMD rendering can be supported at the necessary framerate. As it also supports video textures and user-programmable material shaders, it should be possible to apply the developed system in this framework. 


\section{Technical Challenges}

For this application to be considered successful it should demonstrate an implementation in the Unity3D framework of the real-time IBL technique described in chapter 4 , rendering $\operatorname{LDR} 360^{\circ}$ video to $\mathrm{HMD}$ with virtual objects added to the depicted scene and lit by the provided $360^{\circ}$ video. HMD framerate must be smooth and object lighting should be believable.

One concern of trying to adapt the previous impementation to Unity3D is that the shader languages are not directly compatible. Previously OpenGL's GLSL shader language was used to program the real-time IBL computation, but Unity3D uses a different system so the previous shader code will have to be converted to apply correctly. This may or may not be difficult, depending on whether the previous implementation depended on OpenGL-specific data structures, functions or programming patterns.

Another concern is that the previously used method of chaining together multiple rendering passes, with different shaders rendering to textures which are then used by successive shaders, does not appear easily implemented in Unity3D. As a game design framework supporting many different runtime environments it primarily has a fixed set of prescribed shader pipelines, none of which appear at first glance to correspond to the pipeline used in the previous $\mathrm{C}++$ and OpenGL implementation.

A third concern is that the HDR texture support of Unity3D will not be sufficient. Although it does support HDR lighting internally, the mechanism for doing so is not easily overridden and it may turn out to be unsuitable for this application.

\section{Implementation}

The Unity3D implementation can be divided into several components which allow the system for real-time IBL from 360 degree video to be realized.

\section{Video Display}

Because Unity3D already has a complete rendering pipeline set up to generate perspective views of a 3D scene appropriate for HMD, the easiest way to display the 


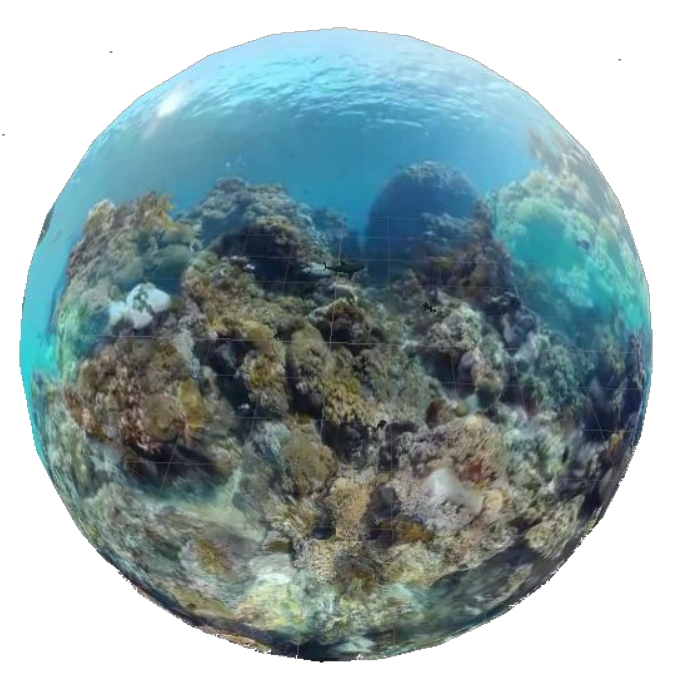

Figure 5.1: Sphere with underwater 360 video frame mapped to the inside. When a view is rendered (via HMD or otherwise) from the centre of this sphere, the video appears as an encompassing environment. Placing the environment at a fixed distance from the viewer in this manner was not observed to adversely effect immersion.

input 360 degree video is to map it onto the inside of a sphere and model it as part of the default Unity3D scene. The viewer's position can be set to the center of this sphere, with head and eye positions relative to this center point. In this way the standard 3D rendering path of the Unity3D platform causes the viewer to be immersed in the video. The only programming necessary for the implementation of this step consisted of a small material shader program for the sphere which inverted its surface normals (so that the video was displayed on its interior, instead of the exterior as would otherwise be usual), and a small script to start the video playing on scene load. Unity3D handled updates of the sphere's texture according to the expected video framerate automatically.

\section{Inverse Tonemapping and Mipmapping}

The inverse tonemapping step was slightly more convoluted to implement. In the end it was most convenient to construct a plane inside the scene, outside of the sphere displaying the 360 video and thus invisible to the viewer. An orthographic camera 


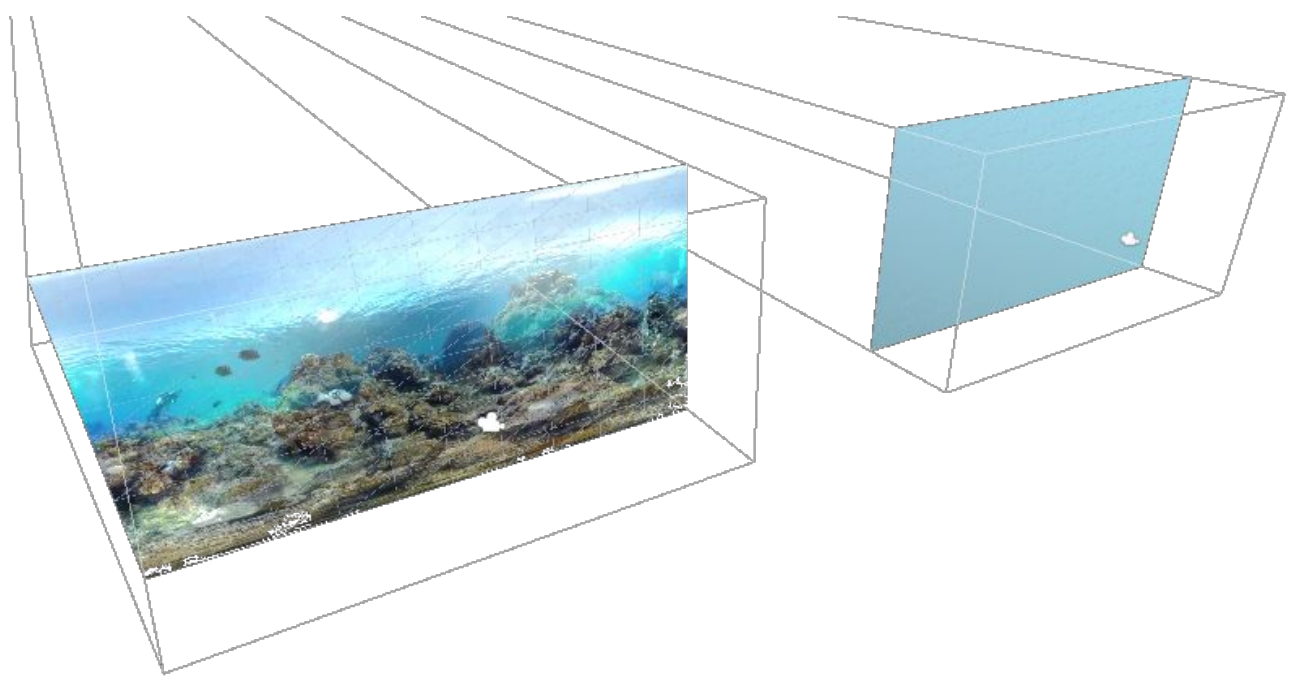

Figure 5.2: Diffuse map generation pipeline in Unity3D, implemented as a pair of orthographic cameras viewing rectangular surfaces. Material shaders assigned to the surfaces perform tonemapping and diffuse illumination transforms, with each result captured by the corresponding camera. Here an underwater scene is tonemapped and its (rather bland) diffuse radiance map generated.

was arranged so that its view precisely coincided with the plane (see figure 5.2). The inverse tonemapping operation was set up as a material shader taking the 360 video texture as input and outputing material colour in HDR according to surface position. The orthographic camera was configured to render to a texture of an adequate size (in this case $1024 \times 512$ ), and the built-in Unity3D option for HDR rendering was enabled for the camera. This resulted in an automatically-updated HDR version of the current input video frame. As an option to automatically generate mipmaps for this render texture was available, it was also enabled, and thus the output of this orthographic camera could be used as an HDR texture representing the mipmapfiltered irradiance map as desired. 


\section{Diffuse Radiance Map Generation}

In a similar manner to that of inverse tonemapping, another plane was constructed with a material shader designed to calculate a diffuse radiance map. The code for this material shader was equivalent to that of the OpenGL implementation, and a direct numerical computation of equation 2.2. Another orthographic camera and render texture were assigned to record the output of this shader. Essentially this technique is equivalent to that of the prototype implementation in section 4.1.3, but instead of assigning shader programs directly the virtual plane and camera were set up as a part of the main scene. The presence of a physical metaphor for the shader pipeline had the unexpected benefit of making shader inputs and outputs easier to manage, but it relies on Unity3D correctly handling writing to and reading from the render textures in the correct order.

\section{IBL Application}

The render-texture outputs of the two orthographic cameras were able to be used as inputs to a material shader implementing IBL. The associated material could then be applied to any object in the scene as desired. Unity3D allowed variable inputs to the shader and these were used to set material properties such as diffuse colour, specular colour, and surface roughness. Diffuse and specular textures were also easily enabled as an alternative to flat material colour. The generated irradiance and diffuse radiance maps were also assigned as inputs to this material shader, using Unity3D's built-in HDR texture format.

The constructed material shader was analogous to the one used in section 4.1.3, and in fact thanks to the general similarity of graphics processing methods, most of the original GLSL shader code was able to be directly converted to Unity3D's preferred shader language by simply renaming data structures and function calls to their native equivalents. Program structure was mostly unchanged. 


\section{Final Output}

Having completed development of these components, adding virtual objects lit using IBL to the scene was as simple as inserting a 3D model, assigning the IBL shader, and choosing material properties for it. The Unity3D platform then took care of rendering, display, and camera positioning automatically. Once set up, to change the environment requires only assigning the new video to the display sphere and tonemapping plane, after which IBL will automatically be applied in real-time to any objects with the correct material assigned, and the results displayed via HMD.

\section{Results}

The Unity3D implementation was tested with the same videos as were used in section 4.2, and performance was found to be comparable. Rendering to the Oculus Rift DK2 HMD was tested using an Nvidia GeForce 980M graphics card and found to run smoothly at $60 \mathrm{~Hz}$.

The primary benefits to using Unity3D were in the convenience of the included features such as HMD support. Rendering to HMD simply involved selecting the appropriate Unity3D option and running the program as normal. Although they were not used here, the built-in support for extras such as normal maps means that the IBL implementation could likely be easily extended within this system.

The lack of dynamic range in the built-in HDR lighting support turned out not to be a problem, partially because the dynamic range of the inverse tonemapping operator used in the real-time IBL system was also low, and partially because input 360 degree videos were typically already fairly balanced in terms of exposure settings.

Overall the implementation was successful, demonstrating the adaptability of the proposed real-time IBL system between frameworks.

\section{Interactive 4D Home Entertainment System Demo}

One of the benefits of the developed real-time IBL system is that it should allow high-quality AR and MR applications in the case where 360 video is available. To 
test and to showcase this application, a piece of demo software was developed as a collaborative project between four different universities. The goal of the demo was to provide an immersive and interactive entertainment experience based on 360 degree video, and to showcase the work of students collaborating on the $\mathrm{HDI}^{2} 4 \mathrm{D}$ project [15] to develop the next generation of $4 \mathrm{D}$ home entertainment.

\section{Technical Challenges}

To be considered successful, this application should provide an entertaining interactive 4D home entertainment system demo augmenting 360 degree video with virtual objects so that they appear part of the scene depicted in the original video. Virtual objects must be interactive and the overall experience should increase immersion in the depicted environment.

One major concern when implementing this demo was that thus far shadowing effects (other than those captured by the original 360 degree video) had not been taken into account. This meant that the demo must include objects that either fly or float, and that are not expected to be frequently in close proximity to one another.

The second main concern was that the project was a collaboration between four different universities spread across two countries. It was not known before beginning how easy or difficult it would be to implement this IBL system as part of a real-world project involving multiple collaborators working independently.

\section{Implementation}

The MR demo was developed in the Unity3D platform, building on the real-time IBL implementation described in section 5.1. To satisfy the requirements of discrete floating or flying objects, an underwater SCUBA diving video was chosen. An auxiliary benefit to using this type of video is that the slow movement and generated sensation of floating appear unlikely to induce simulator sickness [27] which can be a problem for people using an HMD for the first time.

To augment the underwater scene, some virtual fish and a shark were added. These fish were assigned the simple behaviour of swimming in circles around the 
viewer's position. The IBL material shader described in section 5.1.2 was assigned to the fish and suitable diffuse and specular reflection proportions were assigned. As glossy specular reflection was not needed to model the reflectance properties of fish scales, it was disabled. Because the video was LDR and focused on the underwater environment, the lighting from above was often saturated to the point at which it lost colour, so to compensate a slight blue tint was added to the diffuse colour of the material on all objects to match with objects in the environment. After this minor tweak, the appearance of virtual objects was similar to that of depicted real objects in all scenes of the video, which included a variety of lighting conditions ranging from open sunlight to an enclosed underwater tunnel.

For interactivity a Leap Motion [20] controller was used. This controller tracks the position and orientation of the viewer's real-life hands and fingers, allowing virtual hands to be inserted into the scene corresponding to where the viewer is placing their hands in real life. Collision detection could then be performed between these virtual hands and the virtual fish placed in the scene, allowing the user to reach out and touch them by physically doing so with their hands (see figure 5.4a). The virtual hands were lit using the IBL material shader developed previously, contributing to believability and immersion. Additionally so as to provide a physical sensation of having touched something when interacting with the fish, a vibratory haptic feedback device was used, stimulating the viewer's fingertips with vibration in accordance with determined points of contact.

\section{Results}

The resulting demo software was a successful integration of its various components. The IBL lighting worked well and for the most part did not interfere with the work done by the other students. One particular point of success was the application of the IBL shader to the virtual hand model integrated by another student. This was as simple as changing the material shader for the hand model being used to the one developed for real-time IBL, retaining the diffuse hand texture. An objective evaluation of the result was not performed, however subjective feedback indicated that the hand without IBL looked somewhat fake and this attracted people's attention 


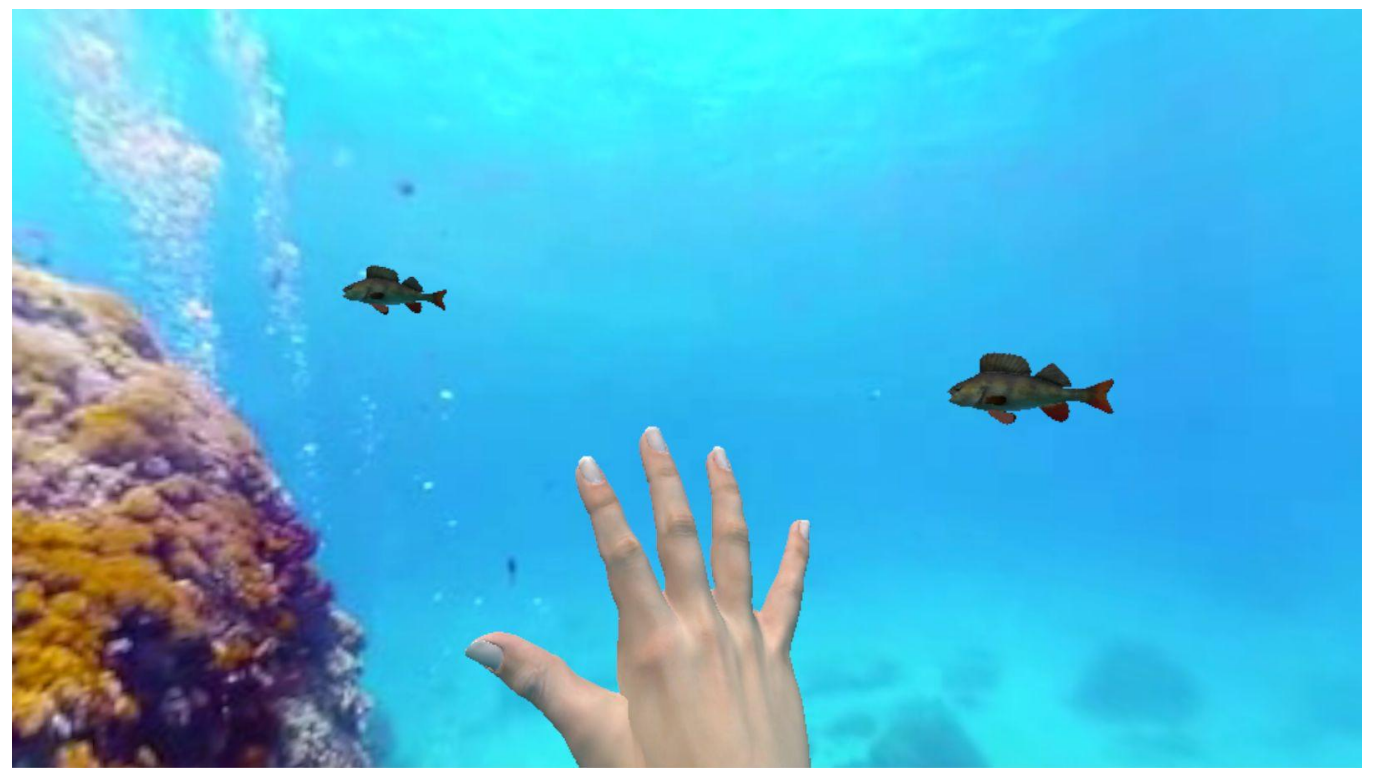

Figure 5.3: Virtual environment of underwater $360^{\circ}$ video, augmented with a virtual hand mimicing the real-life position of the user's hand, and some virtual fish swimming about.

to it as something that stood out from the scene. Once the IBL material was applied to the hand, viewers seemed to spend less time concentrating on the hand, and more time using it as a proxy for their real-life hand and interacting with the fish. This purely anecdotal evidence suggests that having IBL applied to the virtual hand may directly increase immersivity, which could be an interesting direction for further research.

The biggest problem with the resulting demo was that Unity3D would not allow the use of a video texture of higher resolution than $1280 \times 720$. The low resolution input video turned out to be the main factor reducing believability of the MR environment. Specifically when virtual objects were rendered at high resolution they stood out against the low-resolution backdrop, making them easy to separate. This can be considered as partially because a technique such as that of [11] was not used, the fish were simply rendered on top of a perspective view of the scene. An interesting avenue for further research might be to examine methods of matching the 


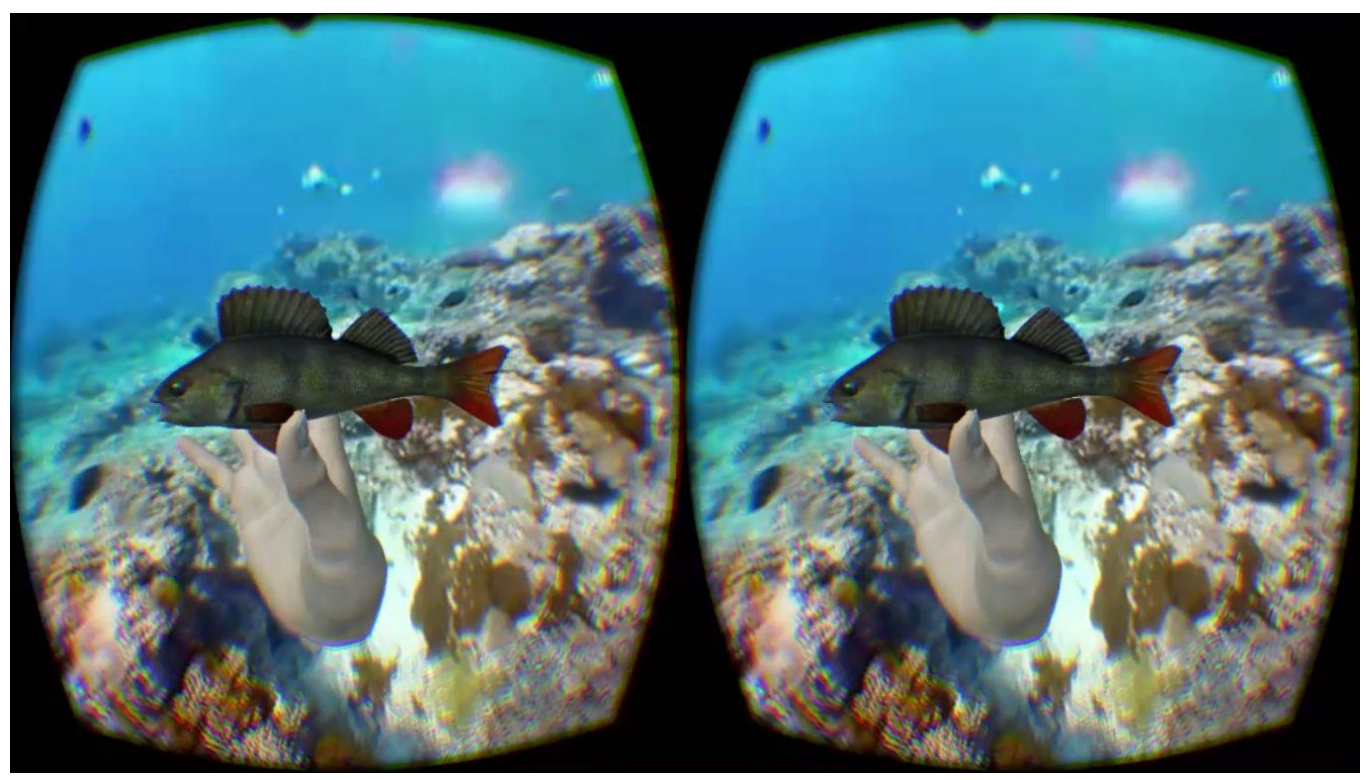

(a) Catching virtual fish using a real-life hand counterpart.

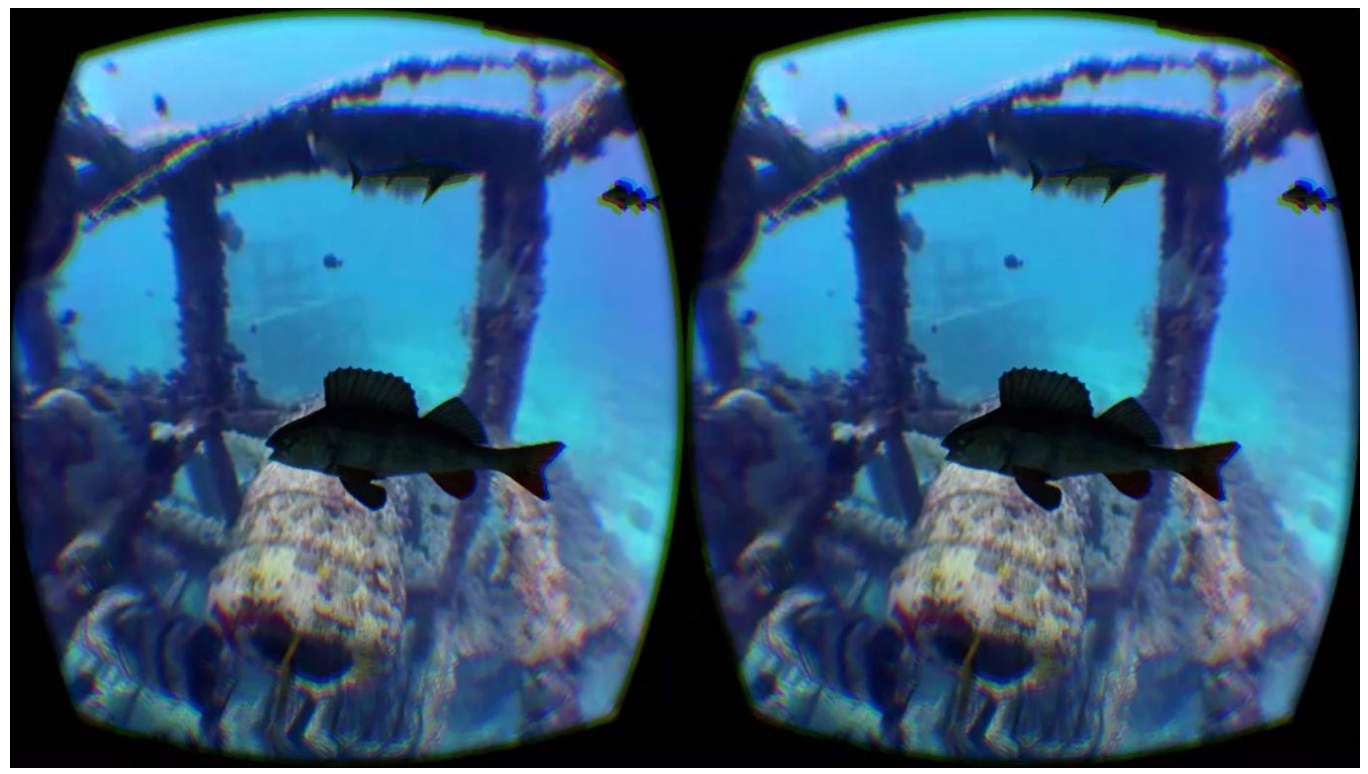

(b) Virtual fish swimming in shadow.

Figure 5.4: HMD output for MR underwater scuba diving demo based on $360^{\circ}$ video. 
resolution of the rendered objects with the video backdrop. Reducing the rendering resolution of the fish was experimented with and this was found to somewhat ameliorate the effects of the resolution mismatch, but not entirely. A more effective measure could be to somehow render the virtual fish directly into the background video frame, and then display this frame to the viewer. In this situation the resolution of the virtual fish would be identical to that of the video, also taking into account the warping of detail associated with the standard latlong storage format for 360 degree video.

On the whole the integration was a success, showing that real-time IBL can be effectively used for MR applications involving 360 degree panoramic video. The previously developed real-time IBL system was also shown to be able to interface well with components created independently by multiple collaborators.

\section{Acknowledgements}

This work was done in collaboration with Kiran Nassim from Ewha Womans University, Jaedong Lee from Korea University, and Joshua Chen from the University of Canterbury as part of the $\mathrm{HDI}^{2} 4 \mathrm{D}$ project [15]. The 360 degree video used as backdrop was created by David Hsieh [18] and made available for general viewing on YouTube [54]. The virtual fish model used was created and made available for public download under the Creative Commons Zero license on Blendswap [31] by the user holmen [17]. The virtual shark model was created by Mark Loftesnes [32] and also made available for public download under the Creative Commons AttributionNoncommercial license on Blendswap [31].

\section{IBL using Non-Panoramic Legacy Photographs}

The success of the real-time IBL system for lighting of objects for MR purposes led to the question of whether inputs other than 360 degree video or envrionment maps could be used. One such real-life input that would be convenient to make use of is that of standard non-panoramic LDR digital photographs. The ubiquitous 


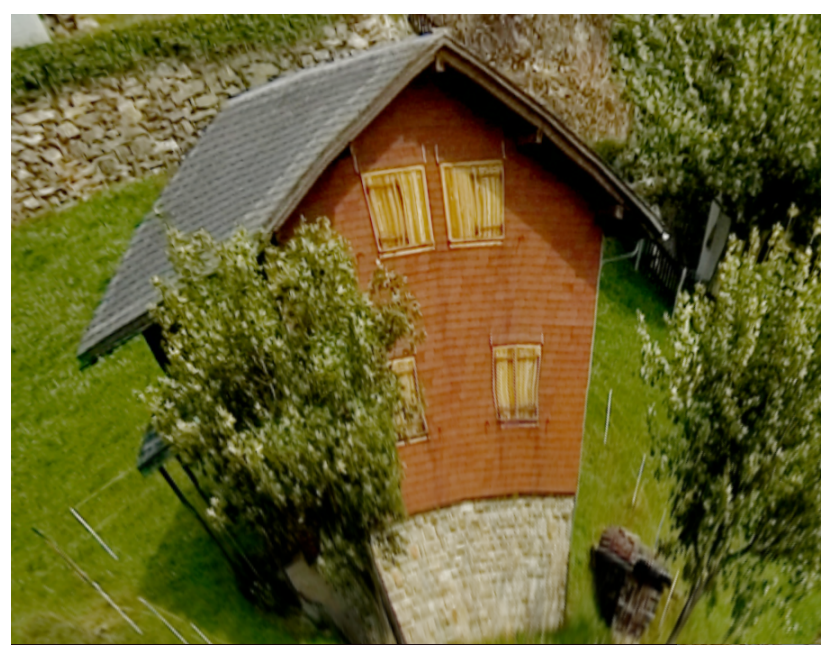

Figure 5.5: Warping artifact caused by stretching a standard photograph onto a sphere. The house's walls were originally parallel. When viewed via HMD, the scale of the house is also obviously incorrect.

availability of standard photographs as well as the personal connection to one's own photographs means their use in an MR scenario could be widely applicable.

As LDR to HDR tonemapping has been shown to be viable for IBL, the main problem to be addressed in order to use standard photographs for lighting is that of expanding or extrapolating the photograph to cover the entire sphere of the surroundings while still maintaining the appearance of important details in the depicted scene. Simply stretching the photograph will both cause details to be enlarged abnormally and cause warping artifacts as a near-planar projection is spread across the surface of a sphere (see figure 5.5). In addition, the edges of the photograph are unlikely to match up, leading to a noticable vertical line in reflections where the edges of a stretched photo would meet, and/or a noticable discontinuity in the background when viewed via HMD.

The solution here was explored in collaboration with Kurt Ma and Andrew Chalmers from Victoria University of Wellington and uses the technique of seam carving [5] as a preprocessing step to expand the photo so that it fills the entire sphere. Seam carving can be used to expand photographs while preserving important details, and 
as such may be ideal for this use case.

\section{Technical Challenges}

In this application the goal is to use only a standard non-panoramic LDR digital photograph as input, generating a believable irradiance map corresponding to the photograph and using it to light virtual objects in real-time with IBL. Ideally when using an HMD the viewer will be immersed in a scene similar to that represented in the photograph, and the virtual objects will appear similar to real objects depicted in the photograph.

The primary implementation concern is that the photograph may lack much of the information necessary to perform IBL directly. Most importantly, if the photograph does not include the primary light source of the scene in its field of view, it will be difficult to tell where in the environment this light source should be located and how it should contribute to IBL. For this reason we have restricted the scope of this experimental application to photographs including the primary light source, such as sunsets, sunrises, or overcast scenes.

Secondary to this is the problem of ensuring that discontinuities are minimal in the generated irradiance map. If it is constructed directly by expanding the original photograph using seam carving then there is no guarantee that the edges will match up.

\section{Implementation}

The developed system solution first uses a specially-programmed seam carving variant [33] to expand the photograph to fill the entire 360 degrees horizontally and up to 180 degrees vertically. This variant begins by locating the primary light source in the scene, and then performs the horizontal expansive seam carving algorithm so as to maintain the position of the light source in the final scene. A similar procedure is then performed for the vertical expansion.

As the top and bottom portions of images were often relatively uniform, representing either sky or ground, this second expansion step could often be performed 
to fill only the central 90 degree horizontal strip of the full sphere, and a simpler extrapolative procedure used to fill the sky and ground. Doing this was found to have little effect on the final IBL and viewing results, while also minimizing spherical warping.

Once a fully expanded photograph was obtained, the properties of the image at each horizontal edge were analyzed and matched to each other using an automated procedure. The image was then lienarly warped so as to minimize discontinuities between these edges which were then blended together to further remove any obvious edge discontinuity. The result is a fully-expanded 360-degree image which can then be used for IBL using the system described in chapter 4.

The expanded photograph was displayed as in the Unity3D implementation of sections 5.1 and 5.2 on the inside of a sphere with the viewer's position at the center. A cylindrical mapping might be a better transform for reducing warping relative to the original photograph but the spherical mapping was chosen so as to easily fill the entire possible viewport when viewed via HMD. In the case where the photograph is only expanded to within 45 degrees of the horizon, there is little difference in any case.

With this done virtual objects were rendered into the scene as in the previously described procedure using 360 degree video (see figure 5.6). Cases of displaying the original photograph on a flat plane, displaying an expanded version taking up approximately a hemisphere, and displaying the full spherical version were all tested. The full spherical version was found to be the most immersive, but also the most prone to artifacts resulting from the spherical warping and also from the extensive use of seam carving to expand the photograph.

On the whole the result of this application was determined to be somewhat successful and the experience immersive and entertaining, however the drawbacks of requiring the light source to be present in the photograph as well as the occasional warping artifacts caused by the spherical mapping and seam carving meant that it could only be applied effectively to a small subset of existing digital photographs.

This work has been accepted for peer-reviewed publication as "Synthesizing Radiance Maps from Legacy Outdoor Photographs for Real-time IBL on HMDs" by 


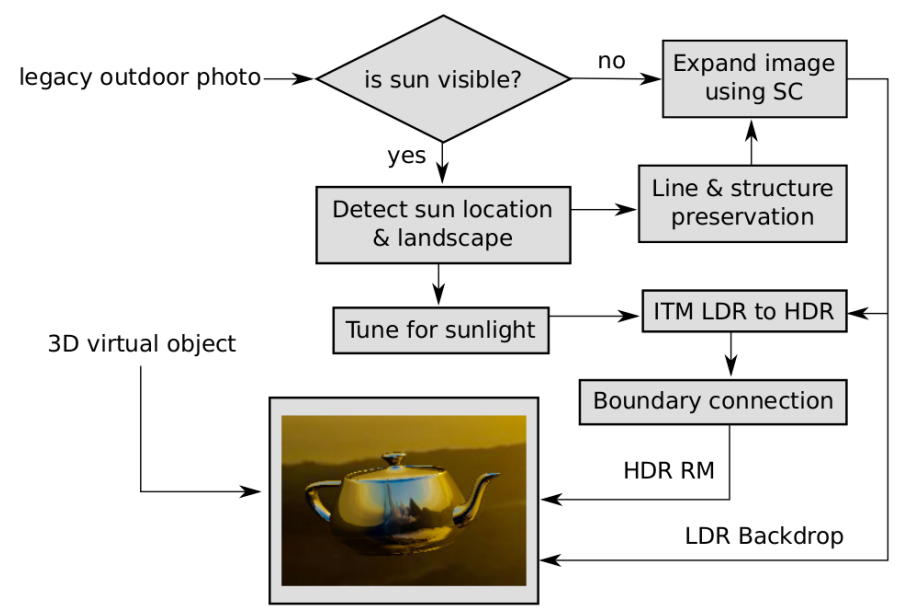

$\mathrm{BBL}$ result

Figure 5.6: Pipeline for IBL using legacy photographs. Here SC means seam carving, ITM inverse tonemapping, and RM radiance map. Diagram from [33].

Kurt Ma, Thomas Iorns, Andrew Chalmers and Taehyun Rhee [33]. In particular work relating to seam carving was carried out by the primary author, Kurt Ma. My contribution was that of providing real-time IBL using the generated image, and developing the real-time HMD-based viewing interface. 


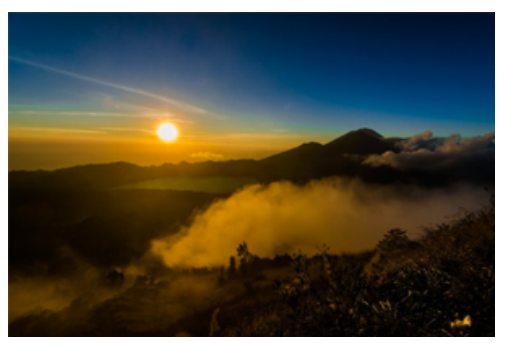

(a) Original photograph.

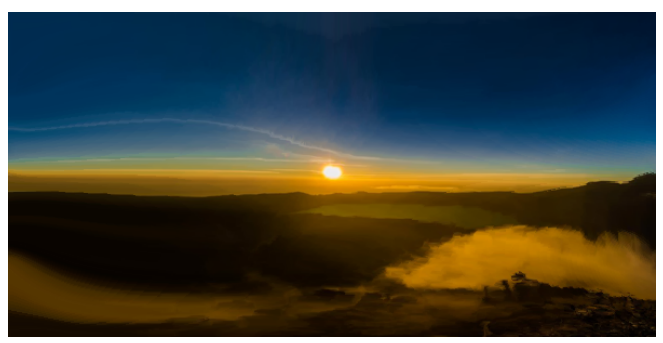

(b) Photograph expanded with seam carving. In this case the sun was deliberately centered.

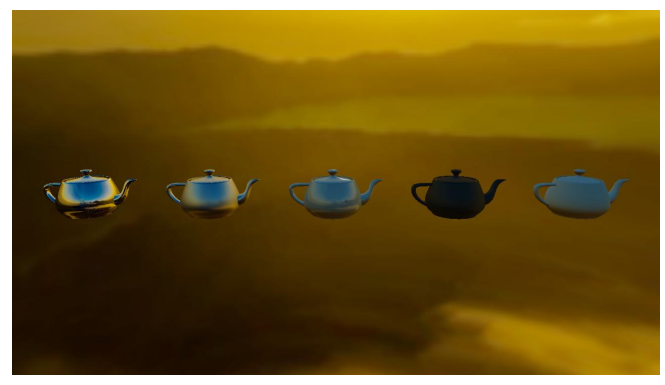

(d) A perspective view of the scene using the expanded photo as the environment. Objects are lit using IBL based on this seamcarved environment.

Figure 5.7: Application of seam carving to provide an environment map for use with IBL (right) compared with simply stretching the original photo (left). 


\section{Chapter 6}

\section{Self-Shadowing and Self-Reflection}

An important aspect of real-time lighting that has not been considered in the IBL implementations put forward so far, is that of shadowing and reflection. Shadows and reflections from the real world onto a virtual object are handled automatically by the application of IBL, but shadows and reflections from the object onto the scene are not considered, neither are shadows and reflections between various virtual objects nor shadows and reflections of a single object onto itself.

When using spherical harmonics for diffuse IBL it has been shown that realtime soft-shadowing is possible [43], however this does not take into account hard shadows or reflections. Here the desire is to develop a potentially novel extention to the previously described system showing that real-time inter-object and intra-object self-shadowing and reflection may be possible alongside the previously developed techniques. As a first step towards this, the problem of self-shadowing and selfreflection when using these real-time IBL techniques is considered, and will form the primary focus of this chapter.

\section{Problem Description}

Develop a system for determining self-occlusion and self-reflection that can be run in real-time and combined with the sampling methods used in the real-time IBL system from chapter 4 . Ideally the result should be usable both in the case of a single virtual 
object and in the case of multiple virtual objects.

Object-scene shadowing and reflection will not be considered, as it requires a virtual reproduction of the environment to be constructed or obtained, which is considered out of scope for this problem. If such a scene is available, similar methods to those of inter- and intra-object shadowing and reflection should be applicable.

\section{Background}

\section{Self-Shadowing}

Many methods for self-shadowing exist, among which the most prominent are ambient occlusion [55] and precompted radiance transfer [45], both commonly used for soft shadowing effects. They require that object geometry be static, or statically animated such that the exact geometry is known in all cases.

Ambient occlusion (see figure 6.1a) is fairly straightforward to implement but leads to unrealistic results. At each vertex of a static object the amount of the outward-facing hemisphere around that vertex which is occluded by the object itself is precomputed. These proportions are extrapolated and used to directly decrease the value of outbound radiance computed for a surface point when doing lighting calculations. This results in soft shadows inside creases and crevices of the object, which gives a generally pleasing appearance. However as it does not take into account the actual direction of lighting in the scene, it cannot provide a realistic approximation of physically accurate shadowing.

Precomputed radiance transfer (see figure 6.1b) uses a similar but more accurate technique. Instead of a simple scalar representing the proportion of the hemisphere which is occluded at each point, occlusion is stored in some directional form. A common application method where soft shadowing is desired is to use spherical harmonics to represent hemispherical occlusion at each vertex of the object, but other systems can be employed. This directional model of occlusion can be used to much more accurately determine the appropriate weighting of light sources in traditional rendering, or irradiance map samples in a sampling-based IBL system such as that 
from chapter 4. The main drawback is that as the occlusion information is stored per vertex, improvement in quality results in a rapid increase in information stored. Typically a vertex on a 3D model will have six to twelve values associated with it (three for position, three for surface normal, and perhaps some more for additional information), so exceeding this number of extra values to obtain a high quality occlusion map at each vertex quickly increases the size of the data structure required.

Although ambient occlusion is not suitable for self-reflection, precomputed radiance transfer can be if information about the occluding surface in a given direction is retained. For this reason it was decided to attempt to find a similar solution that would be applicable to the real-time IBL techniques developed in this thesis.

\section{Coherent Shadow Maps}

One interesting alternate solution for self-occlusion is that of coherent shadow maps described by Ritschel et al. [44]. In this technique a number of orthographic depth images (see figure 6.2) of the model are created. These represent orthographic projections of the object from various angles, with pixel intensity corresponding to depth in the direction of projection.

Given this set of orthographic projections, occlusion can be tested for by taking the orthographic projection most closely matching with the direction of incoming light, and comparing the depth of the current surface point with that of the appropriate pixel in this orthographic depth image. If the surface point in question is significantly behind the position represented by the value in the depth image then it is assumed to be occluded in this direction. Thus occlusion testing is done with a single lookup into a set of precomputed textures. The result is effectively similar to that of precomputed radiance transfer, except using a single set of orthographic depth images for the entire object instead of storing occlusion at each vertex.

By exploiting the coherence of nearby projections a large number of orthographic depth images can be combined, resulting in the data structure referred to as a coherent shadow map. By making certain assumptions about the intended usage of the depth images, a high ratio of compression is able to be obtained meaning that a large number of depth images (typically tens of thousands) can be represented 


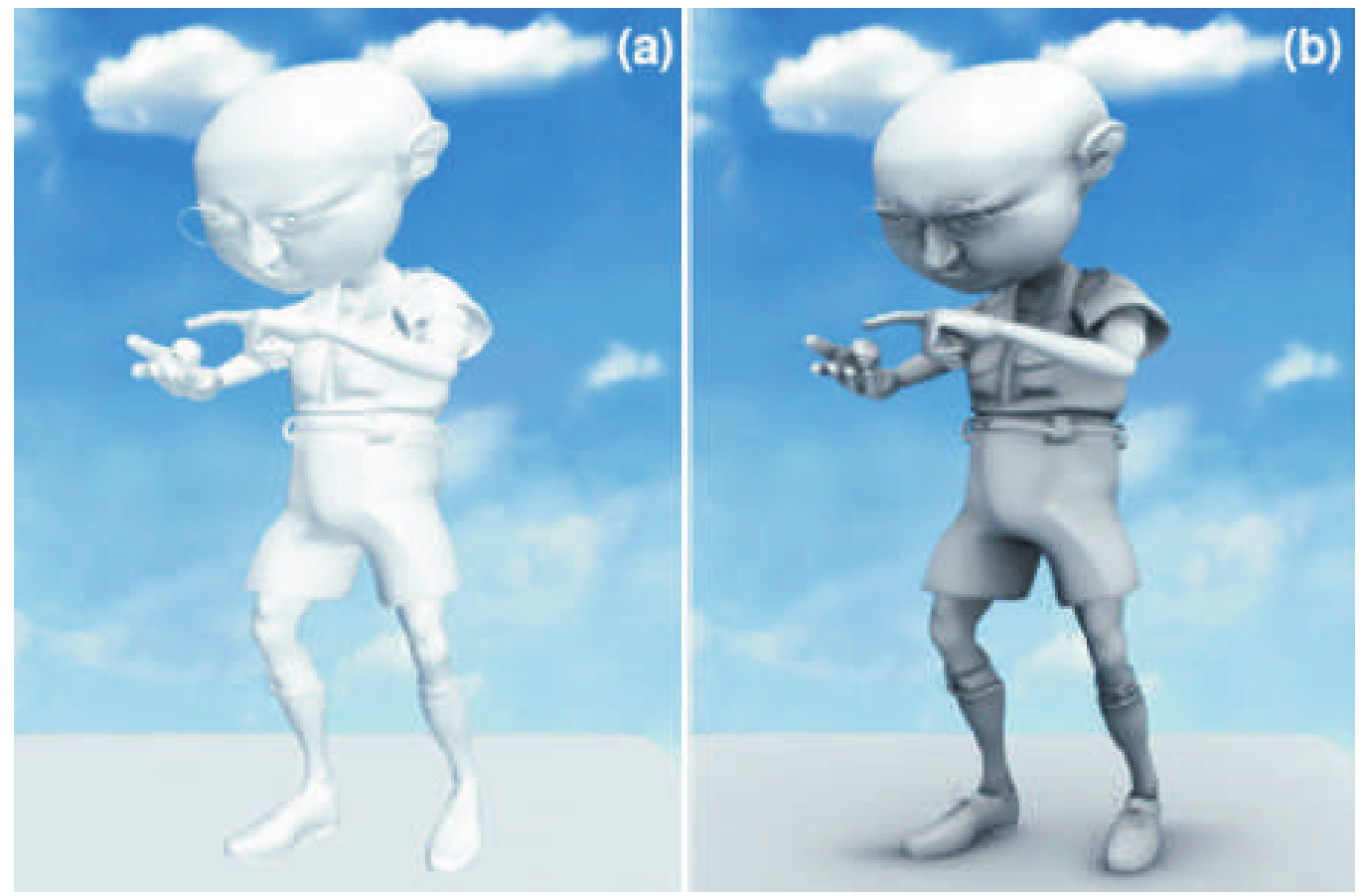

(a) Without (left) and with (right) ambient occlusion. Image from [38].
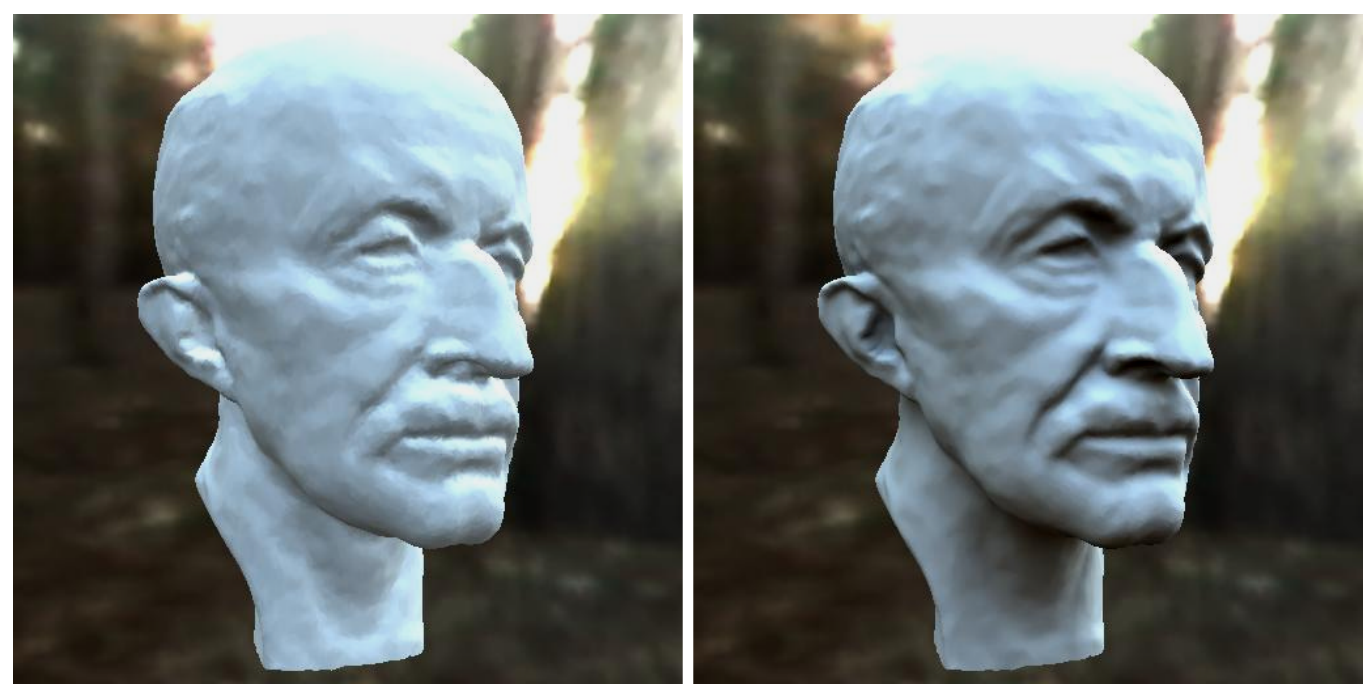

(b) Without (left) and with (right) precomputed radiance transfer. Image from [45].

Figure 6.1: Examples of ambient occlusion and precomputed radiance transfer. 

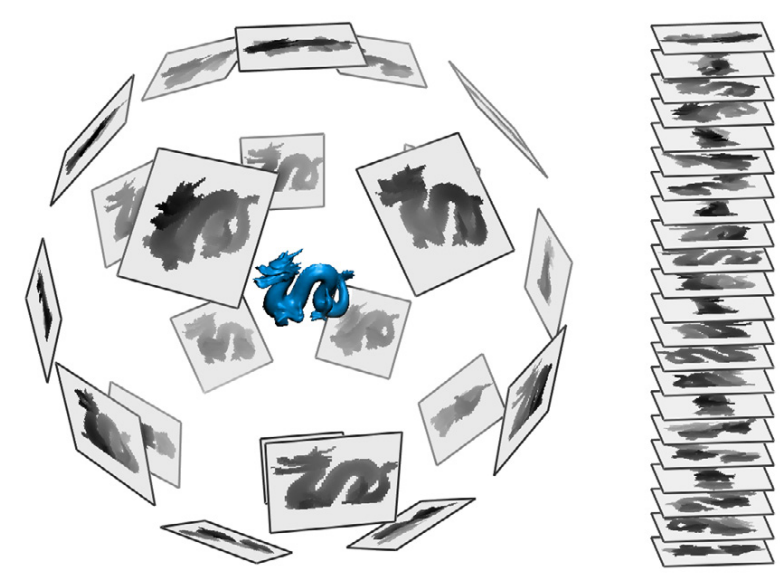

Figure 6.2: Coherent Shadow maps. Depth images are taken from a variety of angles, and these are compressed into a combined data structure. Image from [44].

relatively cheaply.

Unfortunately because of both the visibility testing method employed and the compression technique used coherent shadow maps are not suitable for reflection computations and are limited to occlusion. As a benefit however, testing for interobject occlusion can be done using the exact same process as intra-object occlusion by treating the point in question as if it were part of the other object. As such this technique is suitable for both self-shadowing and mutual shadowing between objects.

\section{Layered Depth Images}

An alternate method of real-time occlusion testing is presented by Nießner et al. [36], in which they use orthographic layered depth images of the entire scene instead of plain depth images of individual objects.

Layered depth images are similar to the orthographic depth images described in section 6.2.2, however instead of simply storing the nearest depth value at each pixel, depth values for all surface intersections are stored. This can be done using a fixed amount of memory to assign a fixed number of layers, or it can be done using a compressed data structure to store a variable number of layers per pixel. In [36] a 


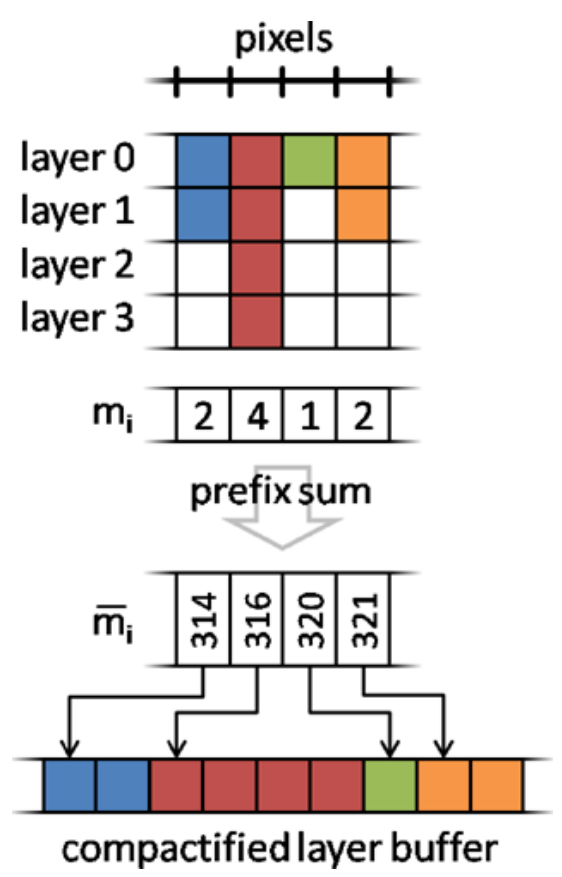

Figure 6.3: Layered Depth Image stored in a one-dimensional data structure. Number of layers are counted at each pixel, and this number is used to concatenate layer information at the same time as indexing it by pixel. Image from [36].

one-dimensional data structure is used to store the depth values for an entire image, and an indexing image stores offsets into this one-dimensional data structure (see figure 6.3). If values are stored in order, the number of layers at a given index pixel can be obtained by comparing the value of the offset at the following pixel.

So as to compensate for the low number of projections compared to that of coherent shadow maps, instead of a single lookup a ray-marching technique is used to test for occlusion (see figure 6.4). According to the size of the scene and the direction of the ray representing an occlusion test, a small number of pixels are tested in the layered depth image most closely corresponding to the direction of the occlusion test. If the occlusion test is in exactly the direction of the depth image only one pixel needs to be checked, but if the angle between occlusion ray and depth image direction is large several pixels may have to be traversed to determine whether or not there is an occlusion before arriving at the surface point which generated the query. 


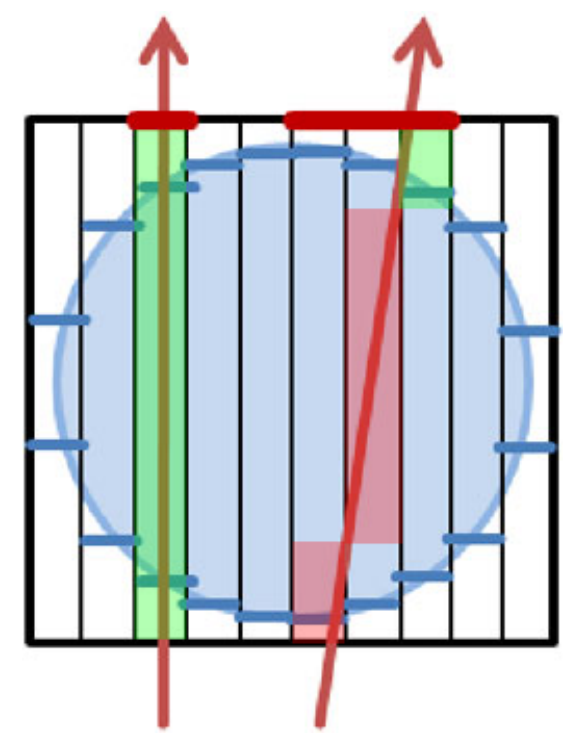

Figure 6.4: Ray marching using layered depth image. Surface intersections are tested for at each traversed pixel according to the depth region in which the ray intersects that pixel. Projection direction here is oriented vertically, with each pixel containing two layers representing the front and back surface of the circle. If the ray is parallel to the projection direction (left) only one pixel is tested, but several may need to be tested (right) if the relative angle is large. For exposition the back surface is being tested for here, but the algorithm would usually terminate at the front surface. Image adapted from [36].

The complexity of this operation thus depends on the number of depth images provided, decreasing as this number increases and thus the maximum angle between images grows smaller. As the entire scene is captured, the occlusion test has constant complexity with respect to the number of objects, making it potentially more suitable than coherent shadow maps for scenes with multiple objects.

The primary drawback however is that it only works for static scenes. Each depth image requires a rasterization of the entire scene, and it is impractical to do this in real-time on current hardware. Dynamic objects can be included in the scene, and will take into account shadows and reflections from the static scene, but the dynamic object itself will not be accounted for in scene illumination and shadowing. 
Another drawback relates to the amount of memory necessary to store the depth images. Although layers are compressed, it does not come near the compression ratios achievable using coherent shadow maps. Thus the higher the desired quality and the lower the desired real-time computation burden, the more memory must be used to store the layered depth images, and the more precompution time must be spent generating and compressing them.

One important benefit of this technique over coherent shadow maps, however, is that the compressed layered depth images are not limited to storing only depth data. If other surface information such as surface normal direction is also stored then it becomes possible to use this technique for reflection, and potentially other purposes.

\section{Orthographic Linearized Layered Fragment Buffers (OLLFBs)}

The similarities between the techniques of subsections 6.2.2 and 6.2.3 as well as their complementary advantages and disadvantages suggest that a similar technique might be suitable for real-time self-shadowing and self-reflection in the real-time IBL framework of chapter 4 . If the ability of the layered depth images technique to handle reflection and indirect illumination could be combined with the ability of the coherent shadow maps technique to handle dynamic scenes, it could provide a suitable solution for use in real-time IBL. As such, a potentially novel system is developed extending [44] and [36] to apply self-shadowing and self-reflection to individual objects in real-time, and its usage in the previously described real-time IBL framework is examined.

\section{Implementation}

As the initial concern is for self-shadowing and self-reflection on a single object, this single object can be considered as an entire scene and the technique of section 6.2.3 directly implemented utilizing layered depth images. For the implementation here the method described by Knowles et al. [26] is followed, and the terminology 
from this source will also be used, referring to the linear data structures holding depth information as linearized layered fragment buffers. Following this convention the orthographic projections using them will be referred to here as orthographic linearized layered fragment buffers (OLLFBs).

To construct a single OLLFB an orthographic projection of the 3D model in question is rasterized in a two-pass procedure. The first pass counts the number of fragments at each pixel, creating an image where each pixel contains an integer representing this layer count. This can be done efficiently in OpenGL using the atomicAdd functionality available from version 4.3. From this image containing fragment counts another image is constructed containing what will eventually be the offsets into the final linearized fragment buffer.

In the second rasterization pass, fragment depth and normal direction are stored in the fragment buffer according to pixel offset. The previous counter image is reused to ensure that each fragment is stored at a unique location. After this pass the fragments at each pixel position in the buffer are sorted by depth for more convenient access, and the OLLFB is considered to have been constructed for this particular projection.

Repeating the procedure for several directions results in a set of OLLFBs. The exact number can be manipulated to trade memory usage for occlusion test speed. Once the full set of OLLFBs has been generated, occlusion tests can be done in a manner similar to that of [36], ray marching across pixels in the single most appropriate OLLFB.

\section{Results}

The functionality of the prototype used in section 4.1 was extended to generate OLLFBs as described in section 6.3.1, and their use in IBL was then tested. As using similar structures for shadowing has already been explored in [44] and [36], in this case only self-reflection was examined. As the occlusion test used in shadowing is also part of any reflection test, self-shadowing performance is expected to be equivalent to or better than that of self-reflection, with results similar to those of 

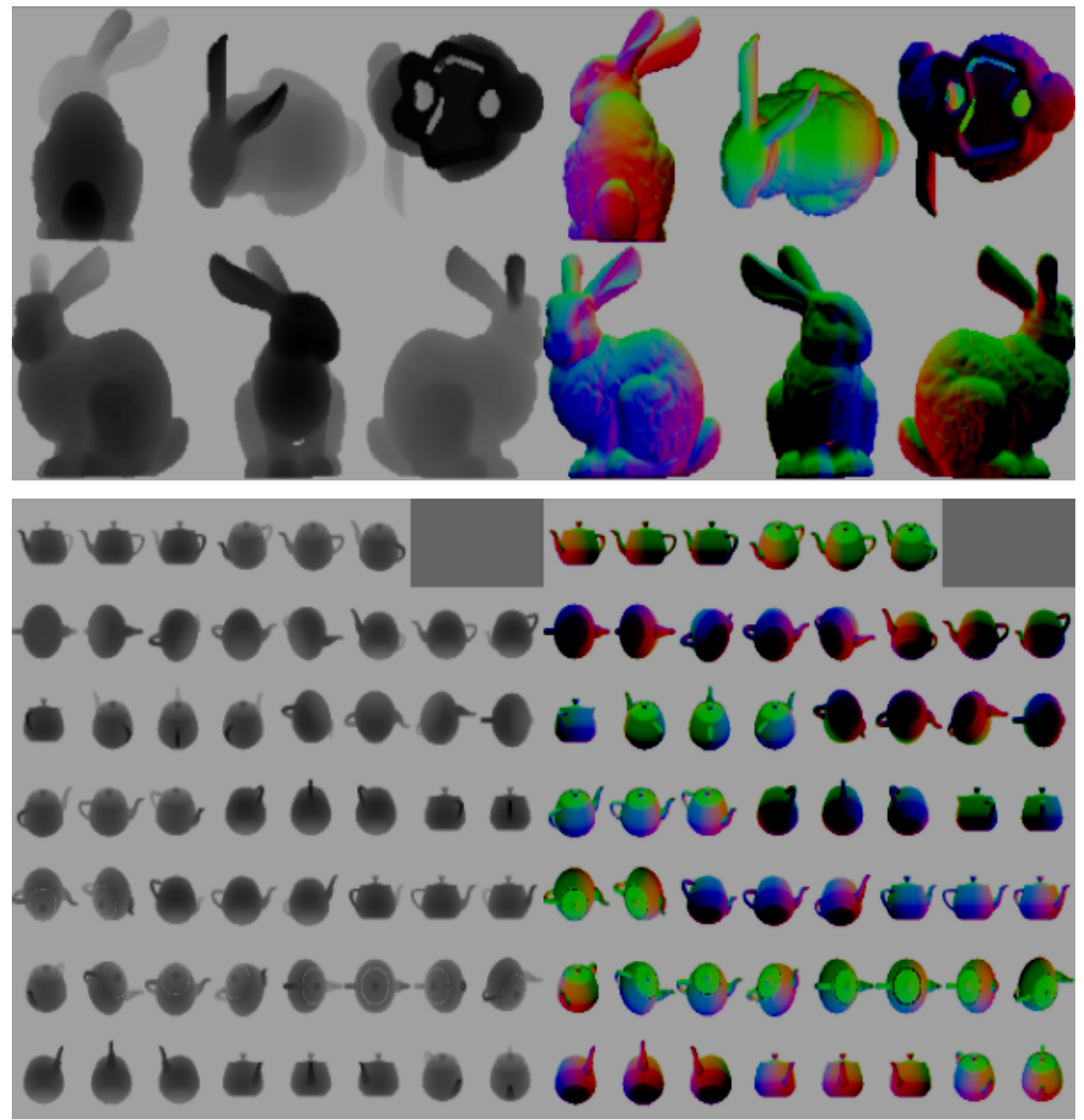

Figure 6.5: A visualization of OLLFB data for six directions of a bunny model (top) and 54 directions of a teapot model (bottom). The greyscale images (left) represent depth, while the colour images (right) represent surface normal direction. Only the topmost layer is visualized here, but all layers are stored. 


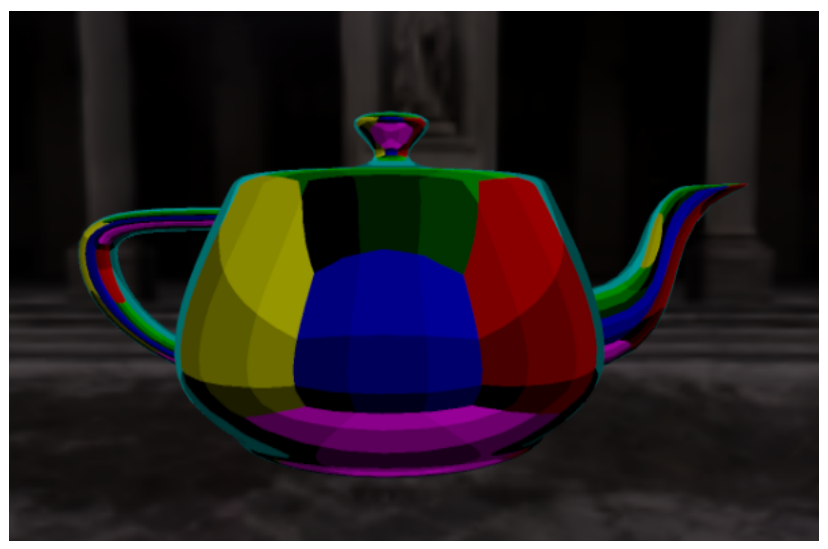

Figure 6.6: A direct depiction of which OLLFB is queried when rendering each point on a virtual teapot. Here OLLFBs are divided into six groups of nine, each group corresponding to an axial direction in the model's local coordinate system. Colour corresponds to group, while shading corresponds to individual OLLFB. The OLLFB data that would be used for rendering in this case can be seen in figure 6.5.

previous work.

The occlusion test is implemented in the same way for reflection and shadowing, by taking the surface point performing the test and the sample direction to be tested, and converting them into the frame of reference of the most appropriate OLLFB (see figure 6.6). Instead of performing the occlusion test from the outside as in [44, 36], it is performed in the direction of the sample test. While this allows the closest occluding layer in the sampling direction to be determined, it can take much longer than testing in reverse as multiple layers may have to be tested before the correct layer is found.

The simplest tested implementation making use of this procedure was that of single-bounce pure specular self-reflection. In this case only a single sample ray needs be tested for possible self-reflection. Starting with the direction of specular reflection that would normally be sampled from in pure specular IBL, an occlusion test is performed using the OLLFBs, as described in section 6.2.3. If an occlusion is found, the surface normal direction corresponding to this occlusion is determined from the OLLFB and the sampling direction is reflected across it, thus performing 
another iteration of pure specular reflection. This final doubly-reflected direction is then used as the sampling direction for IBL.

Results for this can be seen in figure 6.7. Here 486 OLLFBs of $64 \times 64$ resolution were used, requiring $26 \mathrm{MiB}$ of graphics memory for storage. Output framerate was approximately halved compared to that without self-reflection. Although a clear difference is visible in the results with and without self-reflection, preliminary subjective observation was that the biggest improvement in the quality of the results when self-reflection was enabled related to changes in the surface reflection as the object or view was moved, an effect that is difficult to display here.

A similar self-reflection method was subsequently applied to glossy specular reflection (see figure 6.8). As in chapter 4, 36 sample rays per pixel were used to simulate glossy reflection, and a self-reflection test was done for each one individually. In this case instead of repeating the glossy sampling procedure to determine the reflected colour, a simple pure specular lookup was performed. The result was able to run at over $60 \mathrm{~Hz}$ on the Oculus Rift DK2 with its default resolution of $1182 \times 1464$ using an Nvidia GeForce 980.

The effect of varying both number and resolution of OLLFBs was examined, and as expected it was found that reducing the number of OLLFBs increased reflection computation time significantly. Contrarily reducing the resolution of OLLFBs instead reduced reflection computation time, but resulted in significant visible pixelization artifacts (see figure 6.10) as resolution was decreased. This can be attributed to the naïve sampling method implemented here, which only takes the depth and surface normal values of the nearest OLLFB pixel, and does not attempt to extrapolate or interpolate between pixels. Such interpolation would likely significantly improve results.

There are also occasional gaps in the reflection, caused by the crude treatment of surfaces when performing the ray-marching algorithm across the OLLFB. This problem is as described in [36] and depicted in figure 6.9. As the current implementation differs from [36] with the addition of surface normal information, an improved implementation taking into account surface slope should be possible, but this was not attempted here. 

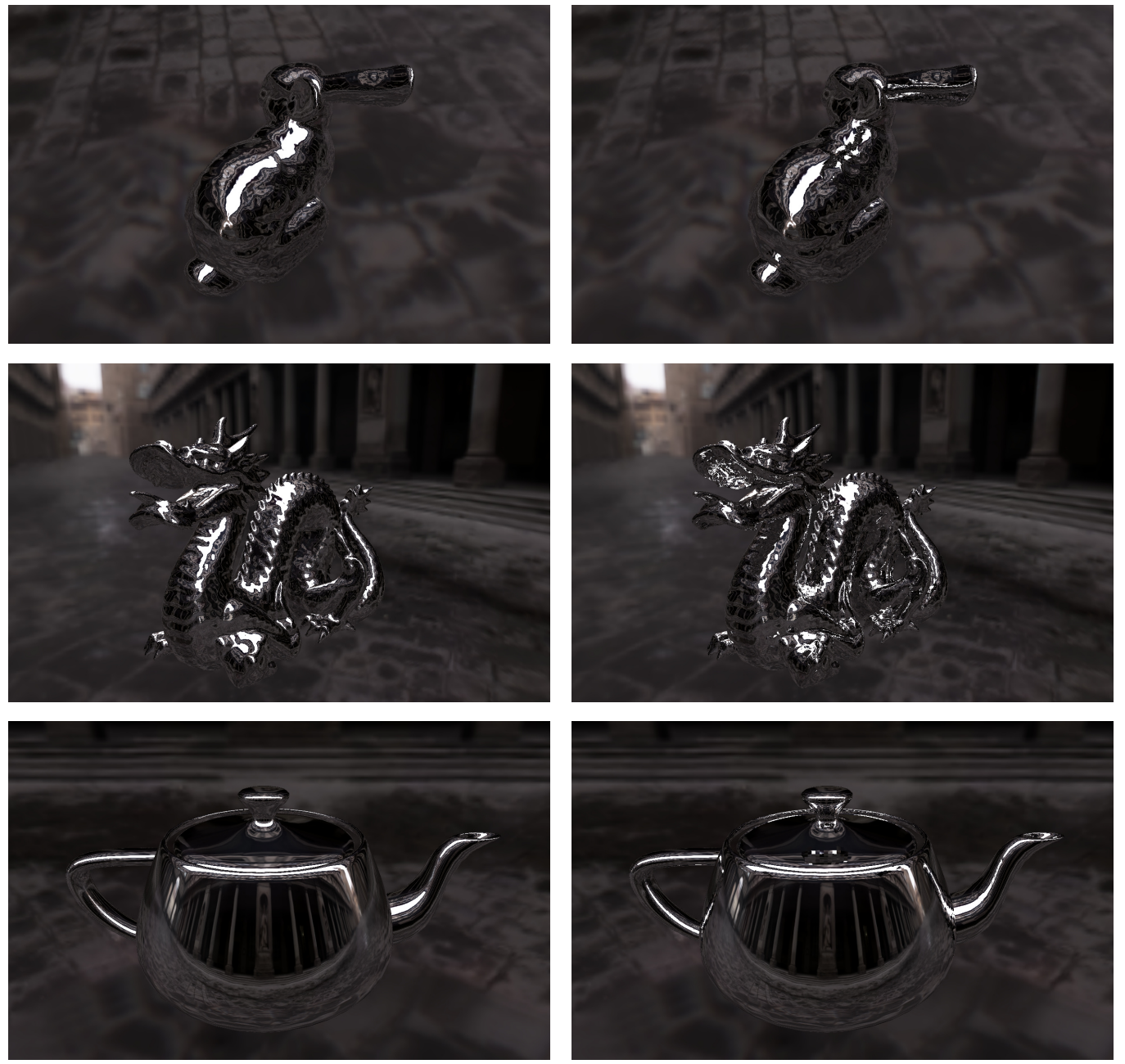

Figure 6.7: Results with and without single-bounce self-reflection using OLLFBs. Images on the left are rendered using pure specular IBL, with those on the right including single-bounce specular self-reflection. The difference is subtle, but distinctly noticable in cases such as the bunny's ears, the dragon's mouth, and the teapot's spout, handle and lid. 

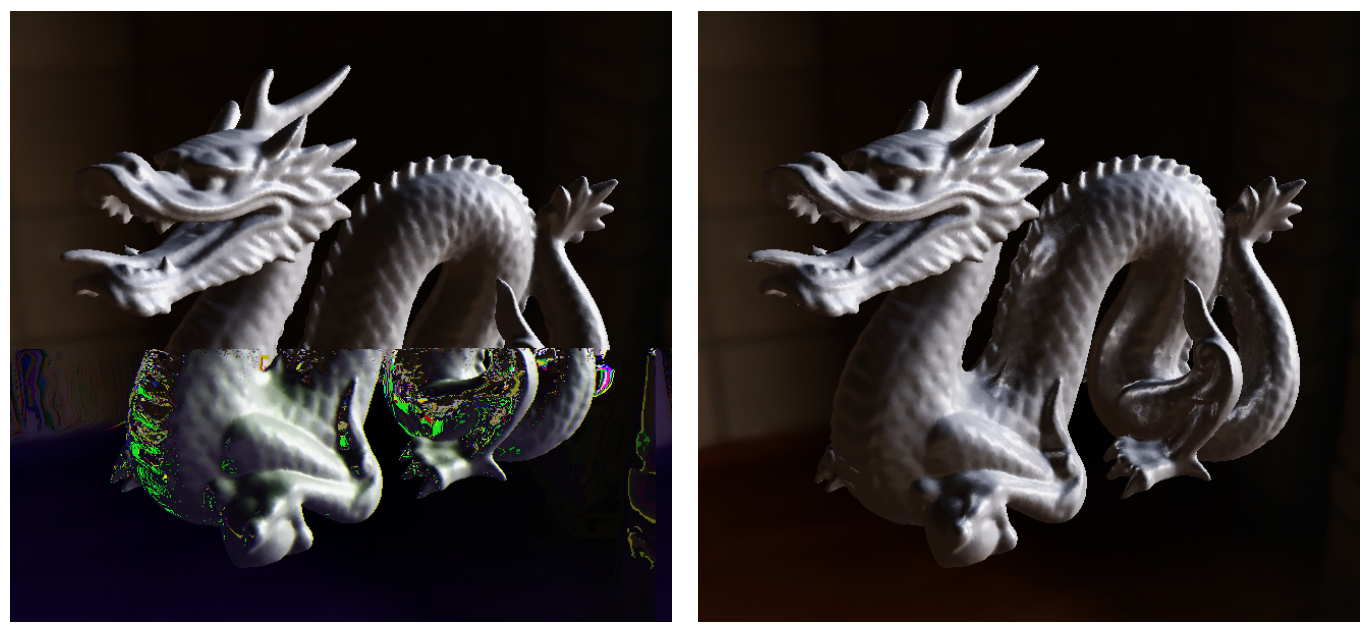

Figure 6.8: Glossy specular reflection with single-bounce self-reflection.
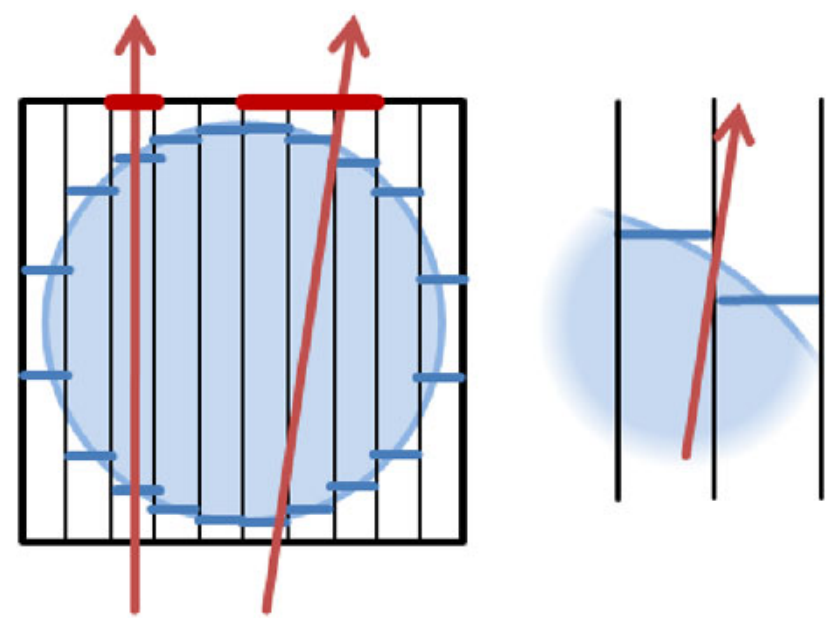

Figure 6.9: If a cast ray almost reaches a layer but then reaches the edge of the pixel being considered, it is possible to miss the surface when using the naïve method depicted here of simply comparing the stored depth value of the layer to the point at which the ray leaves the pixel volume. Image from [36]. 


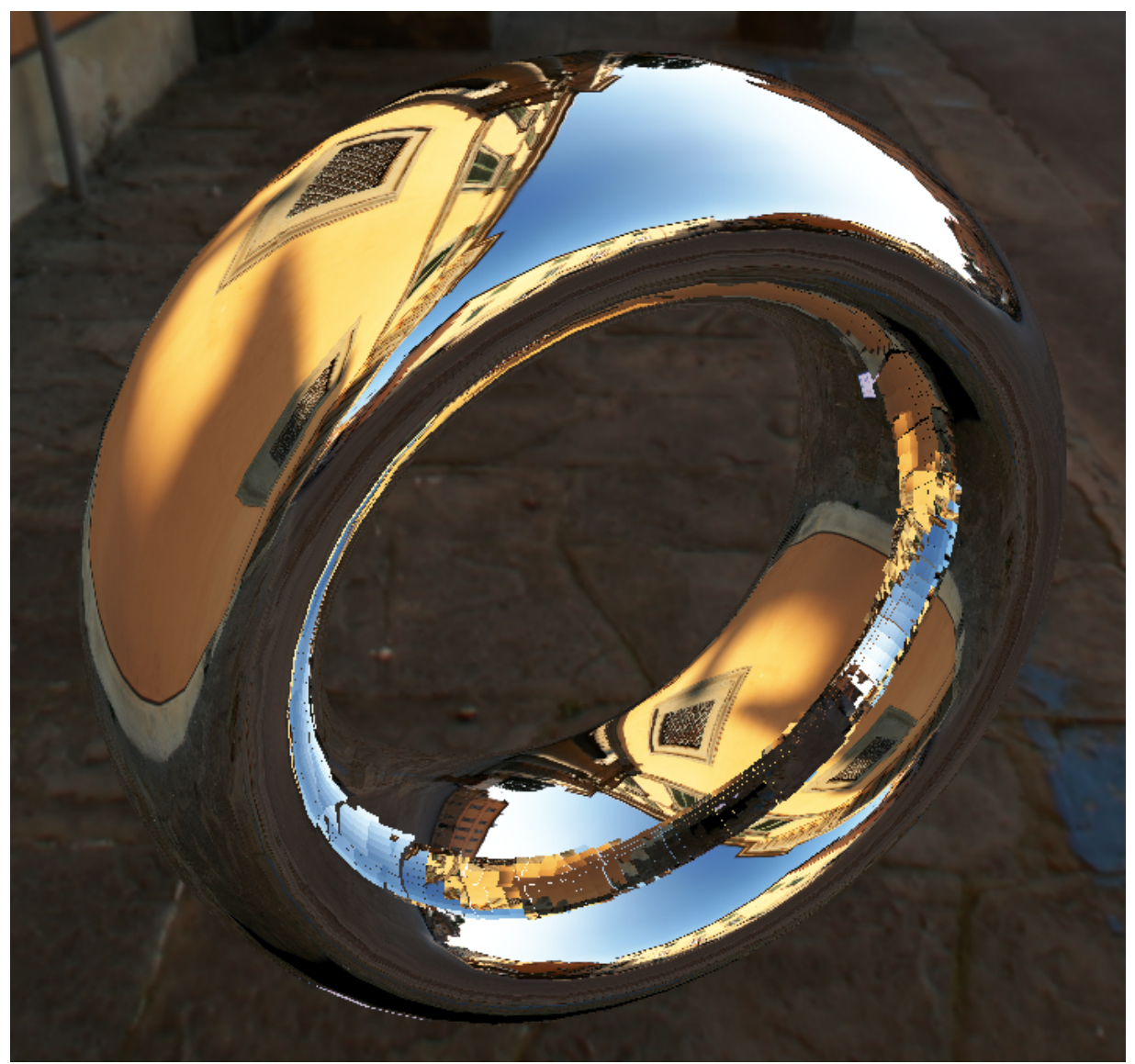

Figure 6.10: A shiny torus showing some of the problems that can occur with singlebounce reflection using low resolution OLLFBs. The reflection is pixellated, has visible gaps, and appears strange where the inside of the torus should reflect multiple times but doesn't. Here 150 OLLFBs were used, each of 32x32 resolution, taking up around $3 \mathrm{MiB}$ of memory in total. In this case higher resolution OLLFBs, better ray marching technique, and more iterations of self-reflection would all improve the result. 
On the whole results are promising. With high resolutions and numbers of OLLFBs the reflections are quite accurate, and it is likely that quality can be significantly improved by addressing the pixelization and disjointedness problems mentioned above.

From the above tests it seems possible that believable real-time self-shadowing and self-reflection may be achievable using this technique. Real-time self-reflection has been successfully added as an extension to the real-time IBL technique developed in chapter 4 , and this has shown promising results. In addition there is little preventing this technique from being applied to self-shadowing, and even multiobject shadowing and reflection, so long as the number of objects being tested for occlusion is kept to a minimum.

Although several problems exist with the basic implementation described here, higher quality results should be obtainable by improving the occlusion-testing algorithm used, and two plausible methods of doing so have been proposed.

On the whole the basic success of this technique demonstrates the possibility that the previously developed real-time IBL technique can be effectively extended to include real-time inter- and intra-object shadowing and reflection. 


\section{Chapter 7}

\section{Conclusion}

In this thesis a system was proposed for the application of image based lighting in real-time using 360 degree panoramic video as an environment map. The proposed system was shown to work effectively, rendering believable virtual objects using standard low dynamic range video as input, with no precomputation or prior analysis of the video required. No guidance was necessary at any stage of the process, and the same implementation parameters were used for all tested input cases. The result was shown to render at the high resolutions and framerates necessary for comfortable and immersive viewing on stereographic head-mounted display.

The system was then applied to several real-world scenarios, and found to perform well in each of them. Implementation process was clear even when converting between frameworks, and the primary benefits of the approach detailed in this thesis, applicability to both MR applications and standard LDR content, were demonstrated.

The possibility of extending the system beyond the original scope of the thesis was then explored, constructing and examining a potential system for the incorporation of self-shadowing and self-reflection into the developed process. Results of this brief exploration were promising, with clear directions available for further improvement.

The basic implementation of the real-time IBL system solution also had various aspects that could potentially be improved. Several system components were found 
to be sufficient for use in the final pipeline, but require more research to determine whether or not better components exist. Alternate sampling patterns to the one used for glossy specular reflection were not evaluated, nor were potential filtering techniques other than mipmapping, nor interpolation techniques other than hardware bilinear and trilinear filtering. The inverse tonemapping operator used was found to be effective and robust, but it was not determined precisely why this should be, nor whether the use of another operator might be better.

The primary direction of research that would most improve the current result, however, is probably that of more appropriately matching the rendered virtual objects with the input video environment as it is displayed. Not only were mismatches in resolution evident, but also mismatches due to the nonlinear mapping from rectangular input video frame to spherical environment, which was not reproduced in the perspective rendering of the objects. Emplacing the rendered virtual objects directly into the background video frame somehow would likely greatly improve the seamlessness, and thus perceived realism, of the result.

Overall the solution presented in this thesis has been shown to be effective, efficient and extensible, building and improving on previous work in the field to utilize the newly emergant content medium of 360 degree panoramic video, and leading into several potential areas of continuing research. 


\section{Bibliography}

[1] Sameer Agarwal, Ravi Ramamoorthi, Serge Belongie, and Henrik Wann Jensen. "Structured importance sampling of environment maps". In: ACM Transactions on Graphics (TOG) 22.3 (2003), pp. 605-612.

[2] Kusuma Agusanto, Li Li, Zhu Chuangui, and Ng Wan Sing. "Photorealistic rendering for augmented reality using environment illumination”. In: Mixed and Augmented Reality, 2003. Proceedings. The Second IEEE and ACM International Symposium on. IEEE. 2003, pp. 208-216.

[3] Ahmet Oğuz Akyüz, Roland Fleming, Bernhard E Riecke, Erik Reinhard, and Heinrich H Bülthoff. "Do HDR displays support LDR content?: a psychophysical evaluation”. In: ACM Transactions on Graphics (TOG). Vol. 26. 3. ACM. 2007, p. 38.

[4] Matthew Anderson, Ricardo Motta, Srinivasan Chandrasekar, and Michael Stokes. "Proposal for a standard default color space for the internet-srgb". In: Color and imaging conference. Vol. 1996. 1. Society for Imaging Science and Technology. 1996, pp. 238-245.

[5] Shai Avidan and Ariel Shamir. "Seam carving for content-aware image resizing”. In: ACM Transactions on graphics (TOG). Vol. 26. 3. ACM. 2007, p. 10.

[6] James F Blinn and Martin E Newell. "Texture and reflection in computer generated images". In: Communications of the ACM 19.10 (1976), pp. 542547. 
[7] R Bogart, F Kainz, and D Hess. "OpenEXR image file format". In: ACM SIGGRAPH 2003, Sketches \& Applications (2003).

[8] David Burke, Abhijeet Ghosh, and Wolfgang Heidrich. "Bidirectional Importance Sampling for Direct Illumination.” In: Rendering Techniques 5 (2005), pp. 147-156.

[9] Andrew Chalmers, Jong Jin Choi, and Taehyun Rhee. "Perceptually Optimised Illumination for Seamless Composites”. In: Pacific Graphics Short Papers. Ed. by John Keyser, Young J. Kim, and Peter Wonka. The Eurographics Association, 2014. ISBN: 978-3-905674-73-6. DoI: 10. 2312/pgs . 20141268.

[10] Paul Debevec. "Image-based lighting". In: IEEE Computer Graphics and Applications 2 (2002), pp. 26-34.

[11] Paul Debevec. "Rendering synthetic objects into real scenes: Bridging traditional and image-based graphics with global illumination and high dynamic range photography”. In: ACM SIGGRAPH 2008 classes. ACM. 2008, p. 32.

[12] Raanan Fattal, Dani Lischinski, and Michael Werman. "Gradient domain high dynamic range compression". In: ACM Transactions on Graphics (TOG). Vol. 21. 3. ACM. 2002, pp. 249-256.

[13] FreeImage. The FreeImage Project. http : / / freeimage . sourceforge . net/. Accessed: 2016-03-20. 2015.

[14] Ned Greene. "Applications of world projections". In: Proceedings of Graphics Interface'86. 1986, pp. 108-114.

[15] $\mathrm{HDI}^{2}$ 4D. Human-Digital Content Interaction for Immersive 4D Home Entertainment. http: // computergraphics . ac .nz/hdi4d/. Accessed: 201603-20. 2016.

[16] Wolfgang Heidrich and Hans-Peter Seidel. "Realistic, hardware-accelerated shading and lighting”. In: Proceedings of the 26th annual conference on Computer graphics and interactive techniques. ACM Press/Addison-Wesley Publishing Co. 1999, pp. 171-178. 
[17] holmen. Fish Perch. http://www. blendswap.com/blends/view/67777. Accessed: 2016-03-20. 2013.

[18] David Hsieh. Scuba Diving Short Film in $360^{\circ}$ Green Island, Taiwan. http: / / www . youtube . com / watch ? v=20zlksZBTiA. Accessed: 2016-03-20. 2015.

[19] GoPro Inc. GoPro Official Website. http://gopro.com/. Accessed: 201603-20. 2016.

[20] Leap Motion Inc. Leap Motion. http : //www . leapmotion. com/. Accessed: 2016-03-20. 2016.

[21] Thomas Iorns and Taehyun Rhee. "Real-Time Image Based Lighting for 360 Degree Panoramic Video". In: Lecture Note in Computer Science. Presented in PSIVT workshop, Vision Meets Graphics 2015, Auckland, NZ, Nov, 2015. Springer, 2015.

[22] Kevin Karsch, Varsha Hedau, David Forsyth, and Derek Hoiem. "Rendering synthetic objects into legacy photographs". In: ACM Transactions on Graphics (TOG). Vol. 30. 6. ACM. 2011, p. 157.

[23] Kevin Karsch, Kalyan Sunkavalli, Sunil Hadap, Nathan Carr, Hailin Jin, Rafael Fonte, Michael Sittig, and David Forsyth. "Automatic scene inference for 3d object compositing”. In: ACM Transactions on Graphics (TOG) 33.3 (2014), p. 32 .

[24] Jan Kautz and Michael D McCool. "Approximation of glossy reflection with prefiltered environment maps". In: Graphics Interface. Vol. 2000. 2000, pp. 119126.

[25] Gary King. "Real-time computation of dynamic irradiance environment maps". In: GPU Gems 2 (2005), pp. 167-176.

[26] Pyarelal Knowles, Geoff Leach, and Fabio Zambetta. “OpenGL Insights”. In: ed. by Patrick Cozzi and Christophe Riccio. CRC press, 2012. Chap. 20, pp. 279-292. 
[27] Eugenia M Kolasinski. Simulator Sickness in Virtual Environments. Tech. rep. DTIC Document, 1995.

[28] Jaroslav Křivánek and Mark Colbert. "Real-time Shading with Filtered Importance Sampling”. In: Computer Graphics Forum. Vol. 27. 4. Wiley Online Library. 2008, pp. 1147-1154.

[29] Joel Kronander, Johan Dahlin, Daniel Jonsson, Manon Kok, Thomas Schon, and Jonas Unger. "Real-time video based lighting using GPU raytracing". In: Signal Processing Conference (EUSIPCO), 2014 Proceedings of the 22nd European. IEEE. 2014, pp. 1627-1631.

[30] Hayden Landis. "Production-ready global illumination". In: Siggraph course notes 16.2002 (2002), p. 11.

[31] Blend Swap LLC. Blend Swap. http: //blendswap. com. Accessed: 201603-20. 2016.

[32] Mark Loftesnes. Great White Shark. http : / / www . blendswap . com / blends/view/80243. Acessed: 2016-03-20. 2015.

[33] Wan Duo Ma, Thomas Iorns, Andrew Chalmers, and Taehyun Rhee. "Synthesizing Radiance Maps from Legacy Outdoor Photographs for Real-time IBL on HMDs". In: Proc. of 30th International Conference on Image and Vision Computing New Zealand (IVCNZ 2015). IEEE, 2015.

[34] Nick Michiels, Lode Jorissen, Jeroen Put, and Philippe Bekaert. "Interactive Augmented Omnidirectional Video with Realistic Lighting”. In: Augmented and Virtual Reality. Springer, 2014, pp. 247-263.

[35] Gene S Miller and C Robert Hoffman. "Illumination and reflection maps: Simulated objects in simulated and real environments". In: SIGGRAPH 84 Advanced Computer Graphics Animation seminar notes. Vol. 190. 1984.

[36] Matthias Nießner, Henry Schäfer, and Marc Stamminger. "Fast indirect illumination using layered depth images”. In: The Visual Computer 26.6-8 (2010), pp. 679-686.

[37] OpenCV. OpenCV. http: //opencv.org/. Accessed: 2016-03-20. 2015. 
[38] Saulo A Pessoa, Eduardo L Apolinario, Guilherme de S Moura, Joao Paulo S do M Lima, Márcio AS Bueno, Veronica Teichrieb, and Judith Kelner. "Illumination techniques for photorealistic rendering in augmented reality". In: X Symposium on Virtual and Augmented Reality, João Pessoa, PB, Brasil. 2008, pp. 223-232.

[39] Bui Tuong Phong. "Illumination for computer generated pictures". In: Communications of the ACM 18.6 (1975), pp. 311-317.

[40] Hugin project. Hugin Panorama Photo Stitcher. http://hug in. sourceforge. net/. Accessed: 2016-03-20. 2016.

[41] Ravi Ramamoorthi and Pat Hanrahan. "An efficient representation for irradiance environment maps". In: Proceedings of the 28th annual conference on Computer graphics and interactive techniques. ACM. 2001, pp. 497-500.

[42] Erik Reinhard, Michael Stark, Peter Shirley, and James Ferwerda. "Photographic tone reproduction for digital images". In: ACM Transactions on Graphics (TOG). Vol. 21. 3. ACM. 2002, pp. 267-276.

[43] Zhong Ren, Rui Wang, John Snyder, Kun Zhou, Xinguo Liu, Bo Sun, PeterPike Sloan, Hujun Bao, Qunsheng Peng, and Baining Guo. "Real-time soft shadows in dynamic scenes using spherical harmonic exponentiation". In: ACM Transactions on Graphics (TOG) 25.3 (2006), pp. 977-986.

[44] Tobias Ritschel, Thorsten Grosch, Jan Kautz, and Stefan Müeller. "Interactive illumination with coherent shadow maps". In: Proceedings of the 18th Eurographics conference on Rendering Techniques. Eurographics Association. 2007, pp. 61-72.

[45] Peter-Pike Sloan, Jan Kautz, and John Snyder. "Precomputed radiance transfer for real-time rendering in dynamic, low-frequency lighting environments". In: ACM Transactions on Graphics (TOG). Vol. 21. 3. ACM. 2002, pp. 527536.

[46] Assimp Development Team. Open Asset Import Library. http : //assimp . org/. Accessed: 2016-03-20. 2015. 
[47] Unity Technologies. Unity Game Engine. http://unity3d.com. Accessed: 2016-03-20. 2016.

[48] Jonas Unger, Stefan Gustavson, Joel Kronander, Per Larsson, Gerhard Bonnet, and Gunnar Kaiser. "Next generation image based lighting using HDR video”. In: ACM SIGGRAPH 2011 Talks. ACM. 2011, p. 60.

[49] Jonas Unger, Joel Kronander, Peter Larsson, Stefan Gustavson, and Anders Ynnerman. "Temporally and spatially varying image based lighting using HDRvideo". In: Signal Processing Conference (EUSIPCO), 2013 Proceedings of the 21st European. IEEE. 2013, pp. 1-5.

[50] Jonas Unger, Magnus Wrenninge, and Mark Ollila. "Real-time image based lighting in software using HDR panoramas". In: Proceedings of the 1st international conference on Computer graphics and interactive techniques in Australasia and South East Asia. ACM. 2003, 263-ff.

[51] Oculus VR. Oculus Rift. http : / www . oculus . com/en-us /rift. Accessed: 2016-03-20. 2016.

[52] Jiaping Wang, Peiran Ren, Minmin Gong, John Snyder, and Baining Guo. “All-frequency rendering of dynamic, spatially-varying reflectance". In: ACM Transactions on Graphics (TOG). Vol. 28. 5. ACM. 2009, p. 133.

[53] Richard Yao, Tom Heath, Aaron Davies, Tom Forsyth, Nate Mitchell, and Perry Hoberman. “Oculus VR Best Practices Guide”. In: Oculus VR (2014).

[54] YouTube. 360Video. http ://youtube. com/360. Accessed: 2016-03-20. 2016.

[55] Sergey Zhukov, Andrei Iones, and Grigorij Kronin. "An ambient light illumination model”. In: Rendering Techniques' 98. Springer, 1998, pp. 45-55. 